University of Louisville

ThinkIR: The University of Louisville's Institutional Repository

Electronic Theses and Dissertations

$11-2013$

\title{
Cyclin E induction and oncolytic replication of E1b-deleted adenoviruses.
}

Pei-Hsin Cheng

University of Louisville

Follow this and additional works at: https://ir.library.louisville.edu/etd

Part of the Pharmacy and Pharmaceutical Sciences Commons

\section{Recommended Citation}

Cheng, Pei-Hsin, "Cyclin E induction and oncolytic replication of E1b-deleted adenoviruses." (2013). Electronic Theses and Dissertations. Paper 2282.

https://doi.org/10.18297/etd/2282

This Doctoral Dissertation is brought to you for free and open access by ThinkIR: The University of Louisville's Institutional Repository. It has been accepted for inclusion in Electronic Theses and Dissertations by an authorized administrator of ThinkIR: The University of Louisville's Institutional Repository. This title appears here courtesy of the author, who has retained all other copyrights. For more information, please contact thinkir@louisville.edu. 


\title{
CYCLIN E INDUCTION AND ONCOLYTIC REPLICATION OF E1B-DELETED ADENOVIRUSES
}

\author{
By \\ Pei-Hsin Cheng
}

B.S., National Sun Yat-Sen University, 2005

M.S., National Sun Yat-Sen University, 2007

M.S., University of Louisville, 2011

\begin{abstract}
A Dissertation
Submitted to the Graduate Faculty of the University of Louisville School of Medicine in Partial Fulfillment of the Requirements

for the Degree of
\end{abstract}

Doctor of Philosophy

Department of Pharmacology and Toxicology

University of Louisville

Louisville, Kentucky

December 2013 

CYCLIN E INDUCTION AND ONCOLYTIC REPLICATION OF E1B-DELETED ADENOVIRUSES

$$
\text { Pei-Hsin Cheng }
$$

B.S., National Sun Yat-Sen University, 2005

M.S., National Sun Yat-Sen University, 2007

M.S., University of Louisville, 2011

A Dissertation Approved on

November 22, 2013

by the following Dissertation Committee:

Kelly McMasters, M.D., Ph.D.

Heshan Zhou, Ph.D.

Donald Nerland, Ph.D.

David Powell, Ph.D.

Wolfgang Zacharias, Ph.D. 


\section{DEDICATION}

This dissertation is dedicated to my dear grandfather Mr. Jin-Yuan Lin, who always encouraged me to fearlessly pursue my dream in an unfamiliar land until the end of his life. His unconditional love, support and encouragement are still as vivid today as when we were together, though he was not able to see his beloved granddaughter become the first Ph.D. in the family with his own eyes. I pray for him to rest in peace and I want him to know that I have gone this far to honor him. 


\section{ACKNOWLEDGMENTS}

First, I would like to thank my mentors, Drs. Kelly McMasters and Heshan Zhou.

The leadership and being a role model for me from Dr. McMasters and the daily mentoring, as well as faithfully believing in my ability from Dr. Zhou, all in all have fostered my strengths and professional development. Without the wholeheartedly support, trust and guidance from my mentors, it would never be possible for me to accomplish this work. I would also like to thank my dissertation committee members, Drs. Donald Nerland, David Powell, and Wolfgang Zacharias, for their critical comments and valuable suggestions on my dissertation. Besides the time they served in the annual meetings, their supports, encouragements and words of wisdom also aided my professional development and served beyond their scientific contributions as the regular dissertation committee members.

My thanks are extended to the past and current members in the laboratory, including Drs. Aracely Garcia-Garcia, Deyi Xiao, Humberto Rodriguez-Rocha, Hongying Hao, Jorge Gomez-Gutierrez, Lan Chen, Michael Egger and Xiao-Mei 
Rao, along with Mr. Stephen Wechman for their technical support, valuable suggestions and invaluable friendship. I would also like to thank the members in Dr. Xiao-Feng Li's laboratory, including Drs. Xiao-Feng Li and Xiaoxian Duan, for their patience and assistance in animal studies and immunohistochemistry. I especially thank Stephen Wechman for being my language partner, unconditionally helping me with crucial suggestions in editing my scientific writings and documents. My sincerest gratitude goes to my Louisville family, Dr. Rao and her family, for their continuous love, moral support and precious fellowship.

I would like to express my deepest appreciation to Dr. Theresa Chen for affording me the opportunity to apply for Integrated Programs in Biomedical Sciences in University of Louisville. Without her effort, I would never be able to sit in front of the computer, typing these words in my dissertation acknowledgement at this special moment of reflection. She also served as my personal mentor, providing profound guidance and precious advice to both of my professional and personal life. I am also grateful to have my friends Feifei Song, Florence Su, Pastor Moss Wu and all the friends in Louisville Taiwanese Presbyterian Church for their 
fellowship and constant praying for God's glorious grace bestowed on me. I am very fortunate to have my ladies, Chi Zhang and Yanfang Zhu, who served as the most incredible support system throughout my time in Louisville. The voyage to the American dreamland is extremely tough for a foreign individual. How luckily I am, to have you always on my side, sharing the happiness and pain, as well as encouraging me to never give up. Special appreciation goes to my beloved family, my grandmother Mrs. Xiu-Tao Chen Lin, my parents Mr. Jen-Huei Cheng and Mrs. Feng-Chuang Lin, my brother Yu-Che Cheng, and my cousin Chiying Cheng, together with her family for supporting me with endless love, careful guidance and assistance. Because of you, providing me matchless strength and courage, I know I can always explore the unknown fearlessly and pursue my career in science.

I am only afraid that words are too short to convey my deepest gratitude. With my sincerest appreciation, I wish all of you can understand that without you, I would not be able to pursue my dreams and achieve what I have here. 


\section{ABSTRACT \\ CYCLIN E INDUCTION AND ONCOLYTIC REPLICATION OF E1B-DELETED ADENOVIRUSES \\ Pei-Hsin Cheng}

November 22, 2013

Virus-mediated oncolysis has been considered as a new and promising cancer therapeutic approach. Although adenoviruses (Ads) with deletion of E1b55K preferentially replicate in cancer cells and have been used in numerous cancer treatments, the selective replication mechanism of this kind of virus still remains controversial. The lack of a well-established studies focusing on possible mechanisms enabling tumor selectivity of oncolytic Ads has hindered the further development of virotherapies and limits their clinical applications. Therefore, uncovering the molecular basis behind the tumor-killing phenomena will fill critical gaps in our understanding of the oncolytic adenovirology. Previously our laboratory has demonstrated that Ad E1B55K protein is involved in cyclin E induction that is required for efficient virus replication. However, functional E1B55K expression is not required for oncolytic Ad replication in cancer cells, many of which have deregulated cyclin E overexpression. In this dissertation we advanced the previous findings to further develop the mechanism model of selective oncolytic replication of $E 1 b$-deleted Ads, and then applied these insights 
to design a novel tumor-specific oncolytic Ad vector. We demonstrated that Ad-induced cyclin E subsequently activates CDK2 that targets the transcriptional suppressor $\mathrm{pRb}$ to generate a cellular environment for productive viral replication. Based on this understanding, a novel tumor-specific oncolytic vector, Ad-cycE, was developed in which the cyclin E promoter was used to control a critical regulatory viral E1a gene. As cyclin E is greatly induced in cancer cells after Ad transduction, Ad-cycE shows significant oncolytic efficacy in vitro and strongly repressed tumor growth in vivo. Additional work has been completed exploring the impact of using a combination treatment of Ad-cycE and rapamycin which induces autophagy; the results indicated a synergistic antitumor effect that was statistically significant. This work has advanced our knowledge of the cancer selectivity of E1b-deleted Ads, and will lead to further progress in the field of oncolytic virotherapy. 


\section{TABLE OF CONTENTS}

PAGE

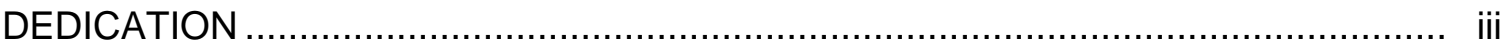

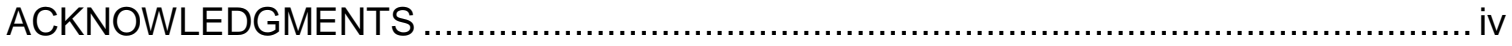

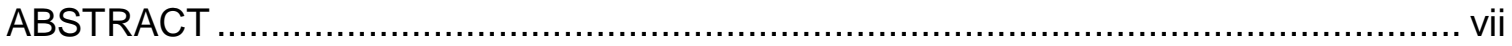

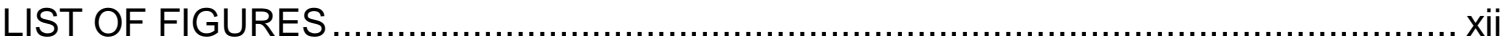

CHAPTERS

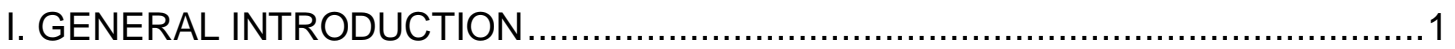

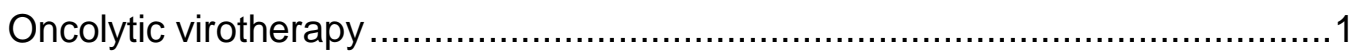

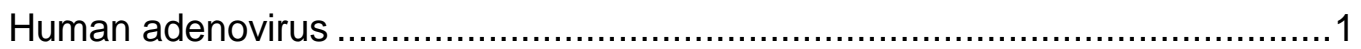

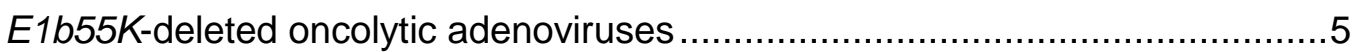

Molecular mechanisms of E1b55K-deleted Ad selectivity in cancer cells...........7

Cancer selectivity of E1b55K-deleted Ads based on p53 deficiency ..................7

Cancer selectivity of E1b55K-deleted Ads based on late viral mRNA export ...12

Cancer selectivity of E1b55K-deleted Ads based on cyclin E or cell cycle

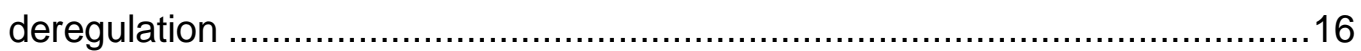

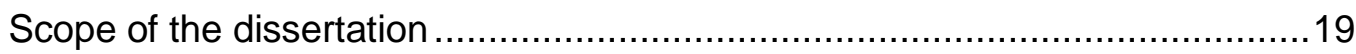

II. MOLECULAR BASIS FOR VIRAL SELECTIVE REPLICATION IN CANCER CELLS: ACTIVATION OF CDK2 BY ADENOVIRUS-INDUCED CYCLIN E 
Introduction

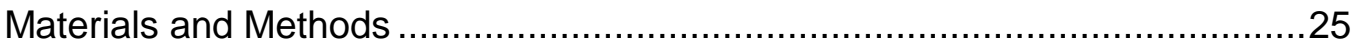

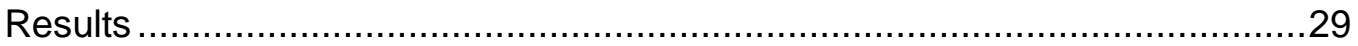

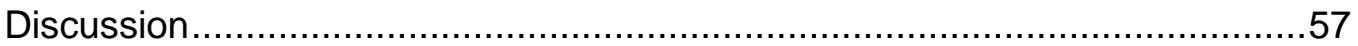

III. ONCOLYTIC ADENOVIRUS TARGETS CYCLIN E OVEREXPRESSION AND INHIBITS TUMOR DEVELOPMENT IN VIVO

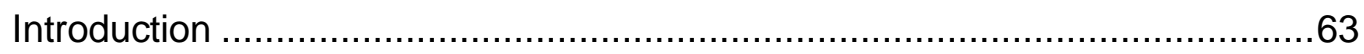

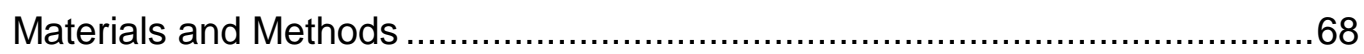

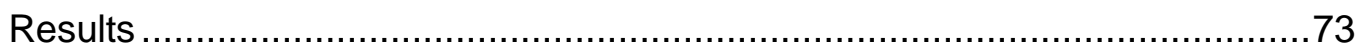

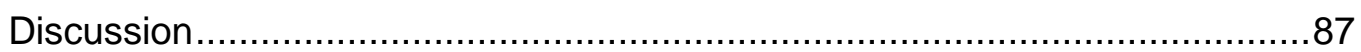

IV. PROPERTIES OF ONCOLYTIC REPLICATION OF ADENOVIRUSES IN MURINE AND HUMAN LUNG CANCER CELLS

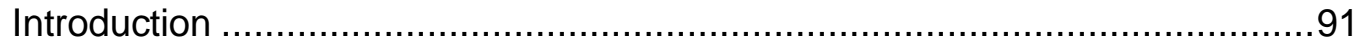

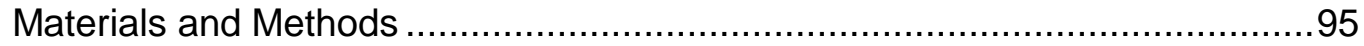

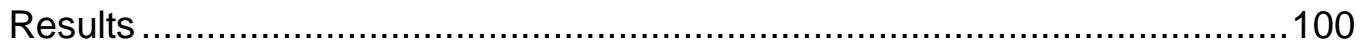

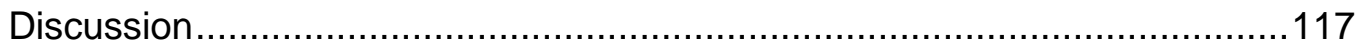

V. COMBINATION OF AUTOPHAGY INDUCER RAPAMYCIN AND ONCOLYTIC ADENOVIRUS IMPROVES ANTITUMOR EFFECT IN CANCER CELLS

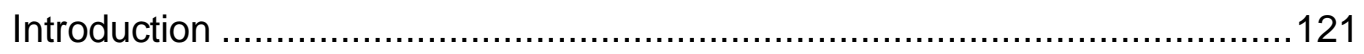

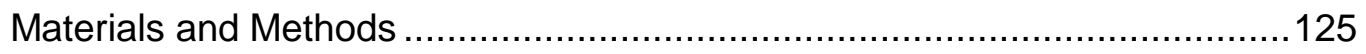

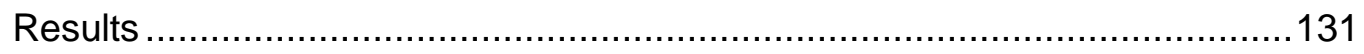




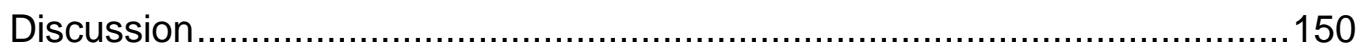

CHAPTER VI. SUMMARY AND FUTURE PERSPECTIVES ................................155

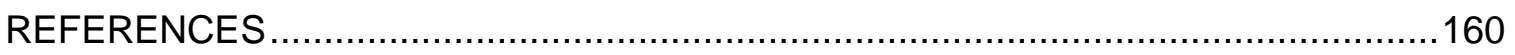

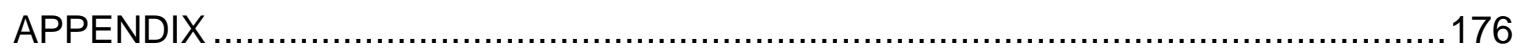

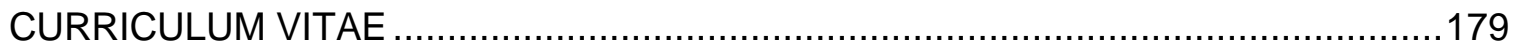




\section{LIST OF FIGURES}

PAGE

1.1. Generalized structure and genome map of adenovirus serotype 5.

1.2. Proposed mechanism that oncolytic selectivity of E1b55K-deleted Ads depends on p53 deficiency in cancer cells.

1.3. Proposed mechanism that late viral mRNA export determines oncolytic selectivity 15

1.4. Proposed mechanism that cyclin $E$ deregulation in cancer cells is the molecular basis of oncolytic selectivity 18

2.1. Virus replication is correlated with cyclin $E$ overexpression

2.2. Cyclin $\mathrm{E} / \mathrm{CDK} 2$ complex induced by viral infection in $\mathrm{A} 549$ cells 35

2.3. Effects of viral replication on cellular proteins related to $\mathrm{G} 1 / \mathrm{S}$ phase 38

2.4. Effects of viral replication on $\mathrm{pRb}$ and $\mathrm{CDK}$ inhibitors

2.5. Effects of roscovitine on CPE and viral production.

2.6. Effects of Ros on viral DNA synthesis, viral capsid proteins, virus-induced cyclin $E$ and phospho-pRb S612

2.7. Effects of CDK2-specific siRNA on Ad replication in A549 human lung cancer cells...

2.8. Effects of CDK2-specific siRNA on Ad replication in WI-38 human lung fibroblast cells

2.9. Proposed mechanism of cyclin E function in Ad replication. 
3.1. Proposed rationale of activating E1A transcription in Ad-cycE vector.

3.2. Comparison of prostate-cancer-promoter-driven Adhz55 and cyclin E-promoterdriven Ad-cycE in prostate cancer cells

3.3. Cytopathic effects of human Ads on WI-38 and A549 cells

3.4. The expression of viral E1A and virus production in A549 cells .79

3.5. Cytotoxicity of Ad-cycE in multiple cancer cell types .81

3.6. In vivo antitumor effects of Ad-cycE on subcutaneous A549 human lung cancer in nude mice. .85

4.1. Growth characteristics of A549 and ED-1 cells 101

4.2. Infection efficiency of human adenoviruses on A549 and ED-1 cells 103

4.3. The DNA amounts of AdGFP entering cells. 105

4.4. Cyclin E expression and cytopathic effects of human Ads on human A549, murine ED-1, and NIH/3T3 cells 108

4.5. Burst ratios of human Ad replication in ED- 1 and NIH/3T3 cells 111

4.6. Human Ad DNA synthesis in A549 and ED-1 cells 113

4.7. The expression of viral E1 A, capsid protein and virus production in A549 and ED-1 cells.

5.1. Structure of the Ads.

5.2. Selective oncolytic replication of $\mathrm{Ad}-\mathrm{cyc} \mathrm{E}$ 134

5.3. Effects of rapamycin on cytotoxicity and autophagy. 137 
5.4. Effects of combination of rapamycin and Ad-cycE on A549 cells

5.5. Effects of rapamycin on the oncolytic replication of Ad-cycE

5.6. Analysis by Calcusyn (Biosoft, Ferguson, MO) of the interaction between rapamycin

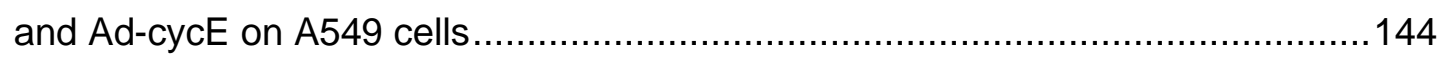

5.7. Effects of combination of rapamycin and Ad-cycE on MDA-MB-231 cells 147

5.8. Effects of combination of rapamycin and wild-type Ad on A549 cells .149 


\section{CHAPTER I}

\section{GENERAL INTRODUCTION}

\subsection{Oncolytic virotherapy}

Cancer is the most common cause of death in the world (1). The limitations of currently available therapies demand the continued development of more potent cancer treatment options. Oncolytic virotherapy represents a fast growing therapeutic platform for cancer treatment $(2,3)$. The therapeutic effects emanate from a relatively small amount of virus, which preferentially replicates in and lyses cancer cells. This is followed by a localized spread to the surrounding tumor cells, ultimately leading to reduction of the tumor mass (4). Current oncolytic viruses, including adenovirus (Ad), herpes simplex virus, measles virus, newcastle disease virus, reovirus, parovirus, poliovirus, seneca valley virus, retrovirus, vaccinia, and vesicular stomatitis virus, have been tested in numerous preclinical or clinical settings (5). Although many viruses have already been developed, Ads are still the most commonly used oncolytic vectors for gene therapy due to their infection efficacy, high titer production, safety, easy genetic modification and well-studied replication characteristics (6).

\subsection{Human adenovirus}

Human adenoviruses, as a common cause of respiratory tract diseases, are 
nonevenloped DNA viruses which can infect cells at many different cell cycle stages without integrating into host cellular chromosomes (7). Adenovirus serotype 5 (group C) have been wildely used in oncolytic virotherapy due to the favorable safety profile and only cause clinical disease in patients by producing negligible flu-like symptoms such as fever, myalgias, asthenia and chills $(8,18)$. Generalized Ad structure and viral genome map were depicted in Figure 1.1. The viral genome is composed of a linear, double-stranded DNA of approximately 36 $\mathrm{Kb}$ and can be divided into early $(E)$ and late $(L)$ genes $(7,9)$. The $E 1$ genes encode critical proteins including $E 1 a$ and $E 1 b$ for the initiation and regulation of viral and cellular gene expression. The E2 genes encode three different proteins which function directly in viral DNA replication, while the E3 genes encode proteins which can modulate infected host immune responses. Products of the $E 4$ genes encode proteins of diverse functions such as regulating viral gene transcription, translation, mRNA nuclear export and apoptosis pathways. The $L$ genes encode structural proteins for packaging the viral genome into virion particles during the final stages of virus replication. 


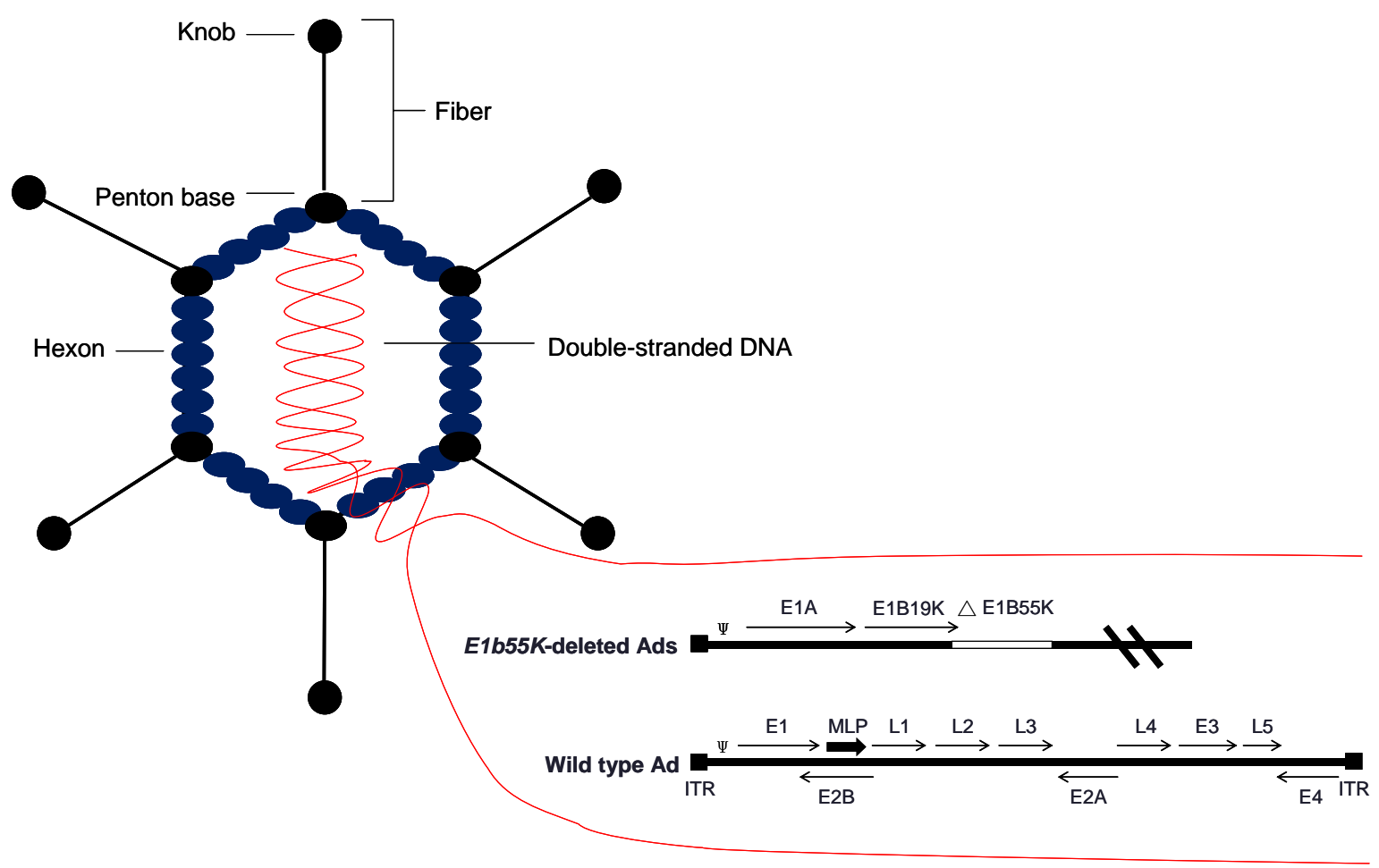

Figure 1.1. Generalized structure and genome map of adenovirus serotype 5. Major structural components of a wild-type Ad capsid include hexon, penton base, and fiber structures. Adenovirus capsids enclose a $36 \mathrm{~Kb}$ double-stranded DNA genome, shown as the red line inside the capsids. The inverted terminal repeat (ITR), the packaging signal ( $\Psi$ ) and major late promoter (MLP) are indicated. The solid lines represent viral genome and the white line represents the deleted region. Early transcripts are represented by E1-E4 regions and late transcripts are represented by L1-L5 regions. E1 region contains $E 1 A$ and $E 1 B$ regions. The E1B19K and E1B55K in E1B region have a small overlap. 
The Ad infection begins by binding of knob domain on the adenovirus fiber to a specific coxsackievirus and adenovirus receptor (CAR) located on the cell surface; this interaction facilitates the entry of virus into the host cells by endocytosis. The low-pH environment of the endosome allows the viral capsid to disassociate, resulting in the release of virions from the endosome (10-12). By cooperating with microtubules, virions are then transported to the nuclear pore complex where the virions disassemble and the double-stranded viral DNA is released to directly enter the nucleus.

The replication of Ads relies on utilizing the cellular components as building blocks. The replication cycle of Ads is divided into two phases based on the onset of viral gene expression (7). The early phase includes the expression of a set of viral genes to promote cellular entry into an S-like phase, block apoptosis, and prevent cellular immune responses. After the early gene products adjust the replication machinery and other critical cellular components inside host cells, the gene expression transitions to the late phase, in which adenoviral DNA replicates and the structural proteins are synthesized. Once adenoviruses have finished the amplification of their DNA and synthesis of their structural proteins, then viral assembly can take place producing virions. Viral replication subsequently cause cell lysis and release of progeny virions from the infected cells, leading to significant amplification of the Ads which produces up to 10,000 viral particles per cell $(7,13)$. 


\subsection{E1b55K-deleted oncolytic adenoviruses}

The idea of treating cancers with the adenoviruses began in the 1950 s $(5,14)$. Wild-type Ad5 was injected in thirty patients with epidermoid cervical carcinomas and $65 \%$ of the virus injections induced necrosis in the tumors without causing severe toxicity. While producing some promising results, the long-term safety concerns, limited final therapeutic efficacy and tumor recurrence after the treatment was completed prevented further development of this approach until recently. A novel gene-attenuated oncolytic adenovirus dl1520 (ONYX-015) was designed with an 827-bp deletion in the $E 1 b$ region and a point mutation which generates a premature stop codon to prevent the expression of its E1B55K protein (Fig 1.1) (15-17). This new virus dl1520 showed potent and promising oncolytic efficacy in several preclinical studies and became the first oncolytic adenovirus used in the clinical trials in United States since 1996, treating approximately 200-300 cancer patients with various routes of administration in more than ten clinical trials (from phase I to II) $(17,18)$. However, during a phase III clinical trial in 2003, when treatment with dl1520 was combined with chemotherapy in patients with head and neck squamous cell carcinoma the trial was suspended due to limited therapeutic potential in metastatic cancer patients (18).

H101 (Oncorine) is another oncolytic adenovirus similar to dl1520 generated by the Chinese company, Sunway Biotech $(18,19)$. The phase I clinical trial with $\mathrm{H} 101$ as a single therapeutic agent began in 2000 in China and the phase II and III trials of H101 in combination with chemotherapy started from 2001 to 2004 . 
Overall, the clinical tolerability and responses of $\mathrm{H} 101$ treatment have been very promising $(18,19)$. The rights to dl1520 were bought by Sunway Biotech which has completed Phase III clinical studies. More than 600 cancer patients have safely received the treatment with $\mathrm{H} 101$ in clinical trials in China $(18,20)$. The company reported a $79 \%$ positive response rate for $\mathrm{H} 101$ plus chemotherapy as compared with $40 \%$ for chemotherapy alone (21). Considering the impact of these results, the Chinese State Food and Drug Administration approved $\mathrm{H} 101$ for use in combination with chemotherapy for the treatment of late-stage cancers. Therefore, Sunway Biotech has marketed $\mathrm{H} 101$ for treating head and neck cancers $(21,22)$. Besides $\mathrm{H} 101$, this company has also developed other genetically modified oncolytic adenoviruses $\mathrm{H} 102$ and $\mathrm{H} 103$ for cancer treatment (23). $\mathrm{H} 102$ virus replication is driven by the alpha-fetoprotein promoter which specifically targets primary hepatocellular carcinoma, and is currently in the preclinical stage of development. $\mathrm{H} 103$ is an oncolytic adenovirus carrying a tumor antigen known as heat shock protein (HSP) 70 gene which can stimulate an antitumor immune response while the virus selectively replicates within tumor cells. A phase I clinical trial of intratumoral injection of $\mathrm{H} 103$ has been conducted in a total of 27 patients with advanced stage solid tumors; an $11.1 \%$ objective response (cases with complete response + partial response) to $\mathrm{H} 103$-injected tumors and a $48.1 \%$ clinical benefit rate (cases with complete response + partial response + minor response + maintain a stable disease) was reported in 2008 (20). 
Currently various oncolytic adenoviruses have been reported in preclinical studies and at least ten of these have been active or approved for human clinical trials ( 3 , 5). Examples include Ad-OC-E1a (24), Ad5-CD/TKrep (25, 26), Ad-delta24-RGD (27, 28), CG7060 (CV706) (29), CG7870 (CV787) (30), CG0070 (31), Telomelysin (OBP-301) (32), CGTG-102 (33), H103 (20), KH901 (34) and ICOVIR-5 (currently recruiting patients, Clinicaltrials.gov identifier: NCT01864759). These vectors were constructed based on the deletion of critical viral genes combined with multiple strategies such as transcriptionally targeting cancer cells with tumor-specific promoters, or transductionally targeting cancers with modification on various viral capsid proteins, or arming the vectors with immune stimulating genes (35). Nevertheless $\mathrm{H} 101$ is the only oncolytic adenovirus that has been commercially approved for clinical application in China, but not in the other countries.

\subsection{Molecular mechanisms of E1b55K-deleted Ad selectivity in cancer cells} Even though E1b55K-deleted oncolytic Ads have been used in several clinical trials and is marketed in China, the mechanism(s) enabling this oncolytic selectivity, remains controversial. The following sections summarize three proposed mechanisms.

\subsubsection{Cancer selectivity of E1b55K-deleted Ads based on p53 deficiency}

The original mechanism proposed that E1b55K-deleted oncolytic Ad could only replicate in p53-deficient tumor cells, but not in normal cells with functional p53 
(Fig. 1.2) $(16,17,36)$. After virus infection, Ad E1A is expressed immediately to regulate expression of viral genes and promote transduced cell entry into an S-like cell cycle phase (37). In response to Ad infection, host cells have developed a crucial strategy to block the virus spread by activation of p53-mediated apoptosis and cell-cycle arrest after Ad infection and E1A expression (7, 38, 39). Ad E1A triggers the accumulation of p53 protein either by activating p53 transcription, (40) or stabilizing p53 via inducing expression of p14 ${ }^{\mathrm{ARF}}$ tumor suppressor (referred to as $\mathrm{p} 19^{\mathrm{ARF}}$ in murine cells) which binds to MDM2 protein and subsequently blocks MDM2-induced p53 degradation and transactivational silencing (41-43). Consequently, high levels of p53 in infected cells lead to either cell-cycle arrest or apoptosis to block viral replication and spread.

In response to this, Ad has developed its ways to overcome p53-mediated apoptosis and cell-cycle arrest triggered by Ad infection. It was reported that Ad E1B55K protects the infected cells from the E1A-induced p53 effects through at least three distinct mechanisms. First, E1B55K binds to the amino terminus of p53, and thus represses p53 transactivation $(44,45)$. Second, E1B55K cooperates with Ad E4orf6 to proteolytically degrade p53 (46-48). Third, E1B55K alone can function as an E3 SUMO1-p53 ligase eventually also leading to p53 polyubiquitinylation and proteasomal degradation (49). Therefore in normal cells, E1B55K counteracts the p53-dependent apoptosis induction by E1A and prevents premature cell death, resulting in efficient viral replication $(44,45)$. Without E1B55K protein, E1b55K-deleted Ads were considered to not be able to 
counteract E1 A-induced p53 accumulation and thus fail to replicate in normal cells with functional p53 protein. Deficiency of p53 occurs frequently in many types of human cancers due to $p 53$ gene deletion or mutation $(50,51)$. Since cancer cells typically lack functional p53 protein or its pathways, the E1B55K function of inhibiting p53 is not as important as it is in normal cells and thus it was proposed that dl1520 could selectively replicate in cancer cells with dysfunctional p53 pathways $(16,17)$.

While some reports have supported the hypothesis that E1b55K-deleted Ads selectively kill cancer cells with p53 deficiency, the original mechanism has been challenged by several studies. Studies in our laboratory and others' have shown E1b55K-deleted Ads are able to replicate in and kill cancer cells with wild-type p53 as efficiently as in cancer cells with p53 deficiency (52-56). To resolve this paradox, it was proposed that either p53 deficiency or p14 ARF deficiency might determine the cancer selectivity of E1b55K-deleted Ads $(57,58)$. In cancer cells with wild-type $\mathrm{p} 53$, loss of the functional $\mathrm{p} 14^{\mathrm{ARF}}$ would neutralize MDM2-mediated p53 degradation and was considered to prevent p53 from its normal functions. Thus $\mathrm{p} 14^{\mathrm{ARF}}$ deficiency in cancer cells was indicated as the alternative molecular mechanism to allow dl1520 replication in these cancer cells with wild-type p53. However, experimental data contradicted this mechanism by showing that in several cancer cell lines the replication of dl1520 is not controlled by the p53 or p14 ${ }^{\mathrm{ARF}}$ status (59). 
Also, it has been reported that in some cell lines, p53 can promote the viral lytic cycle and may be required for productive virus replication and late viral gene expression by cooperating with $\mathrm{E} 1 \mathrm{~A}$ to enhance transcription at the major late gene promoter of the virus genome $(60,61)$. Further studies also revealed that accumulation of $\mathrm{p} 53$ induced by $E 1 \mathrm{~b} 55 \mathrm{~K}$-deleted or E1b55K-mutated Ads can neither efficiently induce apoptosis nor activate the transcription of downstream p53-responsive genes in Ad-infected primary cells $(62,63)$. Moreover, in cells which are infected with E1b55K-deleted Ads, E1b19K, as a homologue of BCL-2 family members, can also function as an inhibitor to adenovirus E1A protein-induced apoptosis (38). Even if E1A-induced apoptosis occurs in Ad-infected cancer cells, it does not prevent the replication of E1b-deleted oncolytic Ads in the majority of cells (38). Thereby, blocking p53 activity may not be the major requirement for viral replication, and p53 deficiency in cancer cells seems unlikely to be the determinant of oncolytic selectivity of E1b55K-deleted Ads.

So far none of the comprehensive research has been reported to sort out the controversy existing between this mechanism model and currently available literature. The lack of new experimental data to support the original hypothesis hence raises the concern that the mechanism of cancer selectivity of E1b55K-deleted oncolytic Ads has to be further addressed. 


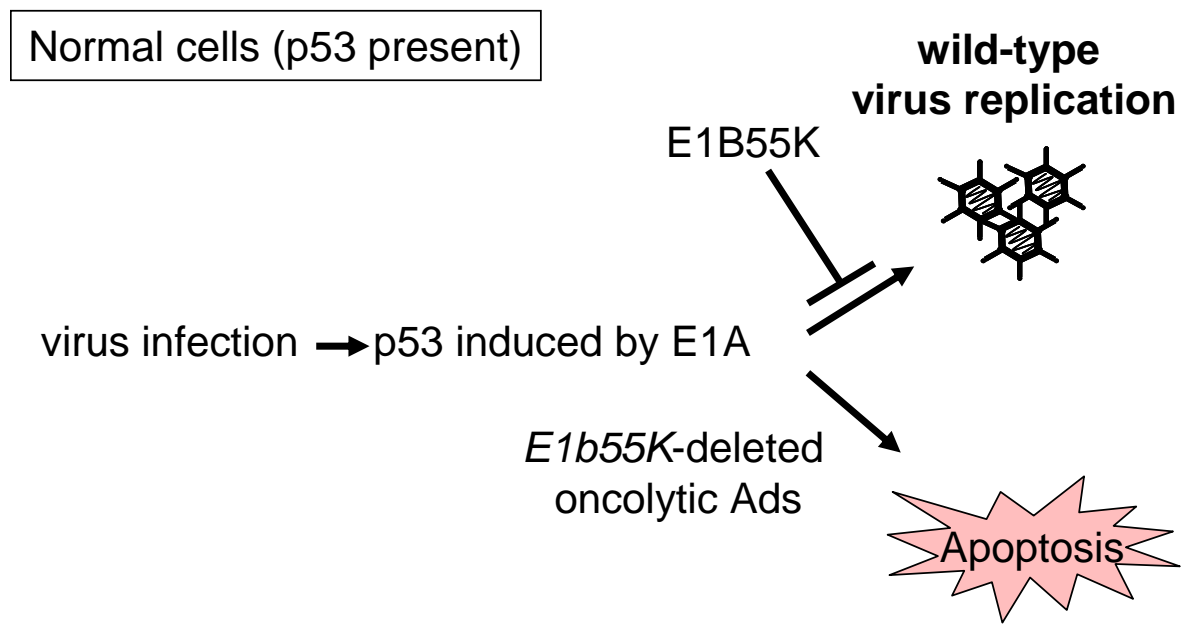

Cancer cells ( $p 53$ deficient)

E1b55K-deleted oncolytic Ad replication virus infection $\rightarrow$ p53 induced by E1A $\rightarrow$

Figure 1.2. Proposed mechanism that oncolytic selectivity of E1b55K-deleted Ads depends on p53 deficiency in cancer cells. E1B55K is reported to inhibit p53 function, which prevents cellular p53-dependent apoptosis induced by $\mathrm{E} 1 \mathrm{~A}$, and thus allows virus to replicate. In normal cells with p53 expression, E1b55K-deleted oncolytic Ads do not contain E1B55K to counteract E1A-induced p53 accumulation. The p53-mediated cell apoptosis therefore prohibits E1b55K-deleted oncolytic Ads to replicate in those cells. However, in p53-deficient cancer cells, lack of p53 expression allows oncolytic Ads to replicate in the cells without undergoing E1A-induced apoptosis. 


\subsubsection{Cancer selectivity of E1b55K-deleted Ads based on late viral mRNA export}

The mechanism that permissive cancer cells provide the late viral RNA export functions for E1b55K-deleted Ads to carry out sufficient oncolytic replication was proposed by O'Shea et al. (2004) (63) (Fig, 1.3). During the virus replication process, the levels of late viral mRNAs raise in cytoplasm for translation whereas the accumulation of cellular mRNAs in cytoplasm is blocked (7). E1B55K functions in a complex with the viral E4orf6 protein to direct the switch from host to viral protein synthesis by promoting the preferential nuclear export and translation of the late viral mRNAs to cytoplasm (63-68). Several lines of evidence support the hypothesis that the E1B55K/E4orf6 complex is directly involved in the selective nuclear export of late viral mRNAs through active nucleocytoplasmic shuttling via the exportin $\mathrm{CRM} 1(7,64,69)$ or through its E3 ubiquitin-protein ligase activity $(68,70,71)$. However, the molecular mechanism(s) of either selective nucleocytoplasmic transport mediated by E1B55K/E4orf6 complex or the assembly and activity of the E3 ubiquitin-protein ligase associated with E1B55K/E4orf6 complex has not been fully understood (70). Since E1b55K-deleted oncolytic Ads lack E1B55K to mediate late viral mRNA export from nucleus in primary cells, the viruses fail to efficiently replicate in those normal cells (63). Permissive cancer cells, however, have a propensity to support the nuclear export of viral late mRNAs in the absence of E1B55K while normal cells do not, allowing oncolytic replication of E1b55K-deleted oncolytic Ads to occur. 
O'Shea et al. (2005) further reported that either restoring viral L4 100K protein expression or inducing heat shock responses can partially rescue E1b55K-deleted Ad replication in refractory cancer cells (72). In contrast to permissive cancer cell lines, for E1b55K-deleted Ad replication, refractory cancer cell lines fail to offer preferential nuclear export and translation of viral late mRNAs including Ad L4 100K mRNA in the absence of E1B55K (72). Ad L4 100K is known to be involved in host protein shutoff and promote preferential nuclear export and translation of viral late mRNAs (73-75). As a consequence of the absence of E1B55K, lack of L4 100K expression in refractory cancer cells leads to the poor oncolytic Ad replication and ectopic expression of L4 100K in refractory cancer cells enhanced the expression of late viral proteins. In the same report the authors also implied that heat shock proteins overexpressed in various human cancers may be the important molecular mechanism for cancer cells to support the differential transport of late viral mRNA export between normal and cancer cells. The cellular responses to heat shock resemble the late stages of adenovirus infection with the inhibited translation of cellular mRNAs and preferential translation of heat shock mRNAs. Also it has been shown that the 5'UTR of mammalian heat shock protein (Hsp) 70 and late adenoviral mRNAs share structural homology, which may contribute to both selective translation and nuclear export through a common mechanism (76). Thus induction of heat shock responses by physical or pharmacological means could selectively rescue the export of late viral mRNAs in refractory cancer cells, rendering them once again permissive to E1b55K-deleted Ad replication. 
However, although expression of L4 $100 \mathrm{~K}$ and inducing heat shock responses may participate in determining the cancer selectivity of E1b55K-deleted Ads on late viral mRNA export, limited evidence has also shown neither expressing $L 4$ $100 \mathrm{~K}$ nor inducing heat shock responses could completely restore E1b55K-deleted Ad replication to the level of wild-type Ad in a majority of cancer cell lines. Since ectopic expression of L4 $100 \mathrm{~K}$ and inducing heat shock responses cannot recover wild-type replication capacity in the absence of E1B55K, the importance of E1B55K for selective oncolytic Ad replication cannot be ignored. The exact mechanics of the interaction of E1B55K, L4 100K, and heat shock responses to regulate mammalian and late viral mRNA export which leads to cancer selectivity of E1b55K-deleted Ads still required for further studies. Additionally, Gonzalez et al. (2006) indicated that it appears overly simplistic to generalize that the cancer selectivity of E1b55K-deleted Ads relies on the efficiency of viral late mRNA export (77). E1B55K has many more functions than regulating the differential export of mRNAs, that are important for Ad replication and therefore the outcome of a complex interaction between E1B55K and multiple cellular components should be considered holistically in determining the cancer selectivity mechanism. 


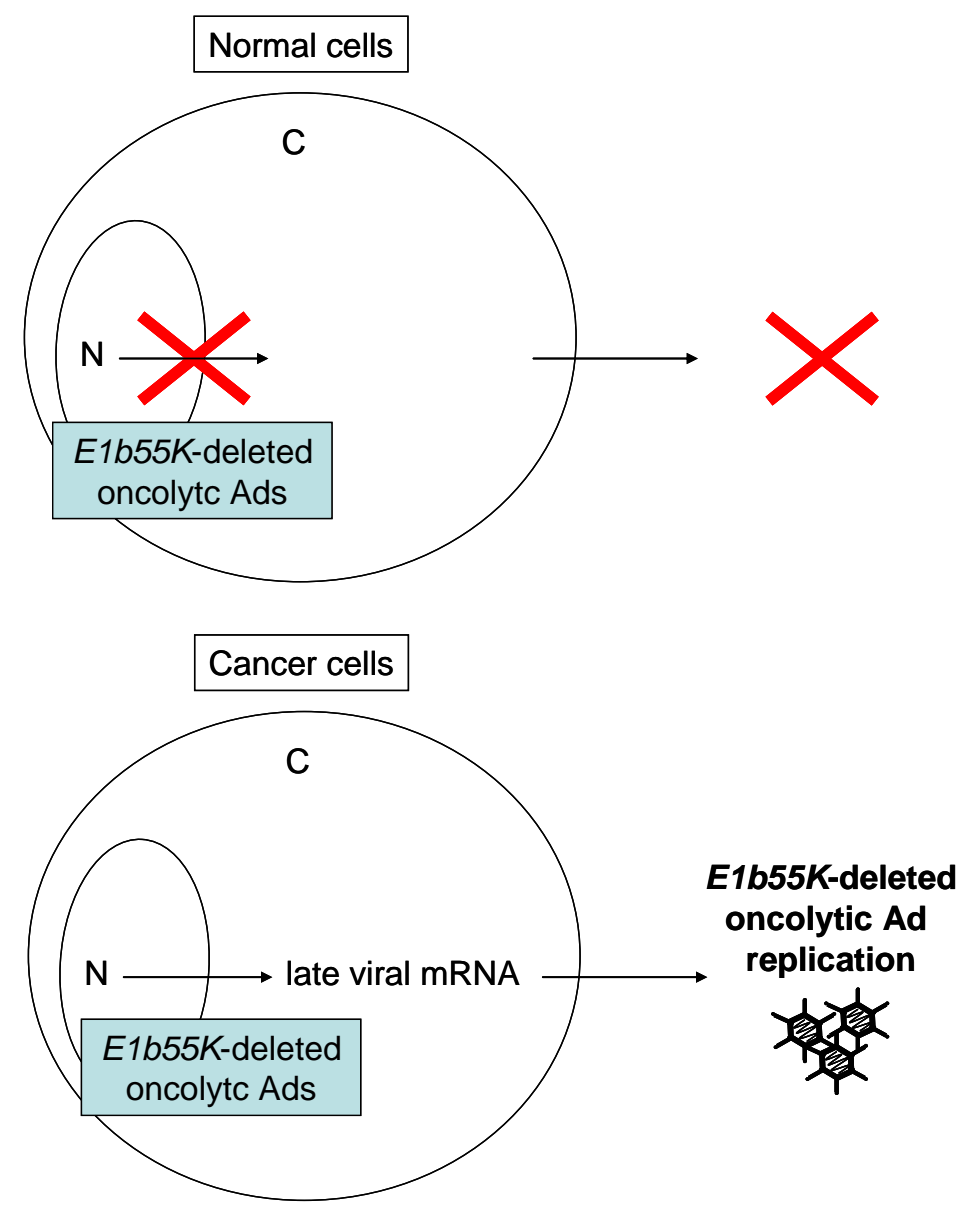

Figure 1.3. Proposed mechanism that late viral mRNA export determines oncolytic selectivity. "N" stands for nucleus and "C" stands for cytoplasm. E1B55K/E4orf6 complex mediates the preferential export of late viral mRNAs from cell nucleus $(\mathrm{N})$ to cytoplasm $(\mathrm{C})$ to carry out the translation. In normal cells, a defect in late viral mRNA export of E1b55K-deleted Ads leads to the failure of sufficient virus production. Cancer cells with properties to rescue this export and expression of late viral mRNA to allow the oncolytic replication of $E 1 b 55 K$-deleted Ads. 


\subsubsection{Cancer selectivity of E1b55K-deleted Ads based on cyclin E or cell cycle deregulation}

Studies conducted by Goodrum and Ornelles (1999) showed that the E1B55K function in late viral mRNA export may not correlate with the restricted replication of $E 1 \mathrm{~b} 55 \mathrm{~K}$-deleted Ads in cells under $\mathrm{G}_{0} / \mathrm{G}_{1}$ phase (67). Unlike wild-type Ad which is able to efficiently produce viral progeny regardless the cell-cycle stages, the replication of $E 1 b 55 K$-deleted Ads is restricted in HeLa cells under $G_{0} / G_{1}$ phase but less restricted in cells under $\mathrm{S}$ phase. Yet the total amount of cytoplasmic late viral mRNA was found to be greater in cells infected during $\mathrm{G}_{1}$ phase than that in cells infected during S phase with either the wild-type or E1b55K-deleted Ads. A study further suggested that the E1B55K protein has a property to enable the virus to overcome the growth restrictions to its replication imposed by the cell-cycle stage (78).

Based on our study with large-scale gene arrays, we found that viral $E 1 b$ products affected the expression of numerous genes involved in cell cycle regulation, transcription, apoptosis, protein metabolism, stress responses and angiogenesis (79). Most importantly, the levels of some key regulators of cell cycle progression, including cyclin E and CDC25A, were significantly increased by E1B.

Cyclin E is well-characterized as a critical cell cycle protein to promote $G_{1} / S$ phase transition $(80,81)$ in either cyclin-dependent kinase 2 (CDK2)-dependent (82) or CDK2-independent manner $(83,84)$. Expression of cyclin E is strictly 
controlled in normal cells while the deregulation of cyclin $E$ is highly related to tumorigenesis (85-88). Constitutive overexpression of cyclin $\mathrm{E}$ can induce chromosome instability and impair normal cell cycle progression $(89,90)$. Cyclin E gene amplification (91), overexpression of cyclin E mRNA or protein levels (87, 92), decrease of cyclin $E$ turnover (93), together with the presence of more active forms of cyclin E (94-96) have been reported in many types of cancers, such as breast, gastrointestinal, lung and skin cancers.

We have reported that $\mathrm{E} 1 \mathrm{~B} 55 \mathrm{~K}$ has a novel function to induce cyclin $\mathrm{E}$ expression which is critical for virus replication $(56,79)$. The replication of $E 1 b 55 K$-deleted oncolytic Ads is restricted in normal cells due to the failure to induce cyclin E. Cancer cells, with the cyclin E deregulation, may provide the E1B55K-like factors to support cyclin E induction by E1b55K-deleted Ads, leading to sufficient oncolytic replication. We thus proposed that cyclin E deregulation in cancer cells is a molecular basis of oncolytic selectivity of E1b55K-deleted Ads (Fig. 1.4.). Permissive cancer cells may provide E1B55K-like factors to relax cyclin E regulation and allow E1b55K-deleted Ads to induce cyclin E expression similarly to wild-type Ad in cancer cells, but not normal cells, leading to preferential oncolytic replication. Ad-induced cyclin E subsequently activates CDK2 which inhibits the transcriptional suppressor $\mathrm{pRb}$ to generate a cellular environment for viral productive replication. 


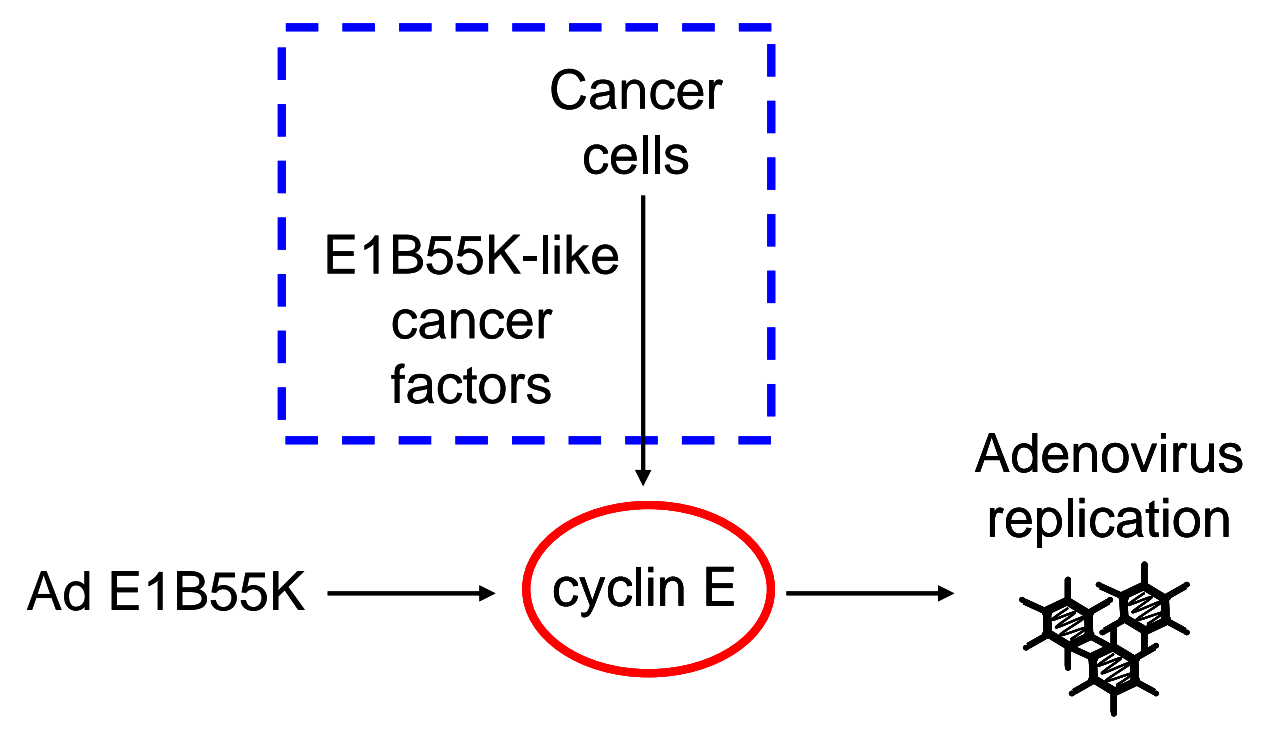

Figure 1.4. Proposed mechanism that cyclin E deregulation in cancer cells is the molecular basis of oncolytic selectivity. In Ad-infected normal cells, Ad E1B55K functions to enhance cyclin E expression for virus replication. Without E1b55K to induce cyclin E, E1b55K-deleted oncolytic Ads cannot replicate in normal cells. Permissive cancer cells provide E1B55K-like factors to relax cyclin E regulation and allow E1b55K-deleted Ads to induce cyclin E expression; leading to preferential oncolytic replication. 


\subsection{Scope of the dissertation}

Ad d/1520 was originally created in United States, but the development for clinical applications was aborted. However, this vector with a slight modification became the first approved oncolyitc virus product $(\mathrm{H} 101)$ on the China market and now achieves some measure of success in cancer treatment (18). There is an urgent need for us to improve current strategies to design viral vectors, and further improve the insights into the field of oncolytic virotherapy. The work contained within this dissertation has been proposed based on the hypothesis that cyclin E deregulation in cancer cells is the molecular basis of oncolytic selectivity of E1b55K-deleted Ads. We advanced the previous findings to further develop the mechanism model to demonstrate the role of cyclin $E$ in the selective replication of E1b55K-deleted oncolytic Ads. The virtual application was then derived from the knowledge of this mechanism model. Our laboratory then constructed a novel tumor-specific oncolytic Ad-cycE with cyclin E promoter to drive E1A expression. The oncolytic efficacy of this novel oncolytic Ad vector has been evaluated in vitro and in vivo. Additional work has been completed exploring the impact of a combination treatment with $\mathrm{Ad}-\mathrm{cycE}$ and rapamycin which induces rapamycin in lung cancer. Ultimately, by uncovering the selective replication mechanism of oncolytic Ad and its further application, we hope to overcome the current limitations; leading to advancement for the field of oncolytic virotherapy. 


\section{CHAPTER II}

\section{MOLECULAR BASIS FOR VIRAL SELECTIVE REPLICATION IN CANCER CELLS: ACTIVATION OF CDK2 BY ADENOVIRUS-INDUCED CYCLIN E}

\subsection{Introduction}

Human adenoviruses (Ads) are double-stranded linear DNA viruses that are able to infect and replicate in a wide variety of cell types in vitro and in vivo, including post-mitotic cells. After infection, viral early proteins interact with cellular factors to create favorable environments for viral replication (37). The Ad E1 region contains two sets of genes, E1a and E1b, that are dedicated to cell cycle control, apoptotic inhibition, and cellular and viral gene regulation (97). Ads with $E 1$ modifications that preferentially replicate in cancer cells have been used for cancer gene therapy (15-17).

The viral E1a gene is expressed immediately after infection. The primary role of E1a gene products is to regulate expression of multiple cellular and viral genes (37). Instead of directly binding to specific DNA sequences in transcriptional regulation elements, E1A proteins interact with several key regulators of cell proliferation $(98,99)$. The well-known cellular factors to which E1A proteins bind are products of the retinoblastoma $(R b)$ gene and its structurally related genes, $p 107$ and $p 130(100,101)$. By sequestering the retinoblastoma protein $(\mathrm{pRb}), \mathrm{E} 1 \mathrm{~A}$ 
activates transcriptional regulator E2F proteins. Studies have suggested that the pRb/E2F complex actively represses transcription from target genes and mediates $\mathrm{G}_{1}$ arrest triggered by p19 (ARF), p53, p16INK4a, TGF beta, or cell contact (102-104). Recently Pelka et al. (2011) indicated that E1A can directly bind to E2F/DP complexes by interacting with DP-1, resulting in the activation of E2F-responsive gene expression independently of binding to $\mathrm{pRb}$ (105). Several groups have shown that expression of E1a gene triggers the accumulation of p53 protein and p53-dependent apoptosis $(40,106)$ either by activating p53 transcription or preventing p53 from being degraded by the proteasome $(40,41$, 43, 106).

Ad E1B55K has been shown in some studies to counteract the E1A-induced stabilization of p53 $(106,107)$. E1B55K protein may inhibit the functions of p53 through at least three distinct mechanisms. E1B55K reportedly binds the amino terminus of p53 (44), and this binding may repress p53 transcriptional activation, as suggested in transcription assays (45) and transient transfection studies (108). E1B55K may also interfere with p53 function by cooperating with viral E4orf6 protein to cause proteolytic degradation of p53 protein (46-48). A recent study has shown that E1B55K alone functions as an E3 SUMO1-p53 ligase that interacts with promyelocytic leukemia nuclear bodies to inactivate p53 and stimulate its nuclear export (49). Thereby, E1B55K blocks the expression of p53-regulated genes and, consequently, counteracts the p53-dependent apoptosis induced by E1A; allowing efficient viral replication $(44,45)$. 
Ad d/1520 (ONYX-015) contains an 827-bp deletion and a point mutation generating a premature stop codon in the E1B55K coding sequence, preventing expression from the gene (15). It was originally proposed that the $E 1 b 55 K$-deleted Ads could replicate only in p53-deficient tumor cells, as the E1B55K-mediated degradation of p53 protein was not required in those cancer cells $(16,109)$. E1b55K-deleted oncolytic Ads have been tested in human clinical trials and are being marketed for cancer treatment in China after approved by China's State Food and Drug Administration (SFDA) (18). However, the original hypothesis was challenged by several studies showing that E1b55K-deleted Ads are able to replicate in cells regardless of their p53 status (52-55). Further studies have shown that the accumulation of p53 protein, after infection with Ads carrying mutated $E 1 b 55 K$ genes that are unable to repress p53, can neither efficiently induce apoptosis nor transcriptionally activate expression of p53-responsive genes in Ad-infected cells $(62,63)$. Thus, these results suggest that blocking of p53 activity by E1B55K protein is unlikely to be the major requirement for viral replication. The mechanism(s) of E1b55K-deleted viral replication in cancer cells is still not established, even though the vectors have already been applied in the clinic for human cancer treatment (18).

Previously, we have shown that Ad E1B55K is involved in the induction of cell cycle-related genes, including cyclin E and CDC25A (79). Ad E1B55K mediates the large form of cyclin E protein (cyclin EL) induction in Ad-infected cells (56). Cyclin $E$ and the large form cyclin EL are generated from the alternative splicing. The translation of cyclin EL is initiated at an ATG codon located in exon 2 and 
cyclin $E$ is from the ATG codon in exon 3 (80). The E1B55K function is required for cyclin EL induction in normal cells, but is not required in cancer cells with deregulated cyclin E. Failing to efficiently induce cyclin EL expression in the normal cells, replication of E1b55K-deleted oncolytic Ads is restricted. However, E1b55K-deleted oncolytic Ads can efficiently induce cyclin EL in cancer cells and carry out sufficient oncolytic replication. We proposed that cyclin E deregulation in cancer cells may be an important molecular basis for the selective oncolytic replication of E1b55K-deleted Ads (56).

Cyclin E regulates cell cycle progression, DNA replication $(110,111)$, and centrosome duplication $(112,113)$. Expression of cyclin $E$ is strictly controlled in normal cells. The level of cyclin $E$ rises at late $G_{1}$ phase, peaks at the $G_{1} / S$ phase to promote the S-phase entry, and decreases thereafter $(80,81)$. Deregulation of cyclin $E$ is frequently detected in many types of cancers, as cyclin $E$ gene amplification (91), overexpression of cyclin E mRNA or protein levels $(87,92)$, decrease of cyclin E turnover (93), and the presence of more active forms of cyclin E (94-96). Constitutive overexpression of cyclin E was shown to induce chromosome instability and impair normal cell cycle progression $(89,90)$. The hypothesis that abnormal cyclin E expression can trigger tumors has also been supported by transgenic animal studies $(86,114,115)$.

One function of cyclin $\mathrm{E}$ is to bind and activate cyclin-dependent kinase 2 (CDK2) (82). The cyclin E/CDK2 complex then phosphorylates substrates such as $\mathrm{pRb}$ and leads to transcriptional activation of downstream genes. Studies also indicate 
that cyclin E has CDK2-independent functions $(83,84)$. In vivo animal studies indicate variance between the phenotypes of cyclin $\mathrm{E}$ null (cyclin $\mathrm{E}^{-/-} \mathrm{E}^{-/}$) mice and CDK2 null $\left(\mathrm{CDK}^{-1-}\right)$ mice. Mice lacking CDK2 are viable, with normal development except defective germ cell development $(116,117)$; yet knockout of cyclin E1 and E2 genes in mice causes embryonic lethality owing to the deficiency in endoreplication of trophoblast giant cells and megakaryocytes (118). Matsumoto et al. (2004) identified a centrosomal localization signal (CLS) domain in cyclin $E$ (119). This CLS domain allows cyclin E to target the centrosome and promote S phase entry in a CDK2-independent manner. Additionally, Geng et al. (2007) showed that a cyclin E kinase-deficient mutant (KD-E) is able to partially restore minichromosome maintenance protein (MCM) loading and S phase entry in cyclin E null cells (83). Thus, cyclin E has CDK2-dependent and independent functions in S phase entry and DNA replication. An important question is whether Ad-induced cyclin E may activate CDK2 and whether the cyclin E-CDK2 interaction may play a crucial role in Ad replication. This question is especially important in the development of oncolytic virotherapy strategies.

We report here that Ad-induced cyclin E binds with and activates CDK2 that targets transcription repressor $\mathrm{pRb}$, which in turn can regulate expression of cellular and viral genes. The results suggest that the interaction between the Ad-induced cyclin E and CDK2 is to generate a suitable environment for Ad productive replication. 


\subsection{Materials and Methods}

Cell lines and culture conditions. HEK 293 (ATCC no. CRL-1573), human lung fibroblast WI-38 (ATCC no. CCL-75), and human lung cancer A549 (ATCC no. CCL-185) cell lines were purchased from the American Type Culture Collection (Rockville, MD). WI-38 cells were cultured in minimal essential medium (MEM) Alpha GlutaMAX with $0.1 \mathrm{mM}$ non-essential amino acids and $1.0 \mathrm{mM}$ sodium pyruvate. HEK 293 and A549 cells were cultured in minimal essential medium Alpha. All media were supplemented with $10 \%$ fetal bovine serum (FBS) and penicillin/streptomycin $(100 \mathrm{U} / \mathrm{ml})$. Cells were cultured in a $5 \% \mathrm{CO}_{2}$ incubator at $37^{\circ} \mathrm{C}$. All cell culture reagents were obtained from Gibco BRL (Bethesda, MD).

Adenoviral vectors. Wild-type adenovirus type 5 (Adwt, ATCC no. VR-5) was used as a replication-competent control. AdCMV/GFP, an Ad vector with E1 deletion carrying a green fluorescent protein (GFP), was used as a replication-defective control. Adhz63, an oncolytic Ad vector with the deletion of E1b55K region, was constructed by our laboratory (38).

Viral infection and titration. Cells were seeded into 6-well plates at a density of $2.5 \times 10^{5}$ (cells/well) and cultured under the indicated conditions. Subsequently, cells were mock-infected or infected with AdGFP, Adwt, or Adhz63 at a multiplicity of infection (MOI) of 5. Cytopathic effect (CPE) was observed at designed time points and photographed with an inverted microscope (Olympus CKX41). Total infected cells and culture supernatants were collected at $48 \mathrm{hr}$ postinfection (p.i.) 
and lysed to release virus particles with three cycles of freezing and thawing. The viral titers were determined by the infective unit method as described previously $(120,121)$. Briefly, HEK 293 cells were seeded in 96-well plates at a density of $10^{3}$ (cells/well) and then infected with 5 -fold serially diluted viruses. CPE was recorded and scored after incubation for 7 days. The reduction percentage in virus titer is calculated by the formula, reduction $\%=[($ titer of control group - titer of experimental group) / titer of control group] x $100 \%$.

Viral DNA synthesis assay. After viral infection, cells were collected at different time points. The viral DNA synthesis was determined with Southern blot; $1 \mu \mathrm{g}$ of isolated genomic DNA was digested with the restriction enzyme Pstl and analyzed with $1 \%$ agarose gel, which was subsequently transblotted to a Hybond-N+ membrane (YA3609; Amersham Pharmacia Biotech, Arlington Heights, IL). The probe was prepared by digesting $0.5 \mu \mathrm{g}$ pBHGE3 (122) with Pstl and labeled by following the protocol of Amersham AlkPhos Direct Labeling and Detection Systems (RPN 3690; GE Healthcare, Piscataway, NJ). The blot was prehybridized for $3 \mathrm{hrs}$ at $63^{\circ} \mathrm{C}$. The hybridization and stringency washes were performed at $55^{\circ} \mathrm{C}$ and followed by the chemiluminescent detection according to the manufacturer's protocol.

Western blot analysis. Infected cells were harvested at indicated time points and lysed with CDK2 lysis buffer (20 mM Tris $\mathrm{pH} 7.5,150 \mathrm{mM} \mathrm{NaCl}, 5 \mathrm{mM} \mathrm{MgCl}$, 0.5\% Nonidet P-40, 0.1\% Brij 35, $5 \mathrm{mM}$ sodium glycerophosphate, $1 \mathrm{mM}$ sodium 
vanadate, $1 \mathrm{mM}$ dithiothreitol). The Western blot analyses were performed as described previously (123). Briefly, $80 \mu \mathrm{g}$ of cell lysates were electrophoresed through $12 \%$ SDS-polyacrylamide gels and transferred onto an Immobilon-P Membrane (Millipore, Billerica, MA). The primary antibodies used in this study were rabbit anti-cyclin E (M-20), CDK4 (C-22), mouse anti-cyclin D1 (DCS-6), PCNA (PC10), p21 (F-5), pRb (IF8) (Santa Cruz Biotechnology, Santa Cruz, CA), mouse anti-CDK2, p27 (BD Biosciences, San Jose, CA), pCDK2 T160 (Cell signaling, Danvers, MA), rabbit anti-phosphorylated pRb (phospho-pRb) S612, and actin (Sigma, St. Louis, MO), anti-phospho-pRb S795 (New England Biolabs, Beverly, MA), and anti-phospho-pRb T821 (Invitrogen, Carlsbad, CA). Actin was used as an internal control. The membranes were then incubated with anti-mouse immunoglobulin $G$ ( $\lg G$ ) or anti-rabbit IgG peroxidase-linked species-specific whole antibody (GE Healthcare, Piscataway, NJ). Chemiluminescent detection was performed with ECL reagents according to the supplier's recommendations (GE Healthcare). The scanned band intensity was quantitated by Gel-pro Analyzer 4.0 software (Media Cybernetics, Bethesda, MD) according to the manufacturer's tutorial. Densitometric value for each band was expressed as integrated optical density (I.O.D.) and the results were normalized with actin. The final values represent the means of relative percentage change, from at least three independent experiments, compared with the mock group \pm S.D.. Statistical difference was assessed with Student's $t$-test. A p-value of $<0.05$ was considered significant. 
Immunoprecipitation. A549 cells were seeded in $150 \mathrm{~mm}$ dishes at a cell density of $5 \times 10^{6}$ (cells/dish) and then mock-infected or infected with AdGFP, Adwt, or Adhz63 at an $\mathrm{MOI}$ of 5 . At $48 \mathrm{hr}$ p.i., cells were collected and lysed with CDK2 lysis buffer according to the method described in previous publications $(56,124)$. Cell lysates $(500 \mu \mathrm{g})$ were immunoprecipitated with cyclin E (HE111), the mouse monoclonal antibody (Santa Cruz), or anti-CDK2 antibody (BD Transduction Laboratories) at $4^{\circ} \mathrm{C}$ for $4 \mathrm{hr}$, followed by adding protein $\mathrm{A}$ Sepharose $\mathrm{CL}-4 \mathrm{~B}$ (82506; Sigma) and incubating overnight. Immunocomplexes were analyzed by Western blot with anti-cyclin E and CDK2 antibodies.

Small interfering RNA (siRNA) transfection. The siRNA oligonucleotides were synthesized by Eurogentec (Fremont, CA). Three different siRNA duplexes were designed to target CDK2 on nucleotides 399 to 419 (\#1), 619 to 639 (\#2), and 691 to 711 (\#3) according to Genbank accession NM001798.2 (National Center for Biotechnology Information GenBank). A negative control siRNA duplex containing two strands of 19 complementary RNA bases with 3'dTdT overhangs was obtained from Eurogentec (SR-CL000-005). Cells were seeded into a 6-well plate at a density of $10^{5}$ (cells/well) and then transfected with $200 \mathrm{nM}$ CDK2 siRNA duplexes or a non-specific control siRNA duplex with Lipofectamine 2000 (Invitrogen, Carlsbad, CA) according to the manufacturer's protocol. Cells were harvested at $48 \mathrm{hr}$ after transfection. Eighty $\mu \mathrm{g}$ of cell lysates were analyzed by Western blot with CDK2, pCDK2 T160, pRb, phosphorylated pRb (p-pRb), cyclin E, capsid proteins, and actin antibodies. 


\subsection{Results}

Cyclin E/CDK2 complex formed in cells infected with adenoviruses. We have previously established the link between cyclin E and replication of adenoviruses $(56,79)$. The data shown in Figure 2.1 recapitulate that Ad E1B55K participates in the induction of the large form of cyclin E protein (cyclin EL), which contributes to the efficient viral replication. Cyclin E and cyclin EL are generated from alternative splicing with different start ATG codons in exons 2 and 3 (80). The $\mathrm{N}$ terminus of virus-induced cyclin EL is 15 amino acids longer than that of cyclin E protein. Cyclin E protein is constitutively expressed in A549 human lung cancer cells (56). Wild-type Ad5 (Adwt), with the intact E1b55K region, induced significant cyclin EL expression in both of WI-38 human lung fibroblast cells and A549 human lung cancer cells (Fig. 2.1A, lanes 2 and 5), and caused efficient cytopathic effect (CPE) (Fig. 2.1B, panels b and e). Ad vector Adhz63 with E1b55K-deletion (38) also induced significant cyclin EL in A549 cells (Fig. 2.1A, lane 6) and caused efficient CPE of the cells (Fig. 2.1B, panel c). However, the vector failed to induce cyclin EL overexpression in WI-38 cells (Fig. 2.1A, lane 3) and their CPE (Fig. 2.1B, panel f). To compare the replication of Adwt and Adhz63 in A549 and WI-38 cells, the titers of these two viruses were determined. The Adhz63 replication was strongly repressed in WI-38 cells, showing only $10 \%$ of relative replication in comparison with Adwt (Fig. 2.1C). When we compared Adhz63 replication with that of Adwt in A549 cells, consistent with the CPE results, $80 \%$ of relative replication of Adhz63 was observed. Thus, Adhz63 replication is more repressed in WI-38 cells than in A549 cells. The result is consistent with our previous 
observation that cyclin EL induction in cancer cells is connected with the selective replication of $E 1 b 55 K$-deleted Ads in cancer cells (56). 
A

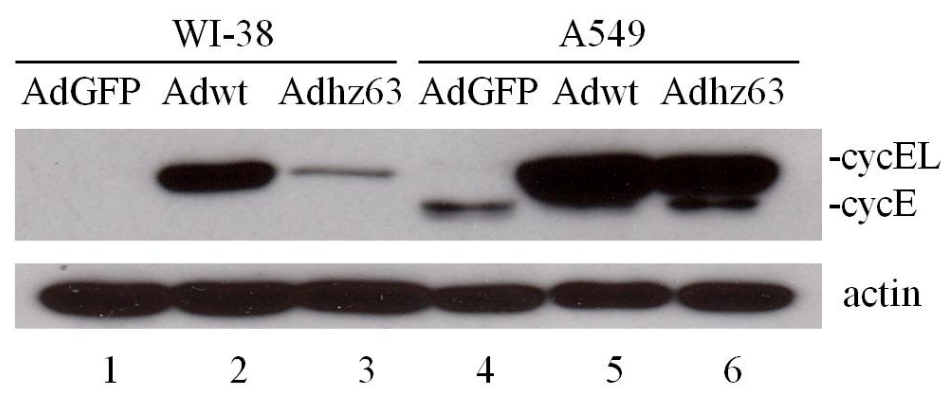

B

AdGFP

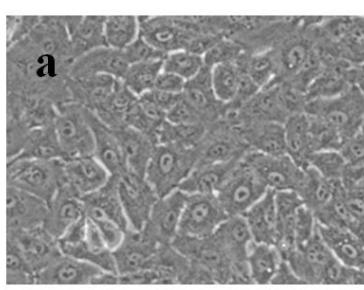

A549

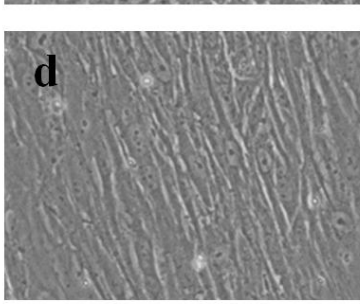

Adwt
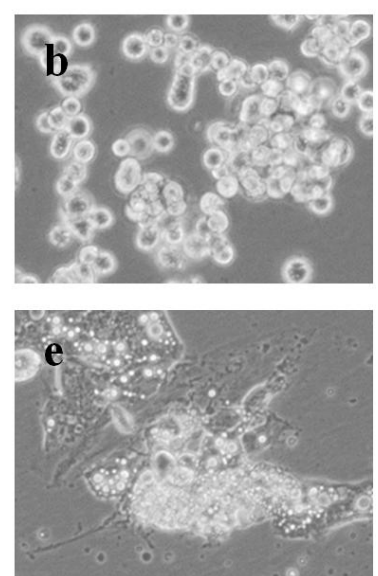

Adhz63
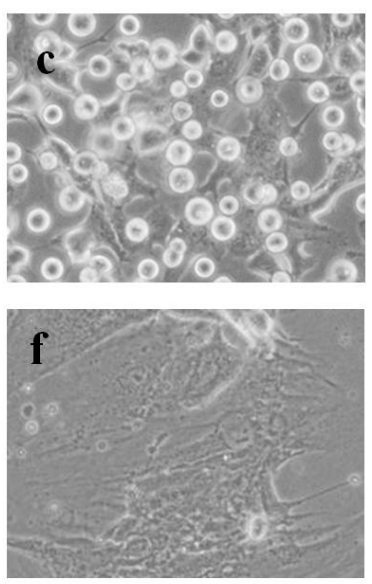

C
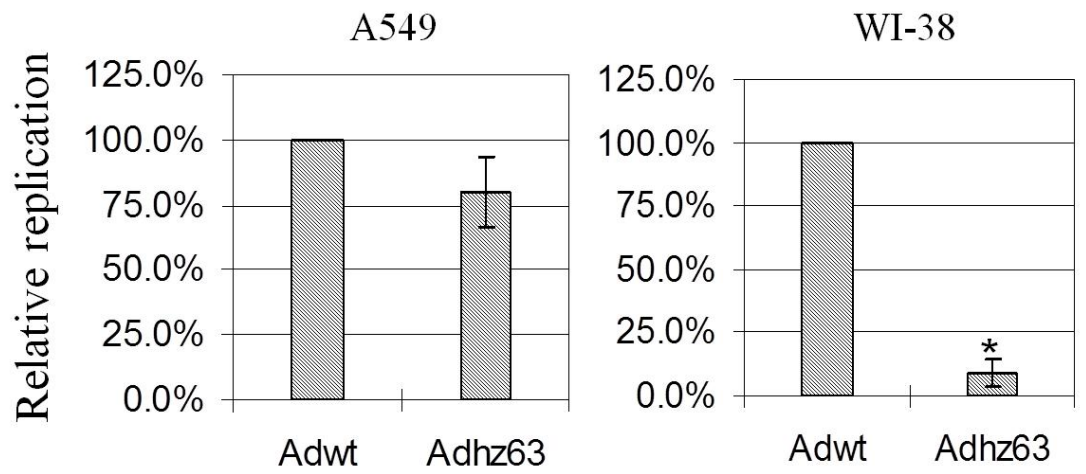
Figure 2.1. Virus replication is correlated with cyclin E overexpression. WI-38 or A549 cells were infected with AdGFP, Adwt or Adhz63 at an MOI of 5. (A) Cells were collected at $48 \mathrm{hr}$ and then analyzed by Western blot. Cell lysates were immunoblotted for cyclin E and actin. Actin was used as a loading control. (B) CPE was photographed at $72 \mathrm{hr}$ post infection (p.i.). All microscopy was originally at a magnification of $\times 100$. (C) Viral titers were determined at $72 \mathrm{hr}$ p.i. with the infection unit method. The value indicates the mean of three independent experiments, shown as the mean change percentage relative to Adwt control group \pm S.D. ${ }^{*} \mathrm{P}<0.05$ compared with the Adwt group, Student's $t$-test. 
Cyclin E can promote the S phase entry and participate in DNA replication via CDK2-dependent (82) and CDK2-independent pathways (119). To study whether cyclin E function in Ad replication is CDK2 dependent or independent, we first sought to investigate the physical contact between CDK2 and cyclin EL in cells affected by Ads. Lung cancer A549 cells were mock-infected or infected with AdGFP, Adwt, or Adhz63. To understand how E1b-deleted Ads selectively replicate in cancer cells, we focused on A549 lung cancer cells in which both Adwt and the E1b-deleted Adhz63 can efficiently induce cyclin EL and replicate. At $48 \mathrm{hr}$ post-infection (p.i.), cells were collected and lysed. We first used anti-cyclin E antibody to immunoprecipitate cyclin E protein and analyzed the immunocomplexes with Western blot. The data show that cyclin E protein precipitated from cells mock-treated or treated with replication-defective AdGFP (negative controls) did not exhibit significant association with CDK2 protein (Fig. 2.2A, lanes 1 and 2). However, immunocomplexes from Adwt- and Adhz63-infected A549 cells contained both cyclin E and cyclin EL with an increase of CDK2 binding (Fig. 2.2A, lanes 3 and 4). To verify this cyclin E/CDK2 bonding, we also used anti-CDK2 antibody to pull down the protein complex and then examined the level of cyclin E proteins in the cyclin E-CDK2 complex. The immunoprecipitated CDK2 protein was increased in Adwt and Adhz63-infected cells with a concomitant precipitation of cyclin EL (Fig. 2.2B, lanes 3 and 4), especially for Adwt-infected cells (lane 3). The results show that replication-competent Adwt and Adhz63 induce cyclin EL expression and increase the formation of cyclin EL/CDK2 complex in A549 cancer cells, indicating that the 
cyclin EL induced in Ad-infected cells strongly associates with CDK2. 


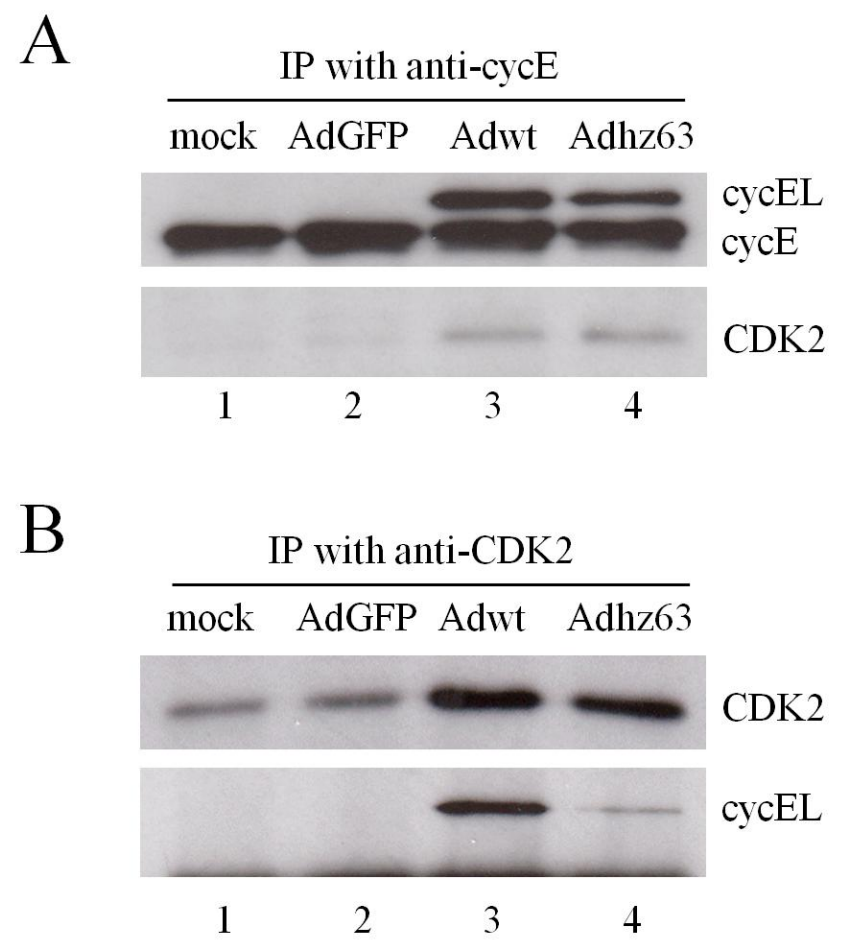

Figure 2.2. Cyclin E/CDK2 complex induced by viral infection in A549 cells.

(A) A549 cell lysates were immunoprecipitated with anti-cyclin E antibody (1:50 dilution). Immunocomplexes were analyzed by Western blot with cyclin $E$ and CDK2 antibodies. (B) The cell lysates were immunoprecipitated with anti-CDK2 antibody and immunoblotted for CDK2, cyclin E and cyclin EL. 
Adenovirus-induced cyclin EL increases CDK2 phosphorylation. CDK2 is activated by the phosphorylation at the T160 site and this phosphorylation increases its electrophoretic mobility, resulting in faster-migrating bands (125). We investigated whether cyclin EL induction and the increased interaction between cyclin EL and CDK2 in A549 cells after Adwt and Adhz63 infection may promote CDK2 phosphorylation at the specific T160 site. Analysis of the cell lysates with Western blot demonstrated that the cyclin EL induction led to an increase of the faster-migrating CDK2, consistent with phosphorylated-CDK2 protein (pCDK2) T160 (the active form of CDK2), especially at $48 \mathrm{hr}$ p.i. (Fig. 2.3A, lanes 7 and 8). We verified the faster-migrating form of CDK2 with phospho-CDK2 (T160) antibody (\#2561, Cell signaling). Densitometric analysis of these bands demonstrated that Adwt infection caused a 1.3 to 2.7 -fold increase $(P=0.03)$ in the level of pCDK2 T160 and Adhz63 infection caused a 1.5 to 1.9-fold increase $(P=0.0026)$ compared with the mock-control group at $48 \mathrm{hr}$ p.i. (Fig. 2.3B, lanes 3 and 4). The result in Figure 2.3B is consistent with that in Figure 2.3A (lanes 7 and 8).

In addition to cyclin $E$, cyclin $D$ is also involved in the transition of the $G_{1}-S$ phase. Thus, we also examined the level of cyclin D. Interestingly, the level of cyclin D was decreased after viral infection. Densitometric analysis of the bands demonstrated that Adwt and Adhz63 infection at $24 \mathrm{hr}$ decreased cyclin D protein to the levels of $11 \%(P=0.0000025)$ and $71 \%(P=0.001)$ of the mock infection, respectively (Fig. 2.3C, lanes 3 and 4). The levels of cyclin D in cells infected with 
Adwt or Adhz63 were further decreased at $48 \mathrm{hr}$ to $3 \%(P=0.00000043)$ and $8 \%$ $(P=0.00002)$, respectively (Fig. $2.3 \mathrm{C}$, lanes 7 and 8$)$. Meanwhile, the level of CDK4 and the proliferating cell nuclear antigen (PCNA) did not significantly change in any of the groups. CDK4 is regulated and activated by cyclin D to process the $\mathrm{G}_{1}-\mathrm{S}$ transition $(126,127)$; PCNA, known to regulate DNA replication and DNA repair, is associated with multiple cyclin/CDK complexes in the cell-cycle progression $(128,129)$. The results show that Ads decreased cyclin D production and did not affect the levels of CDK4. Thus, Ad infection specifically induced cyclin EL that activated CDK2 via phosphorylation at its T160 site, suggesting a critical role of cyclin EL and CDK2 in Ad replication. 
A

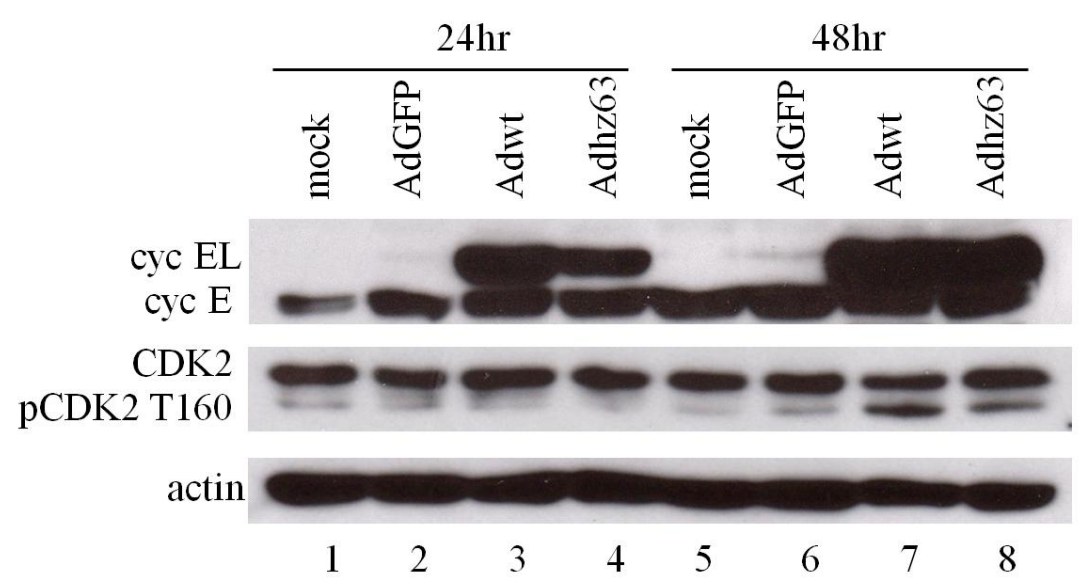

B

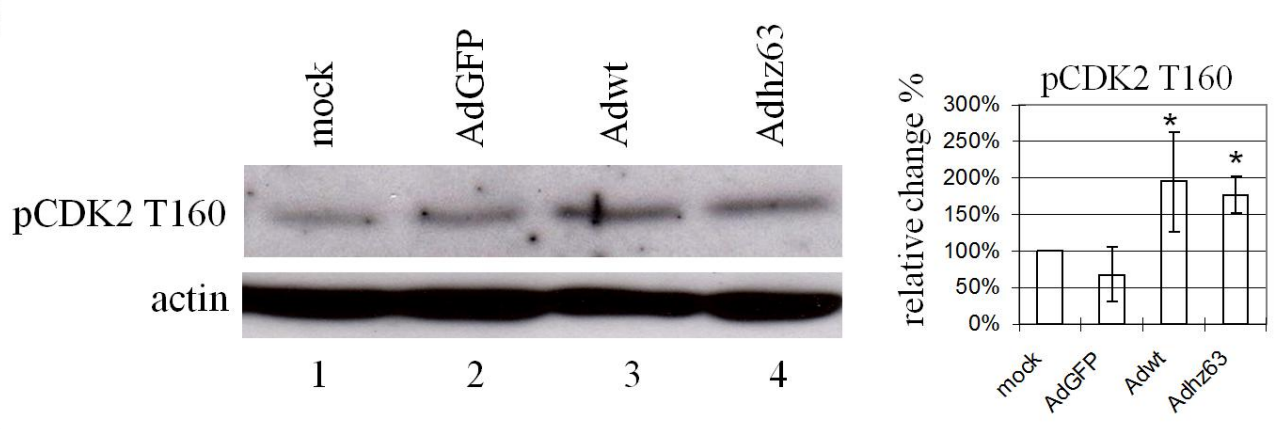

C

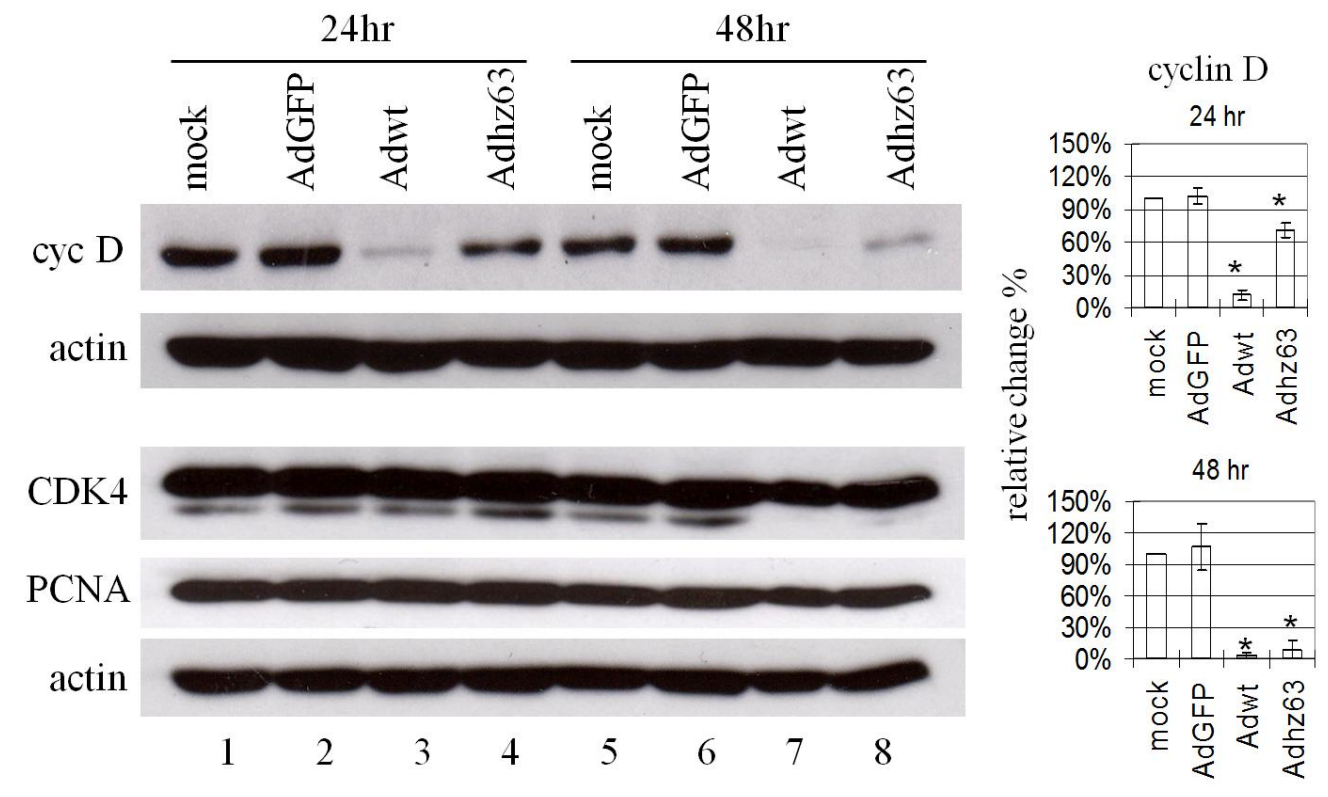


Figure 2.3. Effects of viral replication on cellular proteins related to $G_{1} / S$ phase. A549 cells were mock infected or infected with AdGFP, Adwt, or Adhz63 at an $\mathrm{MOI}$ of 5 . Cells were collected at $24 \mathrm{hr}$ or $48 \mathrm{hr}$ p.i. and then analyzed by Western blot. Cell lysates were immunoblotted for (A) cyclin E and CDK2; (B) pCDK2 T160; and (C) cyclin D, CDK4 and PCNA. Actin was used as a loading control. The scanned band intensity was quantitated and the values represent the means of the relative change percentages compared with the mock group \pm S.D. from three independent triplicate experiments. * $\mathrm{P}<0.05$ compared with the mock-control group, Student's $t$-test. 
Adenoviruses increase pRb phosphorylation. Cyclin E-activated phosphorylated CDK2 (pCDK2) is known to control the $G_{1}-S$ transition by phosphorylation of the downstream substrates. Considering that $\mathrm{pRb}$ is one of the well-known targets for pCDK2, we examined whether the increase of activated pCDK2 alters the phosphorylation of $\mathrm{pRb}$ at $\mathrm{S612}$, which is a CDK2-preferred phosphorylation residue $(130,131)$. We found that the level of phospho-pRb S612 was increased to $314 \%(P=0.016)$ and $240 \%(P=0.03)$ in cells infected with Adwt and Adhz63, respectively, even though the protein level of unphosphorylated pRb is decreased slightly (Fig. 2.4A, lanes 3 and 4). We could not detect any significant changes of $\mathrm{p}-\mathrm{pRb}$ T821, another CDK2-preferred phosphorylation residue $(130,132)$, and the CDK4-preferred p-pRb S795 (133) (Fig. 2.4A). The results suggest the selection of the $\mathrm{S} 612$ site in $\mathrm{pRb}$ by Ad-activated CDK2 for protein phosphorylation.

Adenoviruses repress CDK inhibitors. We also observed that the protein levels of both p21 and p27 are decreased in A549 cells infected with Adwt and Adhz63, especially for p21 (Fig 2.4B). p21 and p27 are the well-known CDK inhibitors, which negatively regulate the activity of cyclin E/CDK2 complexes to prevent the cell-cycle progression (134). Densitometric analysis of these bands demonstrated that the level of p21 protein decreased to the levels of $8 \%(P=0.0000002)$ and 44\% ( $P=0.00066)$ of the mock control in A549 cells after infection with Adwt and Adhz63 at $24 \mathrm{hr}$, respectively (Fig. 2.4B, lanes 3 and 4). The p21 level was further repressed in A549 cells at $48 \mathrm{hr}$ after infection with Adwt $(2 \%, \mathrm{P}=0.00000034)$ 
and Adhz63 (6\%, $\mathrm{P}=0.0000007)$ (Fig. 2.4B, lanes 7 and 8). Ad infection also decreased p27 protein levels to 36\% (Adwt, $\mathrm{P}=0.0015)$ and 49\% (Adhz63, $\mathrm{P}=$ $0.00043)$ at $24 \mathrm{hr} ; 16 \%($ Adwt, $P=0.00012)$ and $37 \%($ Adhz63, $P=0.0092)$ at 48 hr in A549 cells (Fig. 2.4B). The results suggested that Ads activate the CDK2 by inducing cyclin EL and repressing p21 and p27. 
A

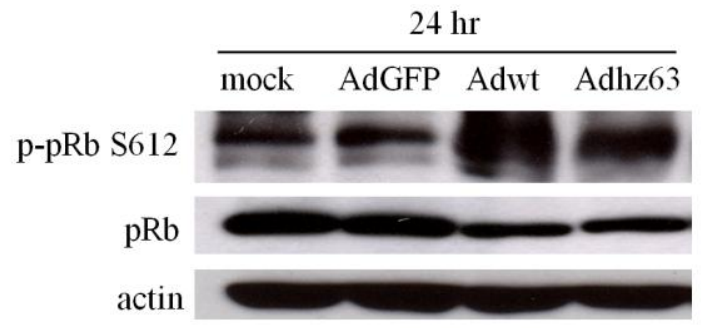

p-pRb T821

actin
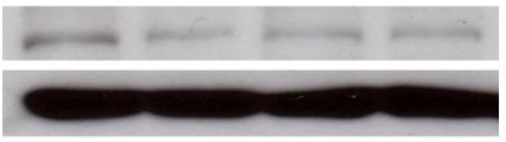

p-pRb S795

actin

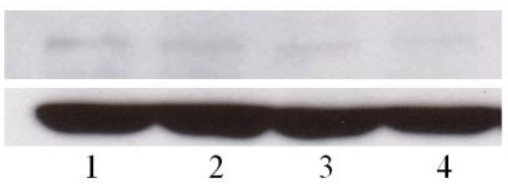

B

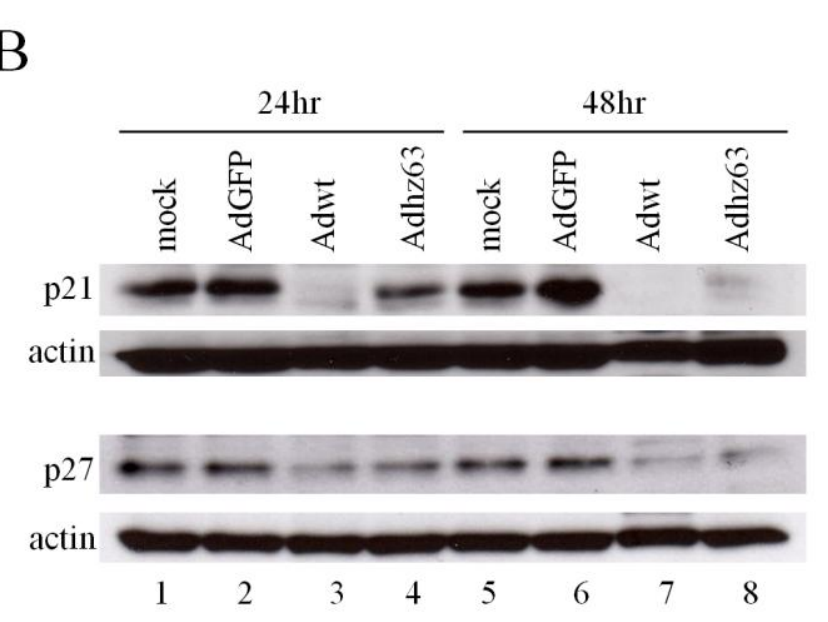

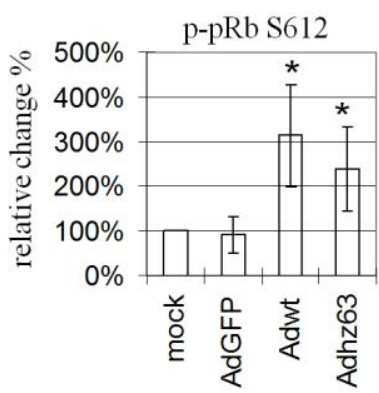<smiles>C1CCC1</smiles>

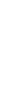

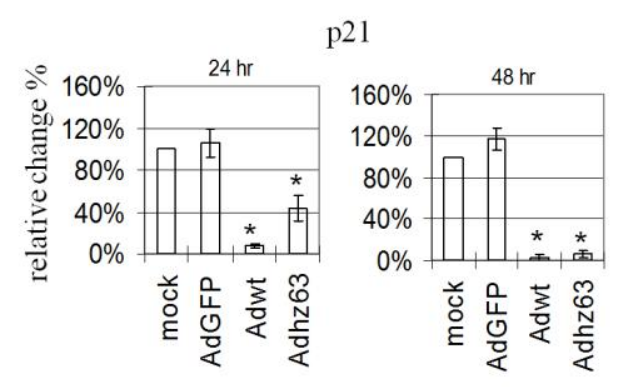

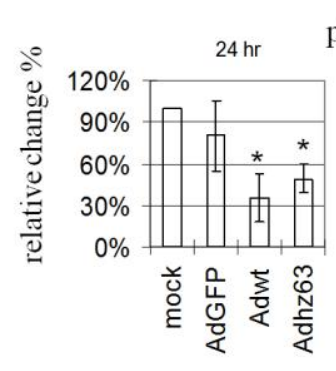

p27

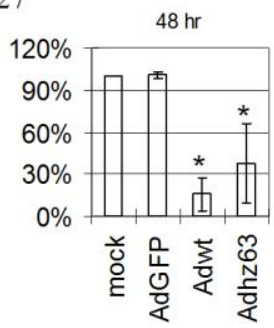


Figure 2.4. Effects of viral replication on pRb and CDK inhibitors. A549 cells were mock-infected or infected with AdGFP, Adwt, or Adhz63 and collected at 24 hr or $48 \mathrm{hr}$ p.i., followed by Western blot analysis. Cell lysates were immunoblotted for (A) pRb, phospho-pRb (p-pRb) at S612, T821 and S795 or (B) p21 and p27. Actin was used as a loading control. * $\mathrm{P}<0.05$ compared with the mock-control group, Student's $t$-test. 


\section{Interruption of cyclin EL and CDK2 interaction reduces adenoviral}

replication. To further investigate the role of CDK2 in viral replication, we used the CDK2 chemical inhibitor roscovitine (Ros; CYC202) to interrupt cyclin EL and CDK2 interaction. Ros is a purine derivative that inhibits the activity of CDK2 by binding to its active site (135). Ros reduces phosphorylation on CDK2 (136) and blocks the androstenedione-induced increase of active phosphorylated CDK2 (137). If activation of CDK2 is required for viral replication, blocking CDK2 activity should reduce it. Figure $2.5 \mathrm{~A}$, representing one of the four repeated experiments, shows that with increased Ros, CPE caused by Adwt and Adhz63 infection was partially inhibited. Figure $2.5 \mathrm{~B}$ shows that treatment with $5 \mu \mathrm{M}$ of Ros led to a $50 \%$ reduction in Adwt titer $(P=0.0002)$ and $71 \%$ reduction in Adhz63 titer $(P=0.034)$ when compared with the vehicle-control group treated with dimethyl sulfoxide (DMSO, defined as $0 \mu \mathrm{M}$ Ros). Treatment with $10 \mu \mathrm{M}$ Ros led to more decreases

of viral titers: $81 \%$ reduction in Adwt $(P=0.00001)$ and $87 \%$ reduction in Adhz63 $(P=0.012)$. The repressed viral yields are consistent with the CPE phenomenon in Figure 2.5A. 


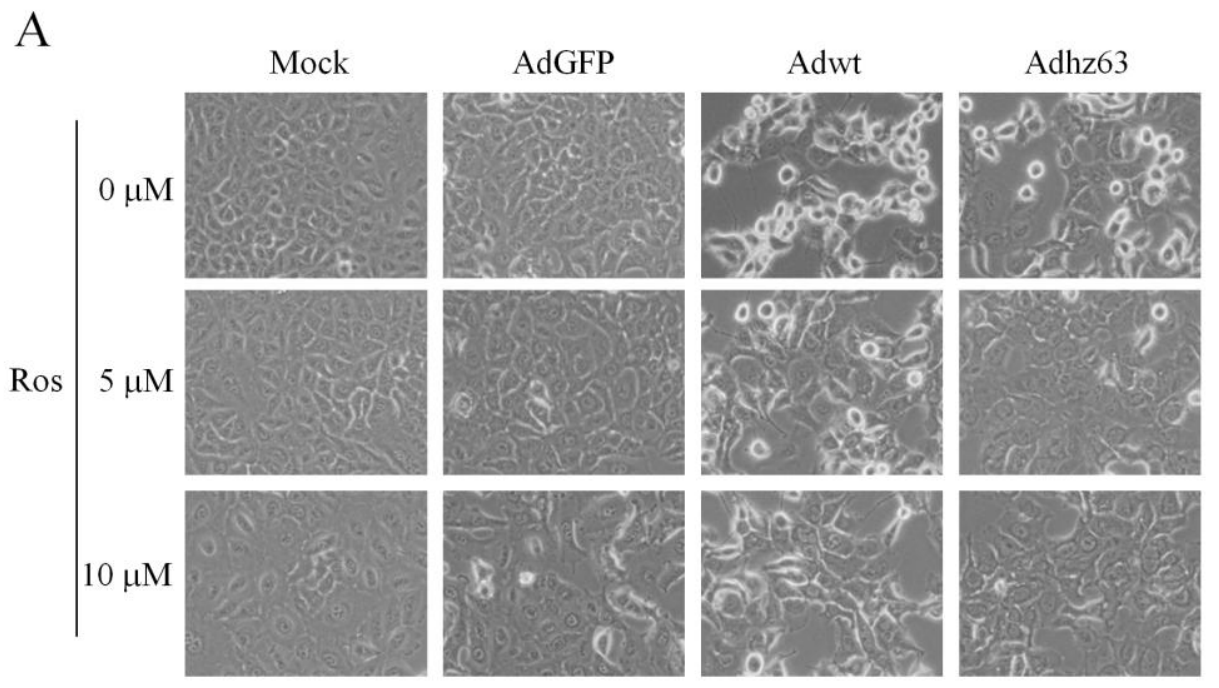

B

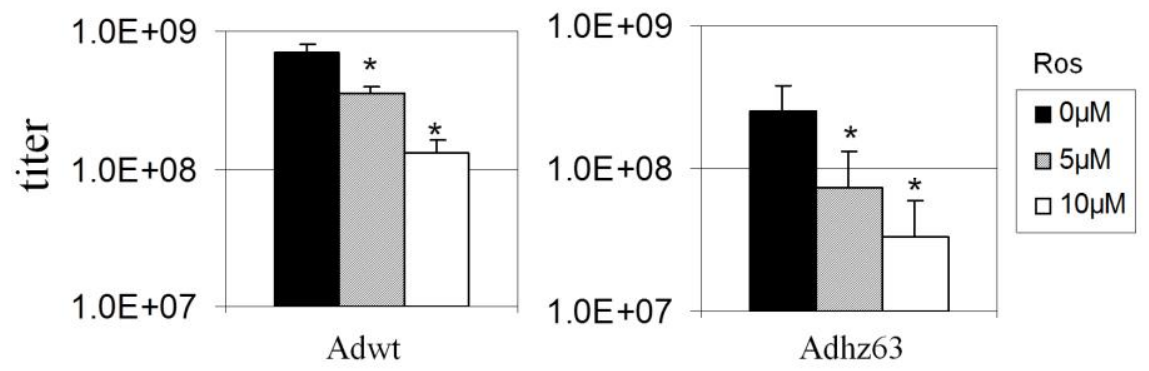

Figure 2.5. Effects of roscovitine on CPE and viral production. (A) Cells were treated with $0 \mu \mathrm{M}$ (vehicle-control group treated with DMSO), $5 \mu \mathrm{M}$, or $10 \mu \mathrm{M}$ roscovitine, and mock-infected or infected with AdGFP, Adwt or Adhz63 at an MOI of 5 . All microscopy is originally at a magnification of $x 100$ taken at $48 \mathrm{hr}$ p.i. (B) Viral titers were determined at $48 \mathrm{hr}$ p.i. with the infection unit method. The values represent the means \pm S.D. of independent quadruplicate. ${ }^{*} \mathrm{P}<0.05$ compared with the $0 \mu \mathrm{M}$ roscovitine, Student's $t$-test. 
We then examined the levels of viral DNA and proteins produced in cells affected by Ros treatment. The viral DNA synthesis was determined by Southern blot probed with the Ad genome. The linear Adwt DNA is $36 \mathrm{~Kb}$ with total 28 Pstl restriction sites. The largest fragment is $4333 \mathrm{bp}$ and the smallest fragment is only $12 \mathrm{bp}$. The sizes of the representative DNA fragments were marked on Figure 2.6. The viral DNA synthesis of Adwt and Adhz63 at $24 \mathrm{hr}$ p.i. was strongly inhibited in the presence of $10 \mu \mathrm{M}$ Ros (Fig. 2.6A, lanes 6 and 12). Consistently, the viral capsid proteins were significantly inhibited in the presence of $10 \mu \mathrm{M}$ Ros (Fig. 2.6B, lanes 4 and 6). Inhibition of CDK2 activity with Ros reduced the phosphorylation of pRb at the S612 site in AdGFP, Adwt and Adhz63-treated cells (Fig. 2.6C). Interestingly, Ros treatment markedly repressed the induction of cyclin EL protein caused by Adwt and Adhz63 infection (Fig. 2.6C, lanes 4 and 6). To sum up, these data show that inhibition of CDK2 with Ros repressed pRb phosphorylation and inhibited viral replication. 
A

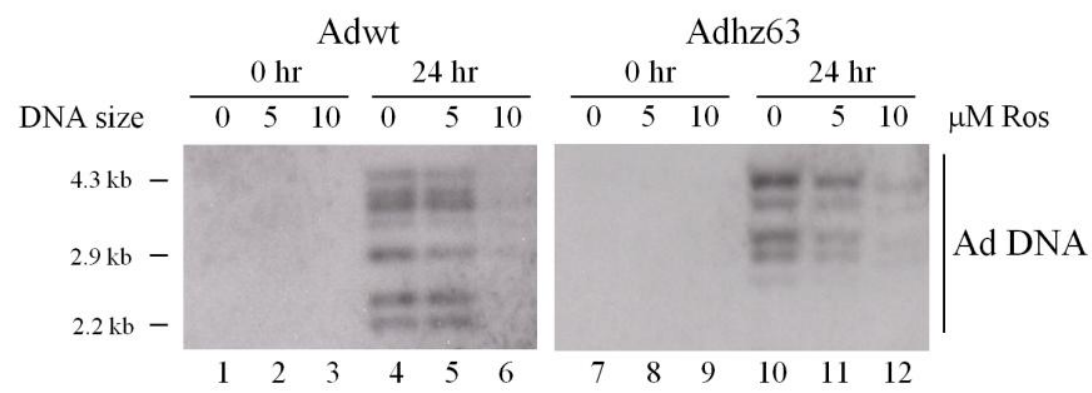

B

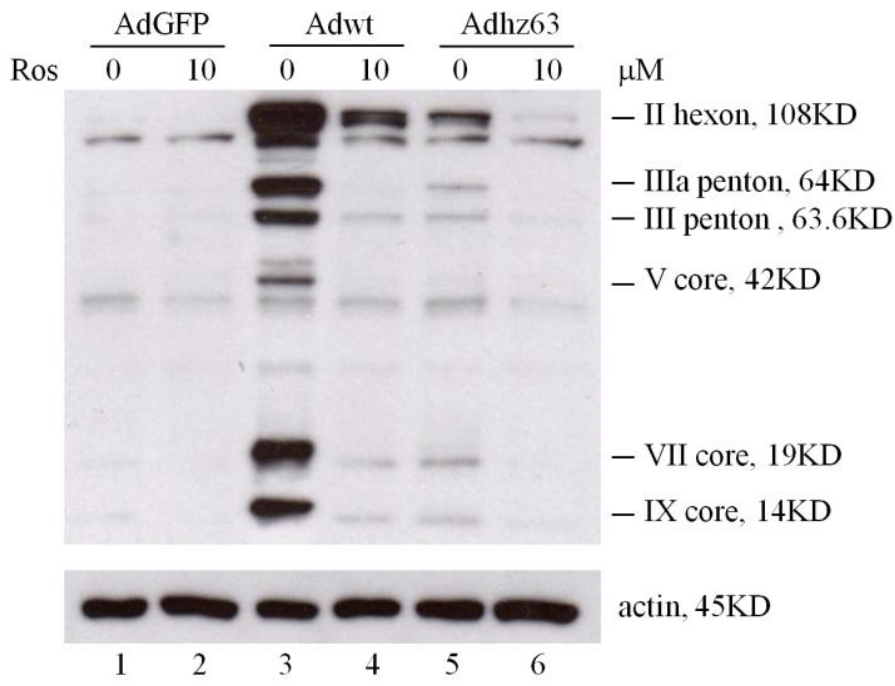

C
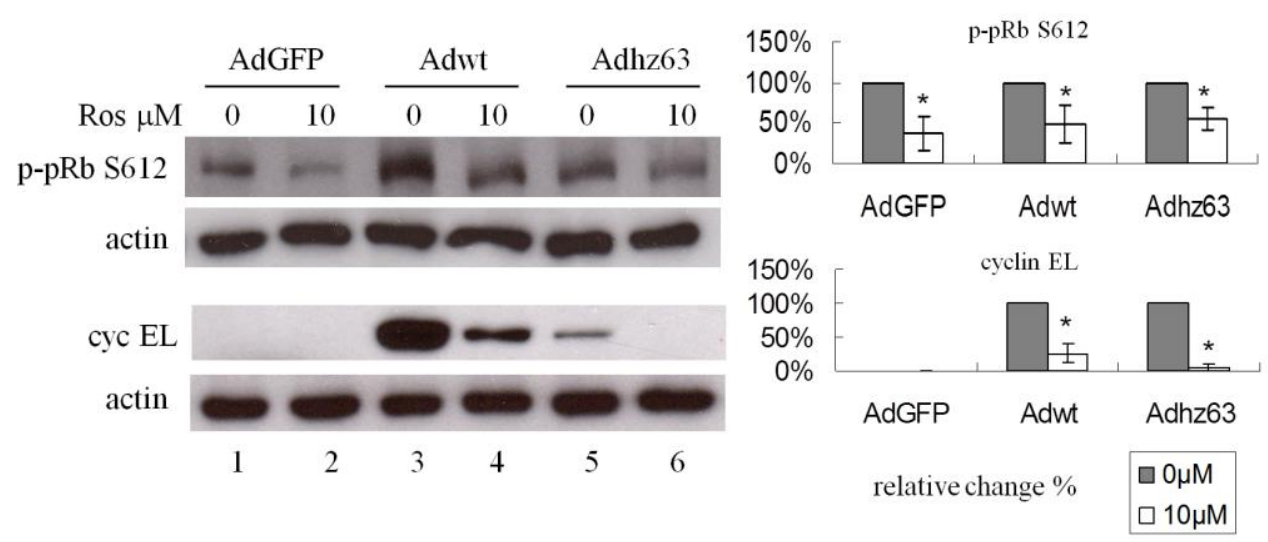
Figure. 2.6. Effects of Ros on viral DNA synthesis, viral capsid proteins, virus-induced cyclin E and phospho-pRb S612. (A) A549 cells were collected at $0 \mathrm{hr}$ and $24 \mathrm{hr}$ p.i. Viral DNA synthesis was determined by Southern blot. At 24 hr p.i., cells were harvested and cell lysates were immunoblotted for (B) adenovirus type 5 capsid proteins, (C) cyclin EL, p-pRb S612, and actin. Actin was used as a loading control. * $\mathrm{P}<0.05$ compared with the $0 \mu \mathrm{M}$ roscovitine-treated group, Student's $t$-test. 
siRNA inhibiting CDK2 represses adenoviral replication by preventing pRb phosphorylation. Since the chemical inhibitor Ros may also influence other CDK and kinases (138), we also applied RNA interference to specifically silence CDK2 expression. We tested three different pairs of siRNA duplexes targeting CDK2 on the coding region and showed that all CDK2 siRNAs dramatically inhibited CDK2 expression without the detectable influence on the non-targeted CDK4 in A549 cells (Fig. 2.7A). To evaluate the effects of CDK2 on the cellular protein production in response to viral infection, A549 cells were infected with Adwt or Adhz63 after treatment with CDK2 siRNA duplex or a non-specific control siRNA for 48 hours. Figure 2.7B, representing one of the three repeated experiments, shows that blockage of CDK2 expression with siRNA partially inhibited Adwt and Adhz63-induced CPE. Inhibition of CDK2 expression with siRNA resulted in the decreases of Adwt titer from $1.4 \times 10^{9}$ (control siRNA) to $2.8 \times 10^{8}$ (specific siRNA) and Adhz63 titer from $1.2 \times 10^{8}$ to $4.2 \times 10^{7}$ (Fig. 2.7C). The effect of CDK2 siRNA on viral replication is statistically significant; the titers decreased about 5 fold for Adwt $(P=0.0005)$ and 3 fold for Adhz63 $(P=0.03)$.

We also evaluated the effects of CDK2 on the cellular protein production in response to viral infection. As we expected, the CDK2 siRNA specifically repressed the production of CDK2 protein and decreased pCDK2 T160 in Adwt and Adhz63-infected cells (Fig. 2.7D, lanes 2 and 4). Repression of CDK2 also resulted in reduced CDK2-specific phosphorylation on $\mathrm{pRb}$, but did not decrease $\mathrm{pRb}$ protein levels. In addition, we observed that treatment with CDK2 siRNA 
repressed specifically Ad-induced cyclin EL, but not cyclin E (Fig. 2.7D, lanes 2 and 4). This agreed our findings with Ros (Fig. 2.6C) showing that specifically inhibiting CDK2 with siRNA significantly repressed viral production, which correlated with the decreased CDK2 activation and phosphorylation on pRb in cancer cells. 
A

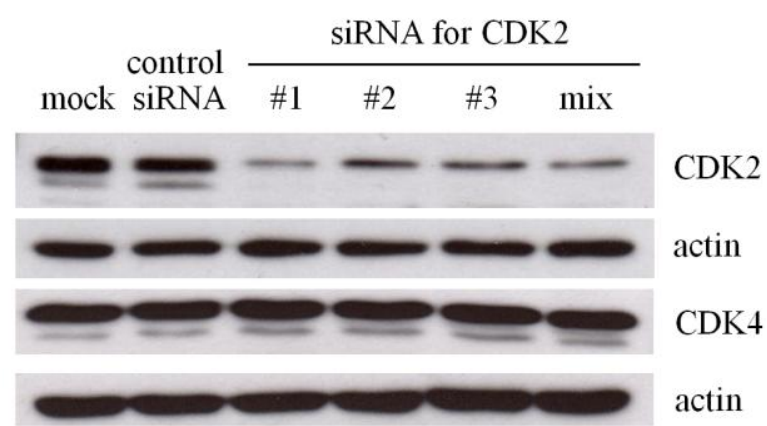

B

AdGFP

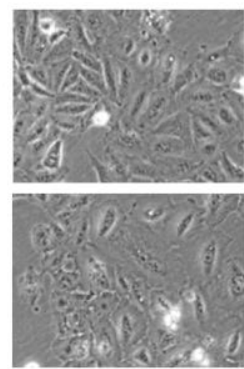

Adwt

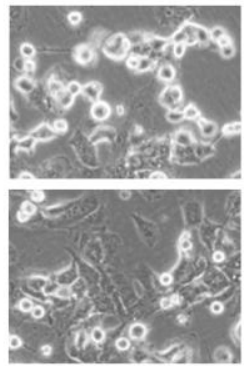

Adhz63

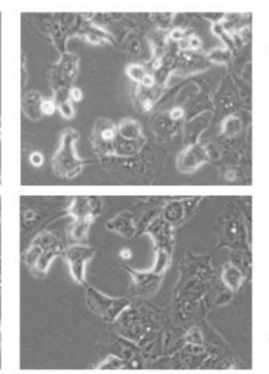

A549

C
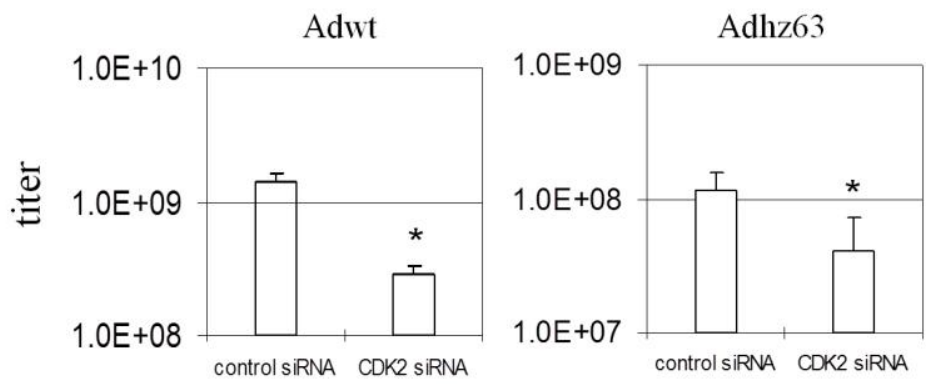

D

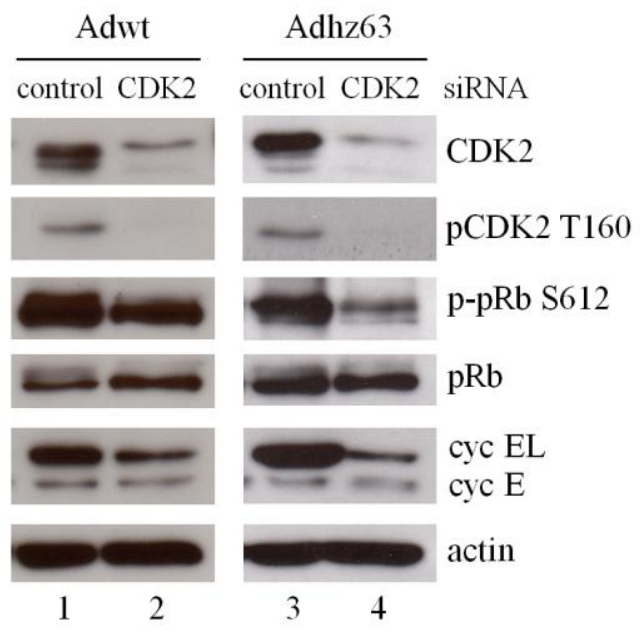


Figure 2.7. Effects of CDK2-specific siRNA on Ad replication in A549 human lung cancer cells. (A) A549 cells were transfected with 200 nM siRNA duplexes targeting different coding regions of CDK2. "Mix" represents the mixture of three pairs of siRNA duplexes (\#1, \#2 and \#3). Cells were harvested at $48 \mathrm{hr}$ after transfection. Cell lysates were immunoblotted for CDK2, CDK4, and actin. (B) At $48 \mathrm{hr}$ after transfection with CDK2 siRNA duplex or a duplex of non-specific control siRNA, cells were infected with Adwt or Adhz63 at an MOI of 5. CPE was photographed at $48 \mathrm{hr}$ p.i. All microscopy is originally at a magnification of $x 100$. (C) The viral titers were determined at $48 \mathrm{hr}$ p.i. with the infection unit method. The values are means \pm S.D. of independent triplicate. ${ }^{*} P<0.05$ compared with the control group, Student's $t$-test. (D) The infected cells were harvested at $24 \mathrm{hr}$ after infection. Cell lysates were immunoblotted for CDK2, pCDK2 T160, pRb, p-pRb S612, cyclin E, cyclin EL, and actin. Actin was used as a loading control. 


\section{siRNA inhibiting CDK2 repressed wild-type adenovirus replication in normal}

cells. Considering that the control of $\mathrm{G}_{1}$ exit is generally abnormal in cancer cells, we verified the role of CDK2 in Ad replication in WI-38 human diploid cell line that was derived from the normal embryonic lung tissue (139). As Adhz63 poorly induces cyclin EL and replicates in WI-38 cells (Fig. 2.1), we therefore investigated Adwt replication in WI-38 cells with the non-replication AdGFP as a negative control. WI-38 cells were infected with AdGFP or Adwt after transfected with CDK2 siRNA duplex or a non-specific control siRNA for $48 \mathrm{hr}$. Figure 2.8A, representing one of the three repeated experiments at $72 \mathrm{hr}$ p.i., shows that blockage of CDK2 expression with siRNA partially inhibited Adwt-induced CPE as we observed with Adwt-infected A549 cells. Inhibition of CDK2 expression with the specific siRNA caused a significant decrease of Adwt titer from $2.2 \times 10^{8}$ to $1.8 \times 10^{7}(P=0.03$, Fig. 2.8B). In addition, CDK2 repression also dramatically inhibited the viral DNA synthesis (Fig. 2.8C, lane 4) and capsid protein production (Fig. 2.8D, lane 4).

The level of cellular proteins in response to viral infection altered by CDK2 inhibition was also examined in WI-38 cells. For cells treated with the non-specific control siRNA, we were unable to detect pCDK2 T160 and cyclin E in WI-38 cells treated with AdGFP (Fig. 2.8E); this is related to the strict control of cyclin E expression in WI-38 cells. Adwt infection significantly increased CDK2, pCDK2 T160, and cyclin EL (Fig. 2.8E, comparing lanes 1 and 3). Inhibition of CDK2 by the siRNA repressed the pCDK2 T160, phospho-pRb S612, and cyclin EL 
induced by Adwt infection (Fig. 2.8E, comparing lane 1 with 2 and lane 3 with 4). Adwt infection and siRNA treatment did not show significant effects on pRb (Fig. 2.8E). Taken together, the results suggest that CDK2 activated by Ad-induced cyclin EL plays a general and important role in the adenoviral replication in normal cells. 
A

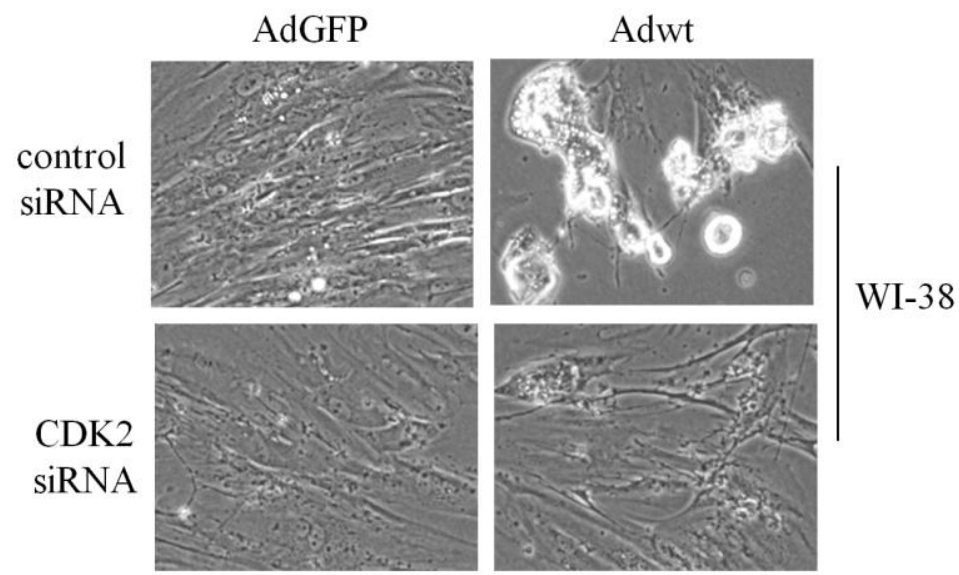

B

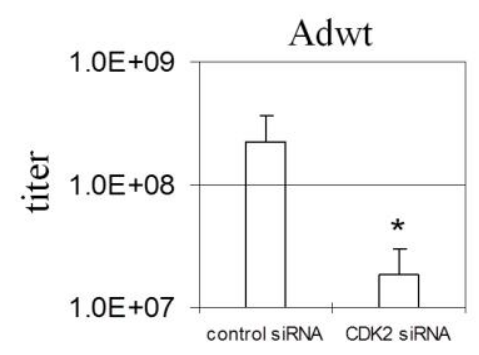

D

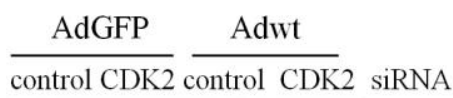
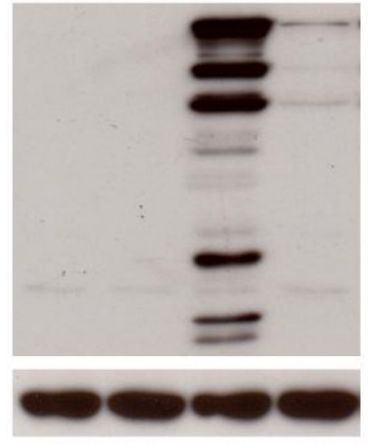

$\begin{array}{llll}1 & 2 & 3 & 4\end{array}$
C

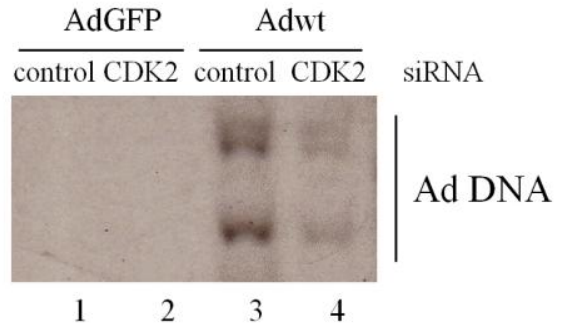

E

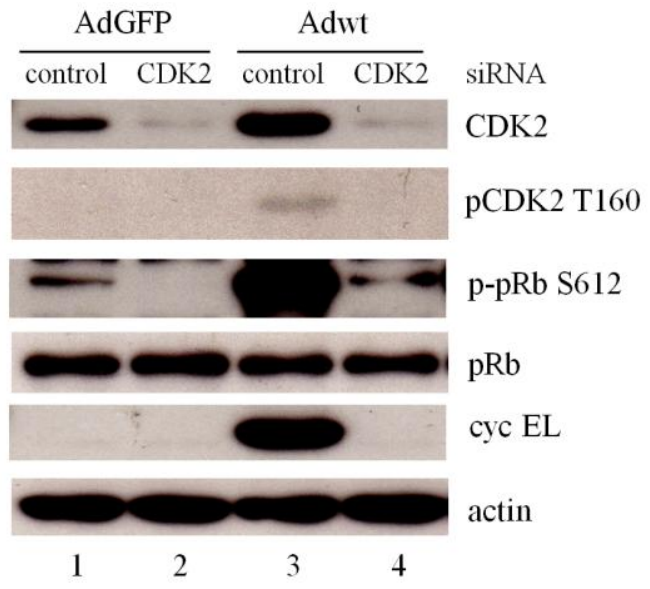


Figure 2.8. Effects of CDK2-specific siRNA on Ad replication in WI-38 human lung fibroblast cells. WI-38 cells were transfected with CDK2 siRNA duplex or a duplex of non-specific control siRNA. At $48 \mathrm{hr}$ after transfection cells were infected with AdGFP, Adwt, or Adhz63 at an MOI of 5. (A) CPE was photographed at $72 \mathrm{hr}$ p.i. All microscopy was originally at a magnification of $x 100$. (B) The viral titers were determined at $72 \mathrm{hr}$ p.i. with the infection unit method. The values are means \pm S.D. of independent triplicate. ${ }^{*} \mathrm{P}<0.05$ compared with the control group, Student's $t$-test. (C) Cells were collected at $48 \mathrm{hr}$ p.i. and viral DNA synthesis was determined by Southern blot. Cell lysates were immunoblotted (D) for adenovirus type 5 capsid proteins and for (E) CDK2, pCDK2 T160, pRb, p-pRb S612 and cyclin EL. Actin was used as a loading control. 


\subsection{Discussion}

By using multiple cell lines (A549, WI-38, HCT116, RKO, HepG2, Hep3B, Saos2, HeLa, MDA-MB-231, and HT29), we previously have shown that induction of cyclin EL is required for Ad replication and correlated with oncolytic selectivity of E1B55K-deleted Ad $(56,79)$. In this report, we extended the study and focused on cyclin E and CDK2 interaction in human lung cells that are natural host cells for human adenoviruses; we demonstrated that CDK2 activation by cyclin EL is a critical molecular step in Ad replication. Three lines of evidence support the importance of activation of CDK2 by cyclin EL in Ad replication. First, Ad-induced cyclin EL directly interacted with CDK2 and formed cyclin EL/CDK2 complex, leading to specifically increased phosphorylation of CDK2 and pRb (CDK2 at T160 and pRb at S612). Second, the CDK2 chemical inhibitor roscovitine decreased viral replication. Finally, the siRNA specifically inhibiting CDK2 repressed the viral replication with the decrease in pRb phosphorylation. These

three lines of evidence support the hypothesis that Ad-induced cyclin EL activates CDK2, which targets the transcriptional suppressor $\mathrm{pRb}$, controlling cellular and viral gene expression for productive viral replication (Fig. 2.9). 


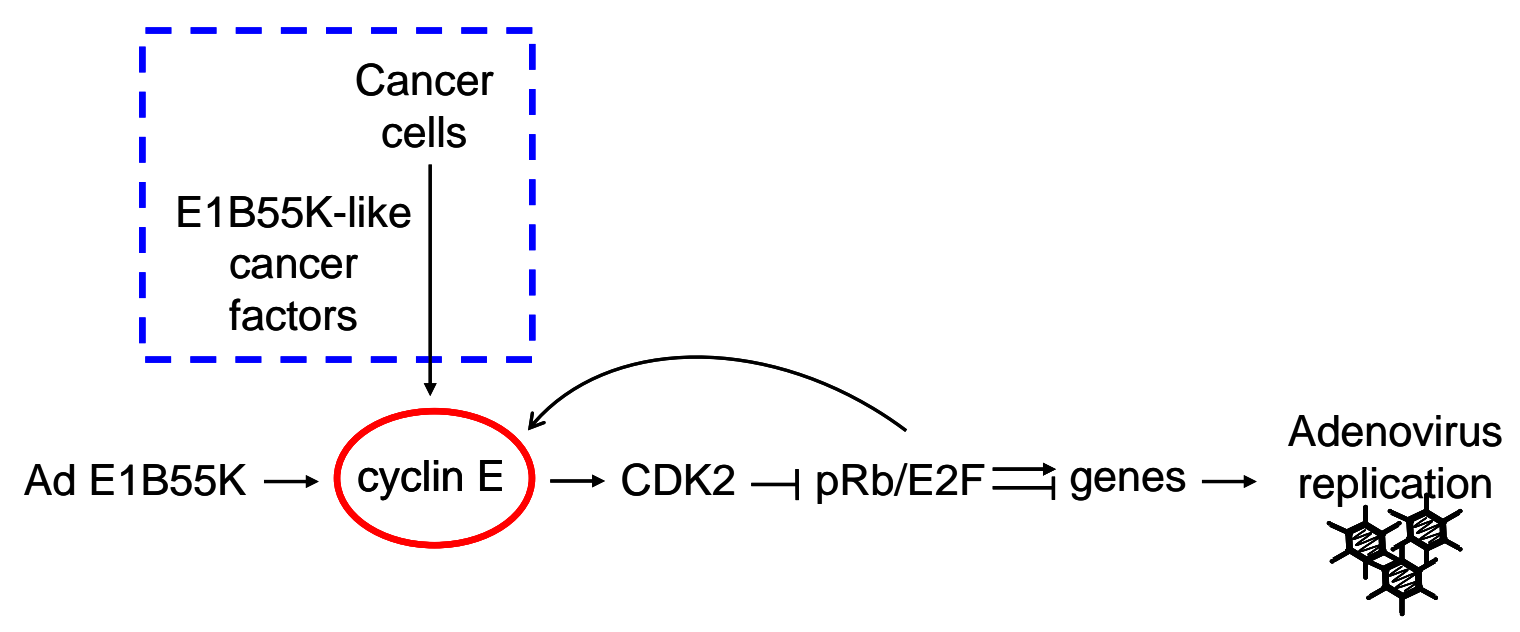

Figure 2.9. Proposed mechanism of cyclin E function in Ad replication. In Ad-infected cells, Ad E1B55K has a function to enhance cyclin E expression. This E1B55K function is not required for virus replication in cancer cells, which may provide E1B55K-like factors to relax cyclin E regulation and promote cell cycle progression. Cyclin E binds to and activates CDK2. Subsequently, the active pCDK2 phosphorylates the transcriptional repressor $\mathrm{pRb}$, leading to the expression of multiple genes, including cyclin E, to provide a suitable cellular environment for viral replication. 
Cyclin E and the large form cyclin EL are generated from alternative splicing. The translation of cyclin EL is initiated at an ATG codon located in exon 2 and cyclin E is from the ATG codon in exon $3(80)$. It has been reported that cyclin EL is found predominantly in breast tumor cells with the abundant lower-molecular-weight (LMW) isoforms $(140,141)$. We previously constructed a plasmid, pTet-cycE, containing cyclin E cDNA that produces these two forms of cyclin E proteins (56). With this approach we clarified that the A549 cell line constitutively expresses the regular cyclin E protein (cyclin E), and Ad infection mainly induces the expression of large form cyclin E protein (cyclin EL) (56). It is still unclear why Ad infection mainly induces cyclin EL. Considering that cyclin E has CDK2-dependent (82) and independent functions that are related to participation in DNA replication licensing (83) and oncogenic transformation (84), we studied whether Ad-induced cyclin E may target and activate CDK2 in virus-infected cells for productive Ad replication.

We first examined the physical interaction between virus-induced cyclin EL and CDK2. The results indicated that Ad-induced cyclin EL preferentially associates with CDK2 protein (Fig. 2.2). We cannot exclude that endogenous cyclin E in A549 cells may also interact with CDK2; however, we observed that the increased CDK2 in the cyclin E/CDK2 immunocomplexes was associated with cyclin EL induction after infection with replication-competent Adwt and Adhz63. The results suggested cyclin EL highly interacts with CDK2 in Ad-infected cancer cells. In proliferating cells, the abundance of the cyclin E protein directly links to the 
formation of active cyclin E/CDK2 complex $(80,142)$. With an intact cyclin box and the C-terminal 50 amino acids, the full-length cyclin EL is able to bind and activate CDK2 as cyclin E $(82,84)$. Consistent with our finding, previous studies showed that the addition of exogenous cyclin EL increases the formation of cyclin EL/CDK2 complex correlating to the increased activity and phosphorylation of CDK2 in human lung fibroblasts (80) and breast cancer cells (143). We reason that Ad-induced cyclin EL may have a strong affinity to CDK2 in the cellular environment affected by Ad infection.

We identified that Ad-induced cyclin EL correlates with the increase in phosphorylation of CDK2 at T160 and pRb at S612 (Figs. 2.3B and 2.4A). Three phosphorylation sites have been identified in CDK2 (125). T160 phosphorylation is essential for CDK2 activity, while T14 and Y15 phosphorylation cause an inhibitory effect. The retinoblastoma tumor suppressor $\mathrm{pRb}$ is inactivated by CDK's phosphorylation and enables E2F transcription factor to be released from the $\mathrm{pRb} / \mathrm{E} 2 \mathrm{~F}$ complex to carry out the downstream gene regulation $(144,145)$. Phosphopeptide analysis of pRb showed that $\mathrm{S} 612$ is one of the CDK2-preferred phosphorylation sites (130). We also examined the level of $\mathrm{pRb}$ with phosphorylation of T821 (CDK2-preferred) and S795 (CDK4-preferred); we did not detect any significant change at either of these two sites (Fig. 2.4A). Inhibition of CDK2 expression with the CDK2 siRNA repressed phosphorylation on CDK2 and pRb (Fig. 2.7D) and decreased viral replication (Fig. 2.7C). These results indicate that Ad-induced cyclin EL activates CDK2 by phosphorylating at T160, 
which then specifically introduces $\mathrm{pRb}$ phosphorylation at the $\mathrm{S} 612$ site.

The $p R b$ phosphorylation by cyclin EL/CDK2 may lead to regulation of multiple cellular and viral genes for productive Ad replication. Interestingly, Ad-induced cyclin EL expression was also inhibited by a CDK2 chemical inhibitor and CDK2 siRNA (Figs. 2.6C, 2.7D and 2.8E). It seems that inhibition of CDK2 interferes in the cyclin E induction via a loopback regulation (Fig. 2.9). Previous studies have reported that cyclin E gene is the downstream target of E2F $(146,147)$. In our previous work, we showed that the cyclin E promoter is more active in cancer cells and the promoter activity is further enhanced after Ad infection (56). We suggest that cyclin EL activates the cyclin EL-CDK2-pRb/E2F pathway and cyclin EL itself is also one of the targets of the pathway.

We detected a notable decrease of CDK inhibitors p21 and p27 in the Ad-infected cells. p21 and p27 inhibit the activity of cyclin/CDK complexes to prevent the cell-cycle progression, and their protein stability is also regulated by cyclin/CDK complexes $(134,148,149)$. Phosphorylation of $p 27$ by cyclin E/CDK2 causes $p 27$ degradation (148, 150). Montagnoli et al. (1999) showed that cyclin E/CDK2-dependent phosphorylation of p27 at threonine 187 facilitates the formation of a trimeric complex with cyclin E/CDK2 and leads to p27 ubiquitination (151). In agreement with our findings, recent studies also suggest that CDK may promote p21 degradation $(149,152)$. Thus, the activated cyclin E and CDK2 may decrease the CDK inhibitors p21 and p27 to benefit viral replication. 
In summary, our results showed that Ad-induced cyclin EL binds to and activates CDK2 that subsequently phosphorylates the transcriptional suppressor $p R b$, which can regulate expression of multiple cellular and viral genes, including cyclin E (Fig. 2.9). Our previous studies have shown that Ad E1B55K has a function to enhance cyclin E induction. In cancer cells, this E1B55K function is not critically required for cyclin $E$ induction and viral replication, likely because of deregulated cyclin E expression or having E1B55K-like cancer cellular factors. This study demonstrated that Ad-induced cyclin EL plays a critical role in Ad replication through activation of CDK2 that generates a suitable environment for viral replication. Our study reveals a new molecular basis for Ad replication in cancer cells that will guard us to develop new oncolytic vectors and therapeutic strategies. 


\section{CHAPTER III}

\section{ONCOLYTIC ADENOVIRUS TARGETS CYCLIN E OVEREXPRESSION AND INHIBITS TUMOR DEVELOPMENT IN VIVO}

\subsection{Introduction}

Oncolytic virotherapy with self-replicating and cancer-selective viruses has emerged as a new hope for cancer treatment. The therapeutic effects of oncolytic viruses are initiated from small amount of viruses and spread to surrounding tumor cells, and thus has been considered as an attractive drug platform $(4,153)$. Gene-attenuated replication-competent adenovirus (Ad) ONYX-015/dl1520 has been applied to several preclinical $(16,36,154)$ and clinical studies in the United States $(17,18)$. With the structure similar to dl1520 and slight modification, H101 has been commercially approved for cancer treatment in China.

Although dl1520 has achieved some measure of success in solid tumor treatment, the native E1a promoter on d1520 is constitutively active and thus leads to the safety concerns that virus may replicate in normal cells to cause toxicity (155-157). The major strategy to increase tumor specific replication of oncolytic Ads depends on transcriptionally regulating the essential E1A expression by tumor-specific promoters $(6,158)$. The proteins encoded by the E1a region are expressed immediately after infection, and these gene products modulate the cell cycle, 
recruit cellular proteins, and produce viral proteins to process viral DNA replication (37). By replacing native E1a promoter with cellular promoter preferentially active in tumor rather than normal tissues, oncolytic Ads can carry out more selective replication restricted in tumors. Some tumor-specific promoter based oncolytic Ads such as human telomerase reverse transcriptase (hTERT)-promoter driven OBP-301 (Telomelysin) (32) and prostate-specific antigen (PSA)-promoter driven CV706 (29) have been evaluated in clinical trials. Nevertheless, most known tumor-specific promoters are only active in a narrow range of tumors with relative weak activity compared with the constitutive viral promoters which are ubiquitously active (159-163). Also, our published microarray study indicates virus infection causes strong repression of various cellular promoters (79). Lacking consideration for the negative effects imposed by virus infection on those promoters may narrow our insight and thus hinder the future vector design.

Cyclin $E$ is known to regulate cell cycle progression (80), DNA replication (110, $111)$, and centrosome duplication $(112,113)$. Numerous types of cancers such as lung cancer and breast cancer are highly associated with dysregulation of cyclin E (87). Constitutive overexpression of cyclin E induces chromosome instability (89, 90), impairs normal cell cycle progression and triggers tumorigenesis in transgenic animal models $(86,114,115)$. Previously we have demonstrated that cyclin E overexpression is a molecular basis of selective oncolytic replication of E1b55K-deleted Ads in human cancer cells $(56,79)$. Ads induce cyclin E which activates CDK2 that targets the transcriptional repressor $\mathrm{pRb}$, turning on the 
downstream gene expression to adjust the cellular environment for productive viral replication (164). We also reported that cyclin E promoter, highly active in cancer cells, is further augmented during viral reproduction process $(56,165)$. The unique properties of cyclin E promoter in virus replication indicate its potential on the oncolytic vector design. Based on these findings, we generated a novel E1b-deleted Ad-cycE in which the E1a gene is under the control of the human cyclin E promoter. E1A expression driven by the cyclin E promoter in Ad-cycE can be enhanced by dual pathways. Since the cyclin E promoter is already highly active in many types of cancer cells, the initial E1A expression will be driven by activation of cyclin E promoter by cancer cellular factors, leading to selective viral replication. The activity of cyclin E promoter can also be further augmented by Ad itself as we have shown that cyclin E expression is significantly upregulated in cancer cells after Ad infection (Fig. 3.1). 


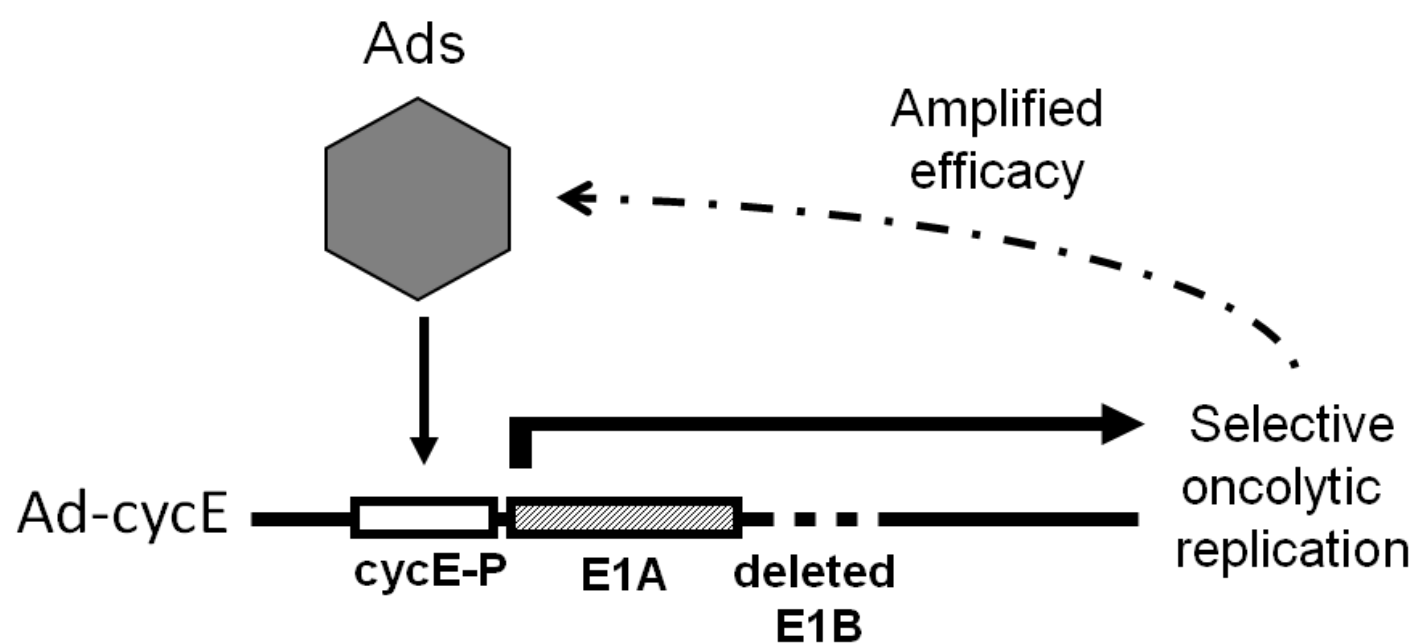

Figure 3.1. Proposed rationale of activating E1A transcription in Ad-cycE vector. Ad-cycE is an E1b-deleted oncolytic Ad carrying a cyclin E promoter (cycE-P) to drive an intact E1A expression cassette. With dual properties to target the cyclin E overexpression in cancer cells and virus-induced environment, cyclin E promoter may allow either the cellular factors from cancer cells or the viral factors from viral reproduction process to activate virus E1A transcription in Ad-cycE. 
Our results revealed that a novel therapy Ad-cycE targets cyclin E overexpression in cancer cells, significantly represses tumor growth and prolonged survival of nude mice bearing human lung cancer xenograft. The vector also shows selective and efficient antitumor effects on cancer cell lines reported as non-permissive for dl1520 replication. This study not only provided the attractive insight for the future design of the oncolytic Ad vectors, but also unraveled the potent antitumor efficacy by targeting cyclin E overexpression in cancer cells and viral reproduction process. 


\subsection{Materials and Methods}

Cell lines and culture conditions. Human embryonic kidney HEK 293 (ATCC no. CRL-1573), lung fibroblast WI-38 (ATCC no. CCL-75), lung cancer A549 (ATCC no. CCL-185) and H1299 (ATCC no. CRL-5803), prostate cancer DU-145 (ATCC no. HTB-81) and LNCaP (ATCC no. CRL-1740), osteosarcoma Saos2 (ATCC no. HTB-85), and breast cancer MDA-MB-231 (ATCC no. HTB-26) cell lines were purchased from the American Type Culture Collection (Rockville, MD). HEK 293, A549 and MDA-MB-231 cells were cultured in DMEM. Saos 2 cells were cultured in McCoy's 5A. LNCaP and H1299 cells were cultured in RPMI-1640 medium. WI-38 cells were cultured in minimal essential medium (MEM) Alpha GlutaMAX with $0.1 \mathrm{mM}$ non-essential amino acids and $1.0 \mathrm{mM}$ sodium pyruvate. All media were supplemented with $10 \%$ fetal bovine serum (FBS) and penicillin/streptomycin $(100 \mathrm{U} / \mathrm{ml})$. Cells were cultured in a $5 \% \mathrm{CO}_{2}$ incubator at $37^{\circ} \mathrm{C}$. All cell culture reagents were obtained from Gibco BRL (Bethesda, MD) and Corning Cellgro (Manassas, VA).

Adenoviral vectors. Wild-type adenovirus type 5 (Adwt, ATCC no. VR-5) was used as a replication-competent control. AdCMV/GFP, an Ad vector with E1 deletion carrying a green fluorescent protein (GFP) constructed in our laboratory (166), was used as a replication-defective control. Ad dl1520 is a E1b55K mutant which contains an 827-bp deletion and a point mutation to generate a premature stop codon in the E1B55K coding region (15). Adhz55 is a E1b-deleted oncolytic vector carrying prostate-specific ARR2PB promoter (167) driving an intact E1A 
expression cassette constructed in our laboratory. Ad-cycE is a novel E1b-deleted oncolytic vector carrying a human cyclin $\mathrm{E}$ promoter driving an intact $\mathrm{E} 1 \mathrm{~A}$ expression cassette. To prepare Ad-cycE vector, 671 bp of human cyclin $E$ promoter (GenBank ID: X95406) $(168,169)$ was released by digesting pGL-2-cycE promoter 10-4 plasmid (Addgene, Cambridge, MA) with Smal and the fragment was ligated into the shuttle plasmid pMEa301 which contains promoterless E1a region and the deletion of $E 1 b$ to generate pBZ400 plasmid. The pBZ400 plasmid contains cyclin E promoter, together with entire E1a open reading frame and E1b deletion. pBHGE3 plasmid (122) containing Ad genomic DNA and pBZ400 plasmid were cotransfected into 293 cells. Ad-cycE was rescued from cells after transfection and contains a human cyclin E promoter to control E1a open reading frames (ORF) with the deletion of E1b region. All of the vectors created and used in this study are based on the backbone of wild-type Ad type 5 .

Cytotoxicity assay. Cells were seeded into 24-well plates at a density of $2.5 \times 10^{4}$ (cells/well) and cultured under the indicated conditions. Cytotoxicity was assessed with crystal violet staining (170). Cells were fixed with $3.7 \%$ paraformaldehyde and stained with $1 \%$ crystal violet followed by washing with water to remove excess dye. The dye was solubilized with $2 \%$ SDS and the absorbance of the solubilized stain was measured at $590 \mathrm{~nm}$ using a Synergy HT Multi-Mode Microplate Reader (Bio-Tek, Winooski, VT). The OD values were quantitated into the cell viability $\%$ by the formula, cell viability $\%=(O D$ value of 
experimental group / OD value of control group) $\times 100 \%$. The mock-control group was calculated as $100 \%$ of cell viability in the assay (171).

Western blot analysis. Infected cells were harvested at indicated time points and lysed with CDK2 lysis buffer $(20 \mathrm{mM}$ Tris $\mathrm{pH} 7.5,150 \mathrm{mM} \mathrm{NaCl}, 5 \mathrm{mM} \mathrm{MgCl} 2$, 0.5\% Nonidet P-40, 0.1\% Brij 35, $5 \mathrm{mM}$ sodium glycerophosphate, $1 \mathrm{mM}$ sodium vanadate, $1 \mathrm{mM}$ dithiothreitol). The Western blot analyses were performed as described previously (123). Briefly, $80 \mu \mathrm{g}$ of cell lysates were electrophoresed through $12 \%$ SDS-polyacrylamide gels and transferred onto an Immobilon-P Membrane (Millipore, Billerica, MA). The primary antibodies used in this study were rabbit anti-cyclin E (M-20), (Santa Cruz Biotechnology, Santa Cruz, CA), mouse anti-adenovirus type 5 E1A (BD Pharmingen, San Jose, CA). Actin was used as an internal control. The membranes were then incubated with anti-mouse immunoglobulin $G$ ( $\lg G$ ) or anti-rabbit IgG peroxidase-linked species-specific whole antibody (GE Healthcare, Piscataway, NJ). Chemiluminescent detection was performed with ECL reagents according to the supplier's recommendations (GE Healthcare).

Viral titration. Total infected cells and culture supernatants were collected at the indicated time points and lysed to release virus particles with three cycles of freezing and thawing. The viral titers were determined by the infective unit method as described previously $(120,121)$. Briefly, HEK 293 cells were seeded in 96-well plates at a density of $10^{3}$ (cells/well) and then infected with 10-fold serially diluted 
viruses. CPE was recorded and scored after incubation for 7 days.

Lung cancer xenograft study. Female athymic nude mice (NCr-nu/nu) were obtained from National Cancer Institute (Bethesda, MD). $5 \times 10^{6}$ A549 human lung cancer cells were subcutaneously injected into the flanks of mice (aged 6 weeks). Once palpable tumors were established (mean of tumor sizes approximates to $20-25 \mathrm{~mm}^{3}$ ), the mice were randomized and received $5 \times 10^{8} \mathrm{IFU}$ of AdGFP or Ad-cycE in $50 \mu \mathrm{L}$ PBS every 2 days for total 4 treatments. The tumors were measured every 3 days until the tumor volume was greater than $1000 \mathrm{~mm}^{3}$. The tumor volume was determined by externally measuring in 2 dimensions with a caliper and calculated based on the following equation: $V=(\mathrm{L}$ $\left.X W^{2}\right) / 2$, where $L$ is length and $W$ is width of the tumor. Animal experiments were performed according to the institutional guidelines approved by the University of Louisville Institutional Animal Care and Use Committee. Survival data were plotted based on Kaplan-Meier method, followed by the analysis of log-rank test with SPSS statistical software (version 19.0; SPSS, Inc., Chicago, IL) (172).

Immunohistochemistry. Tumors were harvested one week after the fourth treatment in vivo, embedded in optimal cutting temperature compound (O.C.T.) (Sakura Finetek, Torrance $\mathrm{Ca}$ ), and stored at $-20^{\circ} \mathrm{C}$. The tumor sections and immunohistochemical staining were performed as previously reported (173). Briefly, slides were air-dried, fixed in cold acetone at $4^{\circ} \mathrm{C}$ for $20 \mathrm{~min}$. The endogenous peroxidase activity was blocked by $3 \%$ hydrogen peroxide for 10 
mins and washed with PBS three times, followed by incubation with SuperBlock (37515, Pierce Biotechnology, Rockford, IL) at room temperature for $30 \mathrm{~min}$. All antibodies were applied in SuperBlock. Sections were incubated with goat-anti-adenovirus polyclonal antibody (AB1056, Millipore, Billerica, MA), diluted 1:800, for $1 \mathrm{hr}$ at room temperature. The antibody staining signals were amplified by a biotinylated anti-goat IgG diluted 1:200 in conjunction with VECTASTAIN avidin-biotin complex method kit (Vector Laboratories, Burlingame, CA). Visualization was achieved using 3,3-diaminobenzidine tetrahydrochloride (ImmPACT DAB peroxidase substrate; Vector Laboratories). Slides were counterstained with hematoxylin and photographed at X200 and X400 magnification and in an Olympus BX53 microscope (Olympus, Center Valley, PA).

Statistical analyses. All above experiments, except specifically indicated, were repeated at least three times. Quantitation results were reported as means \pm standard deviation (S.D.). Statistical difference of the combination experiment was assessed with a Student's $t$-test. Statistical significance of difference was set at $p$ $<0.05$. 


\subsection{Results}

Ad-cycE induces more significant oncolytic effects on prostate cancer cells than prostate cancer-specific oncolytic Adhz55. Ad-cycE is an E1b-deleted vector with the cyclin E promoter to replace the endogenous Ad E1a promoter. We compared Ad-cycE with Adhz55 which has the prostate cancer-specific ARR2PB promoter driving Ad E1a gene (167). Non-replicative AdGFP was used as a negative control, and wild-type Ad 5 (Adwt) was used as the positive virus replication control. We first examined the E1A expression at $24 \mathrm{hr}$ p.i. in LNCaP and DU-145 prostate cancer cells. Cells were infected with AdGFP, Adwt, Adhz55 or Ad-cycE at 5MOI. With the same infection condition, the E1A expression of Ad-cycE was higher than that of Adhz55 in LNCaP and DU-145 prostate cancer cell lines (Fig. 3.2A, comparing lanes 3 and 4,7 and 8). We then examined the cytotoxicity of Adhz55 and Ad-cycE on these two prostate cancer cell lines. Cells were infected with Adwt, Adhz55, Ad-cycE or AdGFP at 0, 0.5, 1, 2, 4, $8 \mathrm{MOI}$ for $96 \mathrm{hr}$ and the cytotoxicity were determined by crystal violet staining. Figure 3.2B showed that AdGFP did not induce significant cytotoxicity at different MOls. Both LNCaP and DU-145 prostate cell lines were sensitive to Adwt and Ad-cycE infection, but relatively resistant to Adhz55 (Fig. 3.2). 
A.

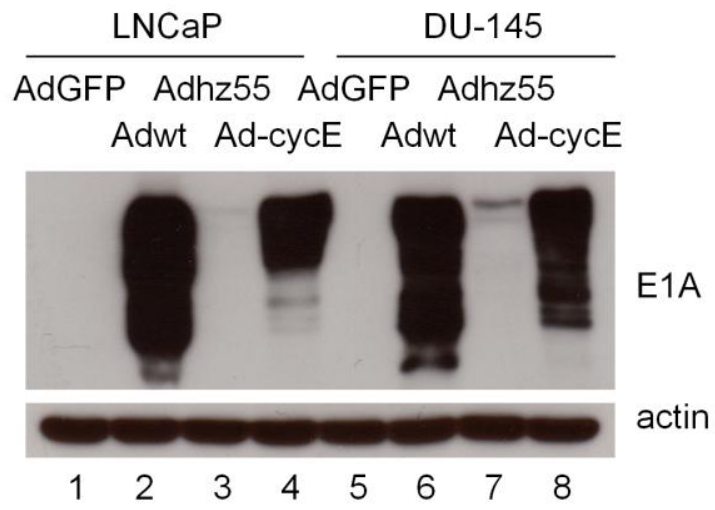

B.
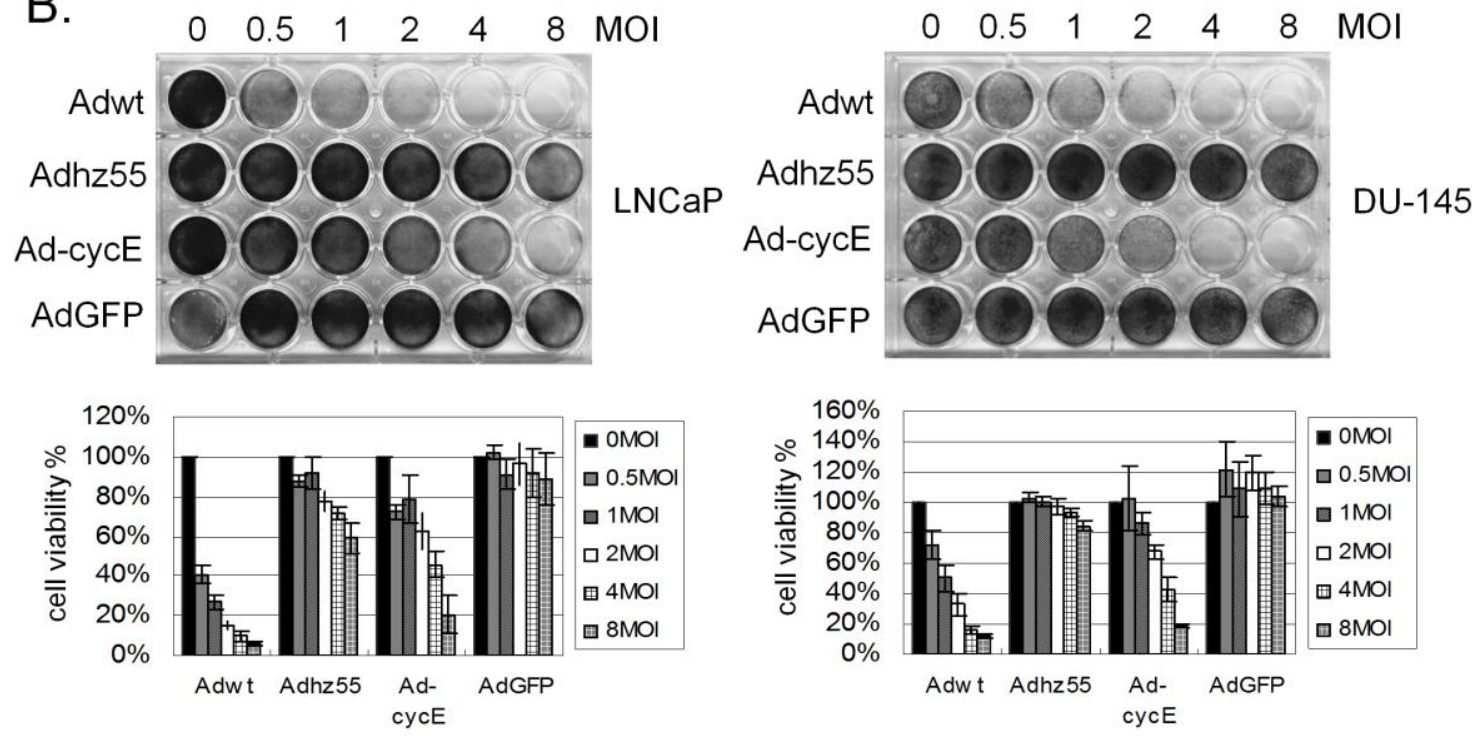
Figure 3.2. Comparison of prostate cancer-promoter driven Adhz55 and cyclin E-promoter driven Ad-cycE in prostate cancer cells. (A) LNCaP and DU-145 Cells were infected with AdGFP, Adwt, Adhz55 or Ad-cycE at $5 \mathrm{MOI}$ and collected at $24 \mathrm{hr}$ p.i., respectively. The cell lysates were immunoblotted for E1A and actin. Actin was used as a loading control. (B) Cells were seeded at a density of $2 \times 10^{4}$ and infected with Adwt, Adhz55, Ad-cycE or AdGFP at 0, 0.5, 1, 2, 4, and $8 \mathrm{MOI}$ for $72 \mathrm{hr}$. Cytotoxicity were determined with crystal violet staining and photographed. Data are representative examples of three independent sets of experiments and quantitated as cell viability \%. The values represent the means \pm S.D. of independent triplicate compared with the AdGFP-infected group of each dose. 


\section{Ad-cycE selectively destroys lung cancer cells and spares noncancerous}

lung cells. The oncolytic selectivity of Ad-cycE was determined with human A549 lung cancer cells and WI-38 noncancerous lung fibroblast. A549 cell line is a human lung cancer cell line with dysregulated cyclin E that can be significantly induced after Ad infection (56); WI-38 cell line has the properties of primary cells with a finite lifetime of 50 population doublings (174). As we mentioned above, AdGFP and Adwt were used as a negative and positive replication control, respectively. As E1b55K-deleted dl1520 with the native E1a promoter can replicate in different types of cancer cells, we particularly applied it as the oncolytic Ad control. Cytopathic effects (CPE), the classical characteristic of oncolytic virus replication, were recorded on Figure 3.3, showing morphological change as infected cells rounded up and detached from the cell monolayer. Without replication, AdGFP did not induce CPE on either WI-38 or A549 cells. Wild-type virus (Adwt) non-selectively induced CPE in both noncancerous WI-38 and A549 lung cancer cells; however Ad-cycE only induced significant CPE on A549 lung cancer cells but not on WI-38 cells as well as dl1520. In noncancerous WI-38 cells, Adwt significantly decreased cell viability to $30 \%(P=0.0000047$, compared with the AdGFP-infected group), while dl1520 and Ad-cycE do not cause significant changes $(P=0.28$ and 0.2 , compared with the AdGFP-infected group respectively). In A549 cells, Adwt decreased cell viability to 6\%, while dl1520 and Ad-cycE decreased cell viability to $40 \%$ and $25 \%$, respectively. It seems that Ad-cycE can destroy A549 lung cancer cells more efficiently than dl1520. 

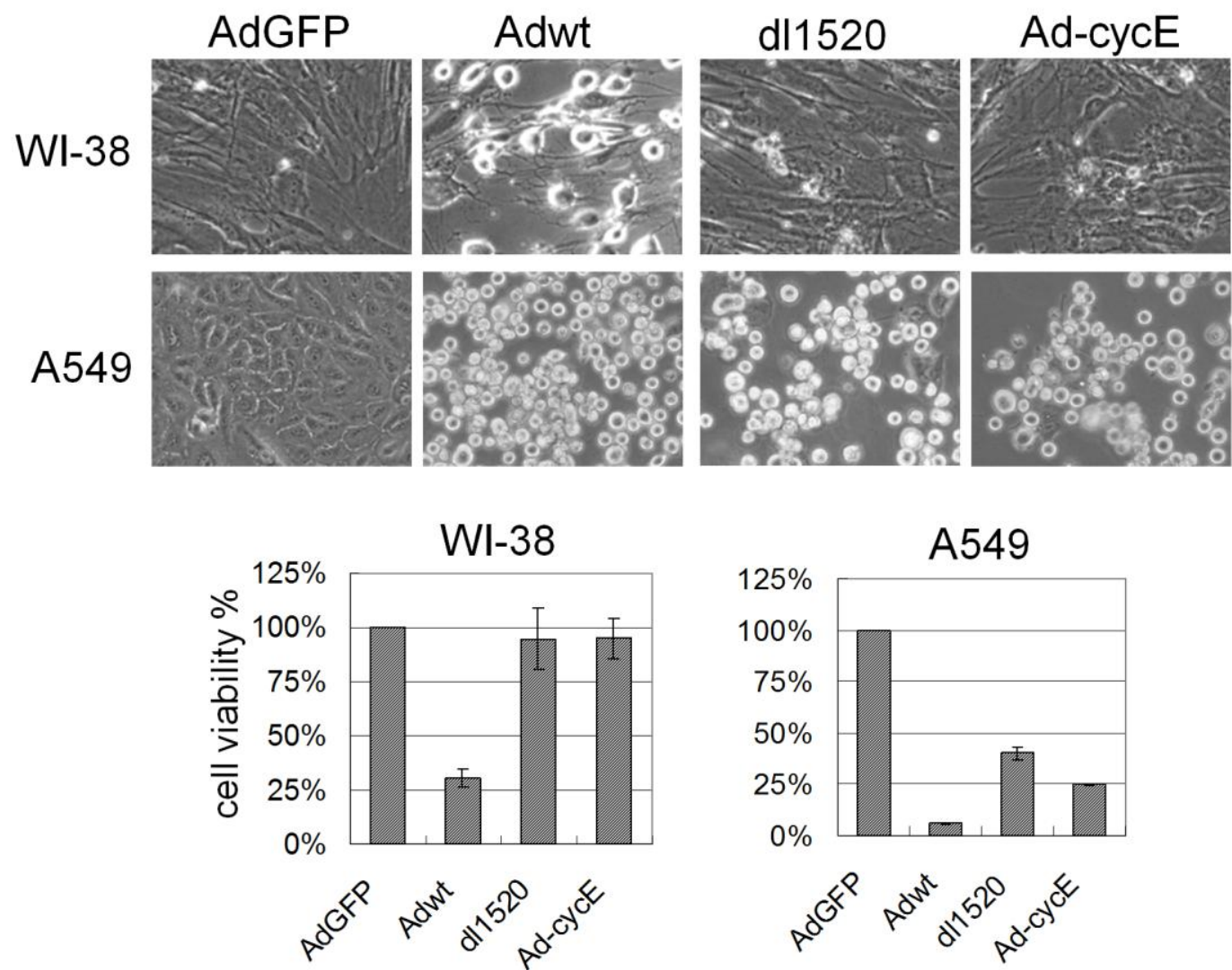

Figure 3.3. Cytopathic effects of human Ads on WI-38 and A549 cells. (A) Cells were infected with AdGFP, Adwt, dl1520 or Ad-cycE at 8 (for WI-38) or 5 MOI (for A549). CPE was observed at $72 \mathrm{hr}$ p.i and photographed with an inverted microscope Olympus CKX41. The cell viability \% was determined and the values represent the means \pm S.D. of independent triplicate compared with the AdGFP-infected group. 
Replication of Ad-cycE is activated in cancer cells. Ad E1A is the crucial protein which initiates the whole virus replication process and promotes cells to enter S phase (37). With E1a ORF under control of cyclin E promoter, we hypothesized that $\mathrm{E} 1 \mathrm{~A}$ transcription in $\mathrm{Ad}-\mathrm{cyc} \mathrm{E}$ is activated in cancer cells with cyclin E overexpression. A549 cells were infected with AdGFP, Adwt, dl1520 or Ad-cycE at $5 \mathrm{MOI}$ for $24 \mathrm{hr}$. As Figure 3.4A shown, no E1A expression was detected in non-replicative AdGFP with the deletion of $E 1$ region whereas the E1A expression of Ad-cycE was detected in A549 cells at a similar level to dl1520 and Adwt at $24 \mathrm{hr}$ postinfection (p.i.), suggesting E1A transcription of Ad-cycE is activated in A549 cells. To determine whether the activation of E1A transcription leads to Ad-cycE replication, virus production was monitored over time. The data showed that virus yield of Ad-cycE increased over time as well as Adwt and dl1520 (from $6.7 \times 10^{5}$ at $0 \mathrm{hr}$ to $8.3 \times 10^{8}$ at $72 \mathrm{hr}$ ) (Fig. 3.4B). Taken these data together, we showed Ad-cycE is a replication-competent virus in A549 lung cancer cells. 
A.

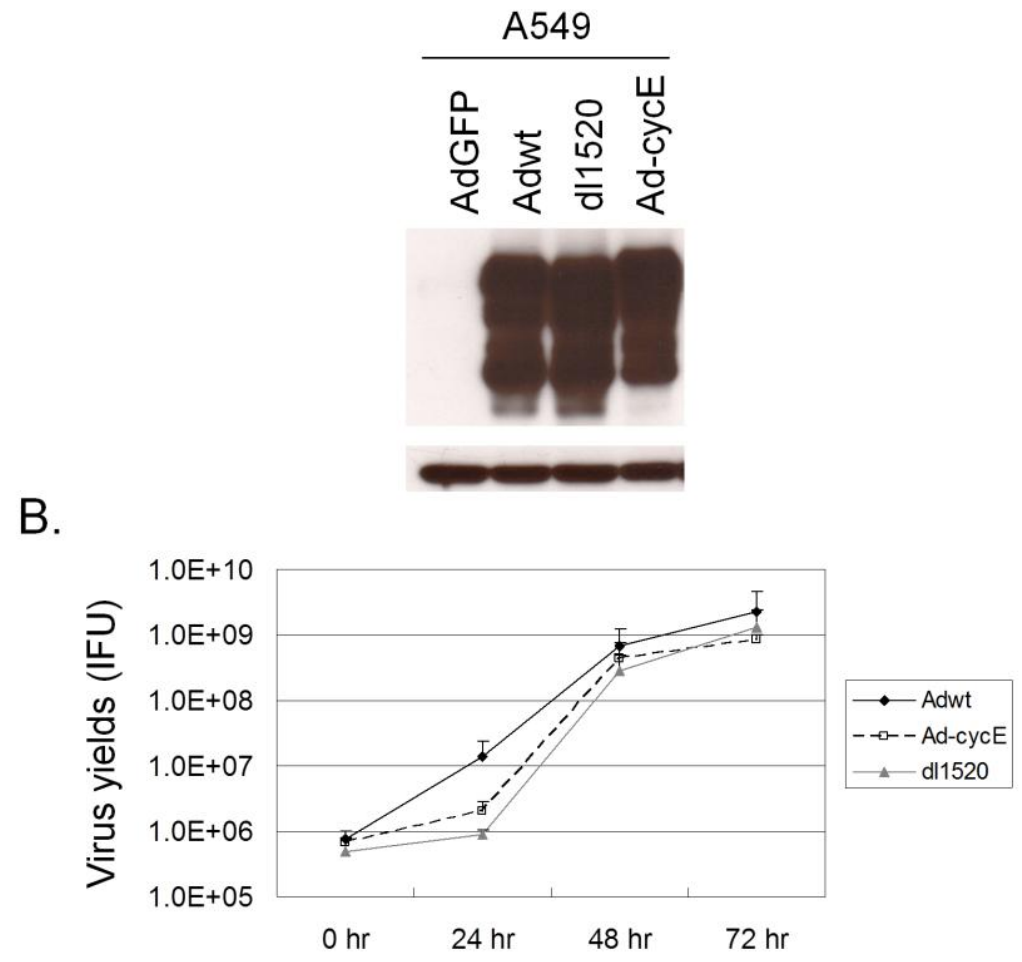

Figure 3.4. The expression of viral E1A and virus production in A549 cells. (A) Cells were infected with AdGFP, Adwt, dl1520 or Ad-cycE at $5 \mathrm{MOI}$ and collected at $24 \mathrm{hr}$ p.i. The cell lysates were immunoblotted for E1A protein and actin. Actin was used as a loading control. (B) The virus yields were determined at $0,24,48$, and $72 \mathrm{hr}$ p.i. with the infection unit method. The values represent the means \pm S.D. of independent triplicate. 
Oncolytic efficacy of Ad-cycE on multiple cancer cell lines. To detect the cytotoxicity of Ad-cycE, human A549 and H1299 lung cancer, MDA-MB-231 breast and Saos 2 osteosarcoma cancer cells were infected with Adwt, dl1520, Ad-cycE and AdGFP at the indicated MOI for $72-96 \mathrm{hr}$ and stained with crystal violet (Fig. 3.5). As a positive control, Adwt widely induced strong oncolysis in all cancer cell lines whereas a non-replicative control AdGFP did not cause oncolysis. A significant cytotoxicity was observed in all cancer cell lines infected with oncolytic dl1520 or Ad-cycE when compared to cells infected with non-replicative AdGFP. Cell viability was then quantified and each group was compared to the mock-infected group. In Saos 2 osteosarcoma and MDA-MB-231 breast cancer, Ad-cycE worked even better than dl1520 (Fig. 3.5, Boxed). This experiment showed that in addition to lung cancer cells, Ad-cycE can efficiently destroy a variety of cancer cells. 


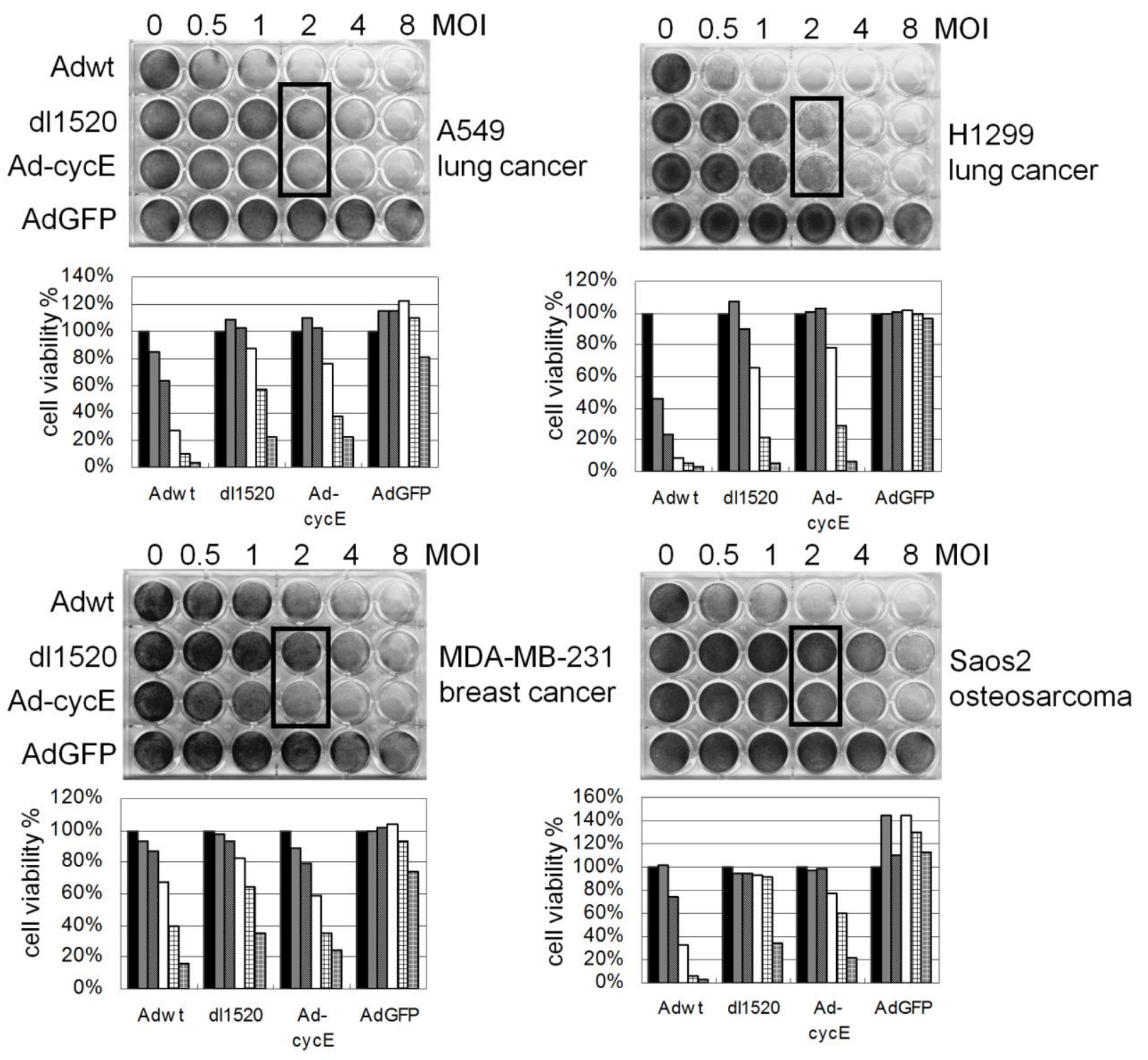


Figure. 3.5. Cytotoxicity of Ad-cycE in multiple cancer cell types. A549, H1299, Saos2, and MDA-MB-231 cells were seeded at a density of $2-5 \times 10^{4}$ and infected with Adwt, d11520, Ad-cycE or AdGFP at $0,0.5,1,2,4$, and $8 \mathrm{MOI}$ for 72-96 hr. Cytotoxicity were determined with crystal violet staining and photographed. Data were quantitated as cell viability \% values. The quantitated data of boxed areas indicated that with $2 \mathrm{MOI}$ of virus infection, dl1520 decreased the cell viability of $\mathrm{A} 549$ to $87 \%$ while Ad-cycE decreased it to $76 \%$; d 1520 decreased the cell viability of $\mathrm{H} 1299$ to $65 \%$ while Ad-cycE decreased it to $78 \%$; dl1520 decreased the cell viability of MDA-MB-231 to $83 \%$ while Ad-cycE decreased it to $59 \%$; dl1520 decreased the cell viability of Saos 2 to $92 \%$ while Ad-cycE decreased it to $77 \%$. 
Ad-cycE suppresses tumor growth in athymic nude mice. The therapeutic effects of Ad-cycE were further examined in the lung cancer subcutaneous xenograft model. Once palpable tumors were established, the mice were randomized and $5 \times 10^{8} \mathrm{IFU}$ of AdGFP or Ad-cycE in $50 \mu \mathrm{L}$ PBS was injected intratumorally every 2 days for total 4 treatments. The tumor sizes of mice were monitored over the time and the death of mice was recorded. The initial reduction of tumor volumes in Ad-cycE treated group was observed at day 9 after the first treatment. Mice treated with Ad-cycE exhibited significant suppression of tumor growth with $95 \%$ reduction in the mean tumor volume as compared with mice treated with control AdGFP at day 51 after the first treatment $(P=0.0008$, Fig. 3.6A). Once the tumor volume exceeded $1000 \mathrm{~mm}^{3}$, the mouse was sacrificed. The representative photographs of mice at the day of sacrifice revealed that in AdGFP-treated groups, tumor kept growing while Ad-cycE treatment suppressed tumor growth (Fig. 3.6B). In the Ad-cycE-treated group with total seven mice, two of them were tumor free after the treatment (no longer visible), three had decreased tumors all the time during the 130 days of follow-up, and two mice had recurring tumor growth at around 30 days after the first treatment (Fig. 3.6C). In an additional experiment, tumors from the mice that were injected with AdGFP or Ad-cycE were harvested 7 days after the last treatment and analyzed with immunohistochemical analysis to detect hexon protein expression. Hexon proteins, as the major coat protein of Ad capsids, represent the classic marker of virus particles (175). Hexon proteins were stained as the brown dots and localized within the cytoplasm (Fig. 3.6D, panel $\mathrm{c}$ and d). The results indicated a higher 
level of hexon expression in the tumor sections generated from A549 cell xenograft treated with Ad-cycE, suggesting the virus replicated within tumor tissues. The results reported here clearly demonstrate that Ad-cycE replicates in tumor tissues and strongly inhibits tumor growth, resulting in prolonged mice survival and potential antitumor efficacy. 
A.

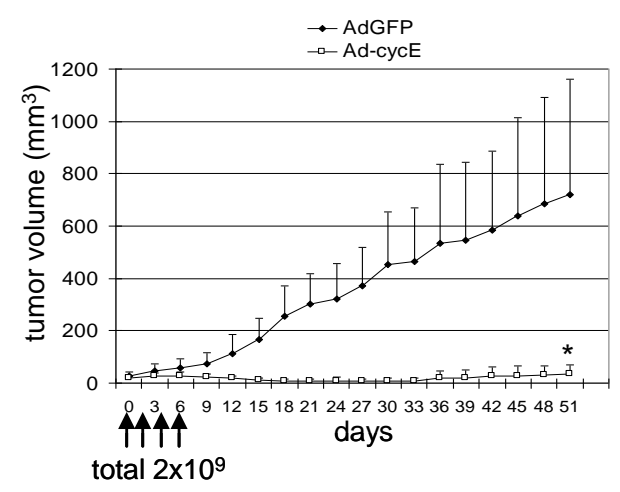

C.

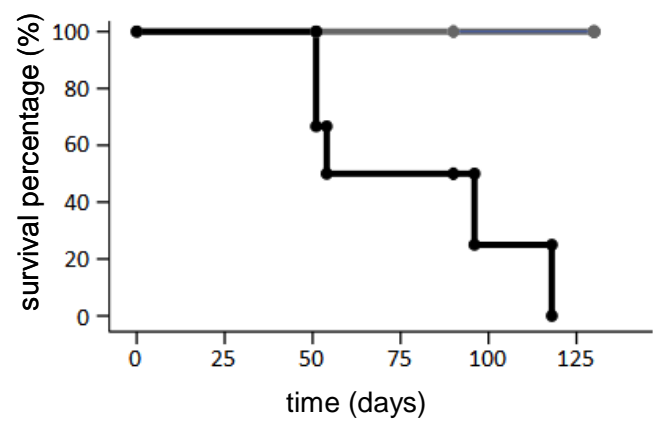

\begin{tabular}{|c|} 
Group \\
Ad-cycE \\
AdGFP \\
Ad-cycE \\
AdGFP
\end{tabular}
B.

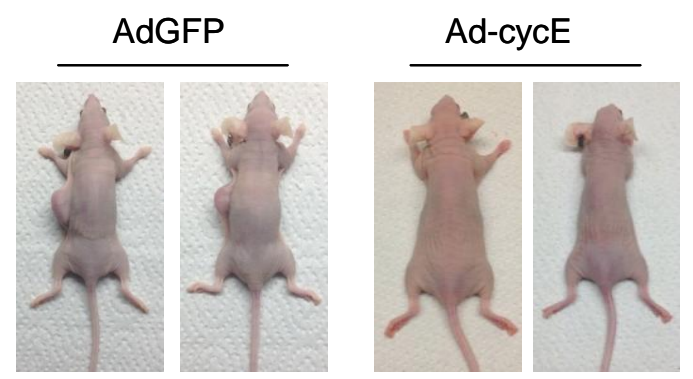

D.

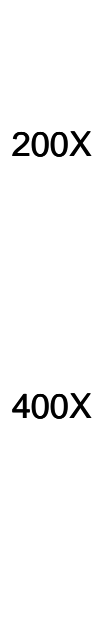

AdGFP

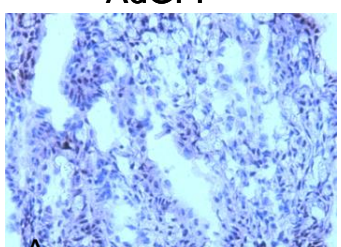

A.s and Com and

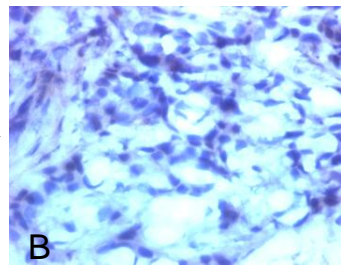

B.
Ad-cycE

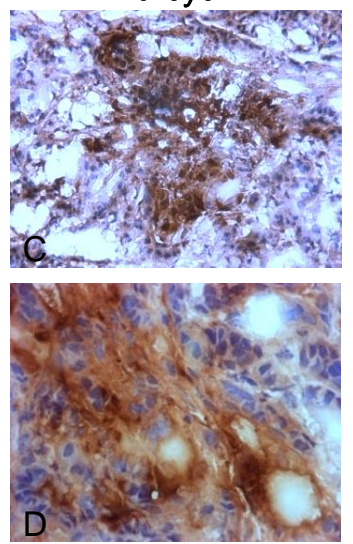

Hexon 
Figure 3.6. In vivo antitumor effects of Ad-cycE on subcutaneous A549 human lung cancer in nude mice. Mice carrying established A549 tumors were treated with control virus AdGFP ( $n=6)$ or Ad-cycE $(n=7)$ on days $0,2,4$, and 6 . The total viral dose was $2 \times 10^{9} \mathrm{IFU} /$ mouse. (A) The vertical arrow represents each treatment. Tumor volume (V) was plotted against time and was determined by the equation $V=\left(L \times W^{2}\right) / 2$, in which $L$ represents the length, and $W$ represents the width of the tumor. The values represent the means produce \pm S.D. * $\mathrm{P}<0.05$ compared with AdGFP control group, Student's $t$-test. (B) Representative mice from each group were photographed at the time of sacrifice. (C) Kaplan-Meier survival curves were plotted for both treatments (AdGFP $n=6$, Ad-cycE $n=7, P<0.05$, log-rank test). (D) Polyclonal antibody anti-adenoviral hexon was used to detect the production of adenoviral particles in tissue sections after treatment. The representative photographs were taken at original magnification X200 or X400 


\subsection{Discussion}

One of the significant features of cyclin $\mathrm{E}$ is its differential expression in cancer and normal cells. While dramatic overexpression of cyclin E has been detected in numerous cancers, the expression levels of cyclin $E$ are repressed in most nondividing normal cells (87). We previously have shown that cyclin E promoter is highly active in cancer cells and the promoter activity is further augmented during viral reproduction process $(56,165)$. The aim of this study was to evaluate a novel oncolytic Ad-cycE driven by the human cyclin E promoter with an entire E1b-deleted backbone. To date, none of known designs of tumor-specific oncolytic adenovectors took the virus-induced environment into consideration. This is the first oncolytic adenovector using a human cyclin E promoter combined with the strategy of entire E1b-deletion. As the replication of Ad-cycE depends on the activation of cyclin E promoter, the oncolysis of Ad-cycE is enhanced in cancer cells and repressed in normal cells. Our data showed that Ad-cycE selectively replicated in multiple cancer cells and inhibited the in vivo tumor growth in nude mice bearing lung cancers, leading to the prolonged survival time.

Most of the tumor-specific oncolytic adenoviruses are developed based on the promoters preferentially active in a specific type of cancers (158). Although regulating the viral gene expression by tumor-specific promoters is feasible, this approach can be hindered by the relatively weak activity (159-163) or virus-induced repression (79) on many tumor-specific promoters. Similar phenomenon was observed when we constructed prostate cancer-specific 
oncolytic Adhz55. We noticed that this kind of tumor-specific promoter somewhat may not drive E1A expression as well as constitutive viral promoters. Compared to prostate cancer specific promoter-based Adhz55, human cyclin E promoter-based Ad-cycE elicits better efficacy (Fig. 3.2). Also, the oncolytic efficacy of Ad-cycE was observed in different type of cancer cells (Fig. 3.5). Previously we have demonstrated that d11520 can induce cyclin E overexpression in the permissive cancer cells that support dl1520 replication but not in the restrictive cancer cells that are resistant for dl1520 replication (56). Here we noticed that the oncolysis induced by Ad-cycE is not only restricted in the reported permissive lung cancer cells A549 and H1299. In the reported restrictive cancer cells for dl1520 replication, Ad-cycE elicited better oncolytic efficacy than dl1520. While the exact mechanism(s) to induce oncolysis by Ad-cycE in the restrictive cancer cells for dl1520 replication remains to be investigated, it is possible that cyclin E promoter activated after Ad infection may benefit Ad-cycE replication in those restrictive cancer cells. We have reported that the activity of cyclin E promoter is enhanced during viral reproduction process $(56,165)$, and virus-induced cyclin $\mathrm{E}$ activates the cyclin $\mathrm{EL}-\mathrm{CDK} 2-\mathrm{pRb} / \mathrm{E} 2 \mathrm{~F}$ pathway to enhance cyclin E expression via a loopback regulation (164). We postulate that oncolytic adenovirus driven by human cyclin E promoter may have a broader range of application since it can be activated in cancer cells with dysregulated cyclin $\mathrm{E}$ and the promoter activity can be further enhanced by virus reproduction.

The mechanism(s) of Ad-cycE induced oncolysis requires further studies. The 
oncolysis mediated by Ad-cycE may correlate with multiple mechanisms such as virus replication, apoptosis, and autophagy. The virus replication and release from cells can directly cause cell lysis as the major mechanism $(7,176)$. Also, studies by our laboratory as well studies by others' have shown that oncolytic Ads with entire $E 1 b$ deletion induce more apoptosis compared to $E 1 b 55 K$-deleted dl1520 and wild-type $\operatorname{Ad}(38,177)$. The expression of E1a gene has been shown to trigger the accumulation of p53 protein, resulting in p53-dependent apoptosis (40, 106). Due to the lack of E1b19K to repress E1A-induced apoptosis, Ad-cycE may induce more apoptosis than dl1520. Additionally, previously we have shown virus replication induces autophagy to promote virus replication and oncolysis (166). Autophagy is a process involving in the lysosomal degradation, recycling of cellular components and type II programmed cell death $(178,179)$. Currently we have studied the mechanism of Ad-cycE-induced oncolysis and are observing Ad-cycE induced LC3-I to LC3-II cleavage and ATG 5-12 complex formation, suggesting that this process may also participate in Ad-cycE-induced oncolysis (Cheng and Zhou, unpublished data).

We extended our findings from in vitro studies to evaluate antitumor effects of Ad-cycE in a human lung cancer xenograft mouse model, which confirmed the potent antitumor efficacy elicited by Ad-cycE in vivo. A long-term survival animal study also allowed us to follow up the responses of individual mice to the Ad-cycE treatment. The tumors in Ad-cycE-treated mice started shrinking at day 9. However, during the long-term follow up (over 100 days) we noticed that each 
mouse had individual response to the Ad-cycE treatment. In Ad-cycE-treated group, two mice had invisible tumor at the end of the study; three mice had repressed tumor growth all the time; two mice had repressed growth for around 20 days but tumors recurred at around 30 days after the first treatment. It should be noticed that if virus treatment cannot completely eliminate the tumor mass, the remnant tumor cells may recur as what may happen in the clinical setting. The variances among the outbred athymic NCr-nu/nu mice may be one important determinant for the fates of tumor repression or recurrence. Our observation pointed out the importance of personalized treatment in the oncolytic virotherapy. Individual response to oncolytic virotherapy should be taken into consideration to develop the strategy to achieve better therapeutic outcomes.

In conclusion, in the present study we have shown that human cyclin E promoter driven Ad-cycE exhibits potent antitumor efficacy in vitro and in vivo. Our study is the first to demonstrate the application of cyclin E promoter-based vector design with dual properties to target the cyclin E overexpression in cancer cells and virus-induced environment. As cyclin $\mathrm{E}$ is highly overexpressed in a variety of cancers and further augmented during virus reproduction, Ad-cycE represents tumor specificity and potential to overcome restriction in nonpermissive cancer cells for d11520. Further studies will help for characterizing the clinical importance of $\mathrm{Ad}-\mathrm{cyc} \mathrm{E}$ in the future cancer treatments. 


\section{CHAPTER IV}

\section{PROPERTIES OF ONCOLYTIC REPLICATION OF ADENOVIRUSES IN MURINE AND HUMAN LUNG CANCER CELLS}

\subsection{Introduction}

Human adenoviruses (Ads) are double-stranded linear DNA viruses. The viral genes are divided into early and late genes relative to the onset of viral gene expression. Ads with deletion of the early $E 1 B 55 K$ gene preferentially replicate in cancer cells and cause oncolysis, leading to the release of amplified and infectious viruses to further destroy tumor cells (5). Studies by our laboratory as well studies by others' have shown that oncolytic Ads can efficiently kill cancer cells in cell culture and strongly inhibit tumor growth in animal experiments (38, 121, 180, 181). E1b55K-deleted ONYX-015 (dl1520) has been applied in clinical trials in the United States and H101 (with the similar structure as ONYX-015) has been commercially approved for cancer therapy in China $(17,18)$. However, the antitumor effects of oncolytic Ads have been somewhat disappointing when used in clinical applications $(182,183)$.

Current animal models used to evaluate the efficacy of oncolytic Ads rely on human tumor xenograft in immunodeficient mouse models. However, human tumor xenograft models are less clinically relevant to the real cancers in patients. 
Human Ads tend to target human tumors and replicate more efficiently in human cells than in murine cells. Thus preclinical results obtained with human tumor xenograft in mouse models may overstate the therapeutic efficacy. Additionally, the lack of functional immune systems in immunodeficient models may hinder the accuracy of clinically predicting the effects in patients $(3,184)$. Ads have complex interactions with host cell immune response effectors $(17,185)$. In the presence of the immune system, the oncolytic effects of the virus may be reduced due to the immune responses against viral particles. Conversely, the immune system may help the tumor-killing effects by recruiting nature killer cells, antibodies, tumor-specific cytotoxic $T$ lymphocytes (CTLs) to enhance the therapeutic outcome (186). Development of immunocompetent mouse models with murine tumor cells in which oncolytic Ads can selectively replicate is necessary and important.

Previously we have demonstrated that cyclin E overexpression is a molecular basis of selective replication of $E 1 b 55 K$-deleted Ads in human cancer cells (56, 79). Ad replication depends on cyclin E induction in cells after Ad infection. E1b55K-deleted Ads fail to efficiently induce cyclin E expression in the normal cells, leading to the restricted replication. However, this E1B55K function is not required in cancer cells which generally already have dysregulated cyclin E; therefore E1b55K-deleted Ads can successfully replicate in cancer cells and cause oncolysis. We recently further reported that Ad-induced cyclin E overexpression adjusts the cellular environment for viral productive replication via 
activating CDK2, that in turn targets the transcriptional repressor $\mathrm{pRb}$ and regulates cell cycle progression (164).

Cyclin $E$ is a nuclear protein essential for the cell cycle progression (80), DNA replication $(110,111)$, and centrosome duplication $(112,113)$. The protein level of cyclin $E$ rises at late $G_{1}$ phase and peaks at the $G_{1} / S$ phase to promote $S$-phase entry in normal cell cycle $(80,81)$. Numerous types of cancers are highly associated with dysregulation of cyclin E, such as cyclin E gene amplification (91), overexpression of mRNA or protein levels $(87,92)$, decrease of cyclin $E$ protein turnover $(93,94)$, or the presence of more active forms of cyclin E (94-96). Constitutive overexpression of cyclin E induces chromosome instability $(89,90)$, impairs normal cell cycle progression and triggers tumor development in transgenic animal models $(86,114,115)$. Human cyclin $\mathrm{E}$ overexpression in mouse lung leads to development of premalignant and malignant lung lesions which resemble to the features found in lung cancer patients $(88,115)$. Murine ED-1 cell line was derived from the lung cancers of cyclin E transgenic mice (187). As oncolytic Ad replication is related to dysregulated cyclin E expression, Ads may selectively replicate in murine ED-1 cancer cells.

Replication of human wild-type Ad5 and oncolytic Ads in murine ED-1 cells was studied and compared with human lung cancer A549 cells which overexpress cyclin $E$. The oncolytic effects of one novel oncolytic Ad-cycE driven by cyclin $E$ promoter (169) on murine ED-1 lung cancer cells was characterized in this study 
along with d11520. Our results showed that human Ads can penetrate ED-1 cells which have the ability to support viral DNA synthesis, early and late gene expression, and virion production. Although with relatively lower production compared to in human cells, the pattern of virus replication in murine ED-1 cells is similar to that in human A549 cells. This study indicates that ED-1 cancer cell line is permissive for human Ad replication and can be used to develop a new immunocompetent murine tumor model for evaluating the therapeutic efficacy of oncolytic Ads. 


\subsection{Materials and Methods}

Cell lines and culture conditions. HEK 293 (ATCC no. CRL-1573), human lung cancer A549 (ATCC no. CCL-185) and mouse embryonic fibroblast NIH/3T3 (ATCC no. CRL-1658) cell lines were purchased from the American Type Culture Collection (Rockville, MD). Murine ED-1 cells line, a lung cancer cell line derived from transgenic mice with wild-type human cyclin $E(88,187)$, was a gift from Dr. Ethan Dmitrovsky and further single-cell subcloned in our laboratory. HEK 293 and A549 cells were cultured in minimal essential medium Alpha. ED-1 cells were cultured in RPMI-1640 medium. All media were supplemented with $10 \%$ fetal bovine serum (FBS) and penicillin/streptomycin (100 U/ml). Cells were cultured in a $5 \% \mathrm{CO}_{2}$ incubator at $37^{\circ} \mathrm{C}$. All cell culture reagents were obtained from Gibco BRL (Bethesda, MD) and Corning Cellgro (Manassas, VA).

Adenoviral vectors. Wild-type adenovirus type 5 (Adwt, ATCC no. VR-5) was used as a replication-competent control. AdCMV/GFP, an Ad vector with E1 deletion carrying a green fluorescent protein (GFP), was used as a replication-defective control. Ad dl1520 is a E1b mutant which contains an 827-bp deletion and a point mutation to generate a premature stop codon in the E1B55K coding region (15). Ad-cycE is a novel E1b-deleted oncolytic vector carrying a human cyclin $\mathrm{E}$ promoter driving an intact $\mathrm{E} 1 \mathrm{~A}$ expression cassette. The endogenous E1a promoter was deleted and a human cyclin E promoter (GenBank ID: X95406) was inserted to replace the deleted E1a promoter in Ad-cycE. Therefore, Ad-cycE contains a human cyclin E promoter to control E1a 
open reading frames (ORF) (169). All of the vectors created and used in this study are based on the backbone of wild-type Ad type 5 .

MTT assay. Cells were seeded into 24-well plates at a density of $2.5 \times 10^{4}$ (cells/well) and cultured under the indicated conditions. Cell proliferation was assessed at three days after respective treatments by measuring the conversion of the 3-(4,5-dimethylthiazol-2-yl)-2,5-diphenyltetrazolium bromide (MTT) to purple formazan, according to the manufacturer's instructions (Boehringer Mannheim, Indianapolis, IN). The absorbance of the supernatant was measured at $590 \mathrm{~nm}$ using a Synergy HT Multi-Mode Microplate Reader (Bio-Tek, Winooski, VT). The results were expressed as the fold change relative to the result at day 0 . Doubling time was analyzed from the curves of cell growth in log phase with the exponential regression analysis provided by http://www.doubling-time.com (188-190).

Cytotoxicity assay. Cells were seeded into 24-well plates at a density of $2.5 \times 10^{4}$ (cells/well) and cultured under the indicated conditions. Cytotoxicity was assessed with crystal violet staining (170). Cells were fixed with $3.7 \%$ paraformaldehyde and stained with $1 \%$ crystal violet followed by washing with water to remove excess dye. The dye was solubilized with $2 \%$ SDS and the absorbance of the solubilized stain was measured at $590 \mathrm{~nm}$ using a Synergy HT Multi-Mode Microplate Reader (Bio-Tek, Winooski, VT). The OD values were quantitated into the cell viability $\%$ by the formula, cell viability $\%=(O D$ value of 
experimental group / OD value of control group) $\times 100 \%$. The mock-control group was calculated as $100 \%$ of cell viability in the assay (171).

Southern blot analysis. After viral infection, cells were collected at different time points. The viral DNA synthesis was determined with Southern blot; $1 \mu \mathrm{g}$ of isolated genomic DNA was digested with the restriction enzyme Pstl and analyzed with $1 \%$ agarose gel, which was subsequently transblotted to a Hybond-N+ membrane (YA3609; Amersham Pharmacia Biotech, Arlington Heights, IL). The probe was prepared by digesting $0.5 \mu \mathrm{g}$ pBHGE3 (122) with Pstl and labeled by following the protocol of Amersham AlkPhos Direct Labeling and Detection Systems (RPN 3690; GE Healthcare, Piscataway, NJ). The blot was prehybridized for $3 \mathrm{hrs}$ at $63^{\circ} \mathrm{C}$. The hybridization and stringency washes were performed at $60^{\circ} \mathrm{C}$ and followed by the chemiluminescent detection according to the manufacturer's protocol. Densitometric value for the bands was quantified by Gel-pro Analyzer 4.0 software (Media Cybernetics, Bethesda, MD) (191) and expressed as integrated optical density (I.O.D.).

Western blot analysis. Infected cells were harvested at indicated time points and lysed with CDK2 lysis buffer (20 mM Tris $\mathrm{pH} 7.5,150 \mathrm{mM} \mathrm{NaCl}, 5 \mathrm{mM} \mathrm{MgCl}$, $0.5 \%$ Nonidet P-40, 0.1\% Brij 35, 5 mM sodium glycerophosphate, $1 \mathrm{mM}$ sodium vanadate, $1 \mathrm{mM}$ dithiothreitol). The Western blot analyses were performed as described previously (123). Briefly, $60 \mu \mathrm{g}$ of cell lysates were electrophoresed through $12 \%$ SDS-polyacrylamide gels and transferred onto an Immobilon-P 
Membrane (Millipore, Billerica, MA). The primary antibodies used in this study were rabbit anti-cyclin E (M-20), (Santa Cruz Biotechnology, Santa Cruz, CA), mouse anti-adenovirus type $5 \mathrm{E} 1 \mathrm{~A}$ (BD Pharmingen, San Jose, CA), and rabbit anti-adenovirus type 5 antibody (Abcam, Cambridge, MA). Actin was used as an internal control. The membranes were then incubated with anti-mouse immunoglobulin $G$ ( $\lg G$ ) or anti-rabbit IgG peroxidase-linked species-specific whole antibody (GE Healthcare, Piscataway, NJ). Chemiluminescent detection was performed with ECL reagents according to the supplier's recommendations (GE Healthcare).

Viral titration. Total infected cells and culture supernatants were collected at the indicated time points and lysed to release virus particles with three cycles of freezing and thawing. The viral titers were determined by the infective unit method as described previously $(120,121)$. Briefly, HEK 293 cells were seeded in 96-well plates at a density of $10^{3}$ (cells/well) and then infected with 10-fold serially diluted viruses. CPE was recorded and scored after incubation for 7 days.

Burst assay. Burst assay were used to determine the replication efficiency of human Ads in infected cells (192-194). Cells were seeded in 6-well plates at a density of $2 \times 10^{5}$ (cells/well) for $4 \mathrm{hr}$ and infected with human Ads at 3.5 (for A549 cells) or $10 \mathrm{MOI}$ (for ED-1 cells). At $18 \mathrm{hr}$ postinfection (p.i.) cell supernatants were removed and the cell monolayers were washed twice with PBS. At $18 \mathrm{hr}$ and $120 \mathrm{hr}$ p.i., cells and supernatants were collected. The viral titers were determined 
by the infective unit method. The burst ratio was expressed as the titer of virus at $120 \mathrm{hr}$ p.i. (virus output) relative to the titer of virus at $18 \mathrm{hr}$ p.i. (virus input). An increase ratio in virus titer after 120 hours indicates virus replication.

Statistical analyses. All above experiments, except specifically indicated, were repeated at least three times. Quantitation results were reported as means \pm standard deviation (S.D.). Statistical difference of the combination experiment was assessed with a Student's $t$-test. Statistical significance of difference was set at $p$ $<0.05$. 


\subsection{Results}

\section{Murine ED-1 cells show higher growth rate and lower serum sensitivity than}

human A549 cells. Human A549 and murine ED-1 lung cancer cell lines both have well-known backgrounds of the cyclin E expression. A549 cell line is the human lung cancer cell line with constitutive cyclin E expression and has been reported as a highly permissive cancer cell line for Ad oncolytic replication (56). ED-1 cell line was derived from transgenic mice with wild-type human cyclin E expression in lung cancers $(88,187)$. The growth characteristics of two cell lines were first compared in this study. Cells were cultured under 10\% FBS and the cell proliferation of the two cell lines were determined by MTT assay. The final number of ED-1 cells increased 10 -fold from day 0 to day 3, while A549 cells increased 5.6-fold when cultured with 10\% FBS (Fig. 4.1A). The doubling time generated from the data of cell growth in log phage indicated that the doubling time of ED-1 cells is $18.6 \mathrm{hr}$ and A549 is $30.17 \mathrm{hr}$, showing the growth rate of ED-1 cells is about 1.6-fold greater than that of A549 cells. Growth curves of A549 and ED-1 cells in the presence of serum concentrations ranging from $0 \%$ to $10 \%$ were shown in Figure 3.1B. The results showed that proliferation of both cell lines were repressed under lower serum concentrations. The number of A549 cells cultured in medium with $0 \%$ serum only increased slightly, however ED-1 cells still managed to increase close to 4 fold under the same conditions (Fig. 4.1B). Thus, ED-1 cells grow significantly faster and exhibit less sensitivity to the decreased serum concentration than A549 cells. 
A.

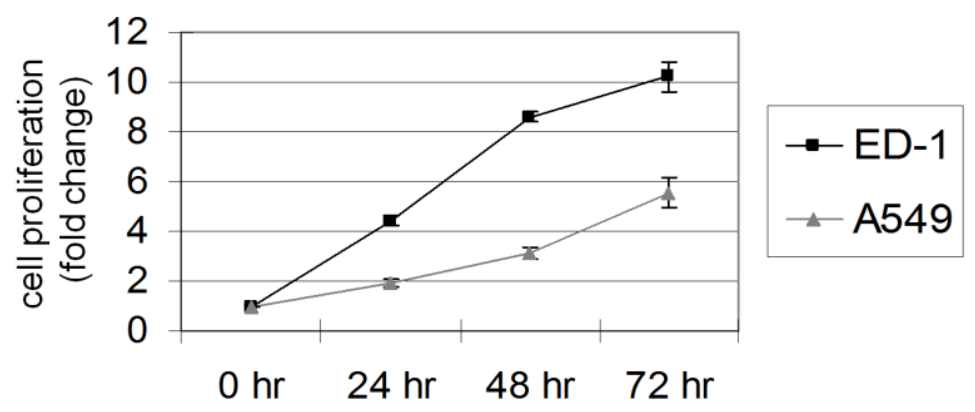

B.
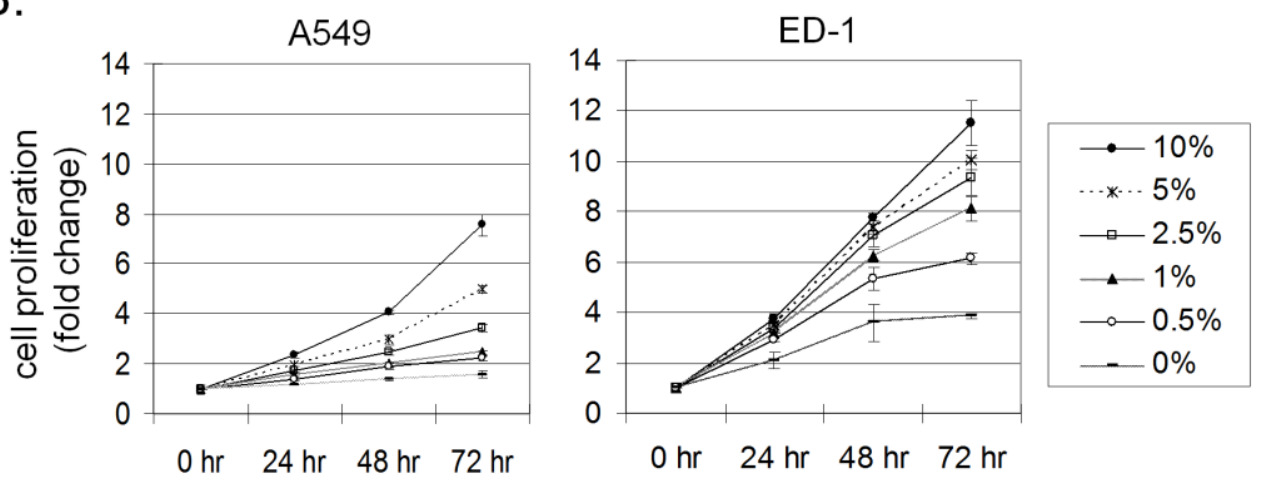

Figure 4.1. Growth characteristics of A549 and ED-1 cells. Cell proliferation and serum sensitivity of A549 and ED-1 cells were determined by MTT assay at 0 , 24, 48 and $72 \mathrm{hr}$. Cells were seeded into 24 -well plates at a density of $2.5 \times 10^{4}$ (cells/well) and cultured in (A) $10 \%$ FBS or (B) $0,0.5,1,2.5,5$ and $10 \%$ FBS, respectively. The results were expressed as the fold change relative to the result at $0 \mathrm{hr}$. All values represent the means \pm S.D. of independent triplicate. 


\section{ED-1 cells require 3-fold higher titers of Ads to achieve similar infection}

efficiency as A549 cells. To maintain cells growth under a similar rate and density, A549 and ED-1 cells were cultured in 1\% FBS and 0\% FBS, respectively. Two approaches were applied to evaluate the infection efficiency of human Ads in A549 and ED-1 cells. First, we evaluated the Ad infection efficiency by quantifying the number of cells expressing GFP using a vector expressing a GFP reporter gene (AdGFP). A549 and ED-1 cells were infected with increasing MOI of AdGFP after seeding for $4 \mathrm{hr}$. For each infection, three random fields were taken by EVOS fluorescence microscope (AMG, Bothell, WA) at $72 \mathrm{hr}$ p.i. (Fig. 4.2A). The number of GFP positive cells and the total cell numbers on each photo were counted with ImageJ (US National Institutes of Health, Bethesda, MD). The numbers of GFP positive cells were divided by total cell numbers on each photo to determine the infection efficiencies and presented in Figure 4.2B. Maximum infection of A549 cells (90\%) can be achieved by Ads with an MOI of 10, but ED-1 cells are required at least an $\mathrm{MOI}$ of 20 . To have about $60 \%$ cell infected, an $\mathrm{MOI}$ of 10 is required for ED-1 cells and an MOI of 2.3 for A549 cells (Fig. 4.2B). 
A.

A549 0
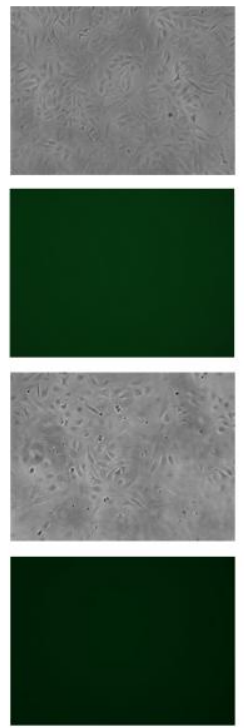

ED-1 0
1.25
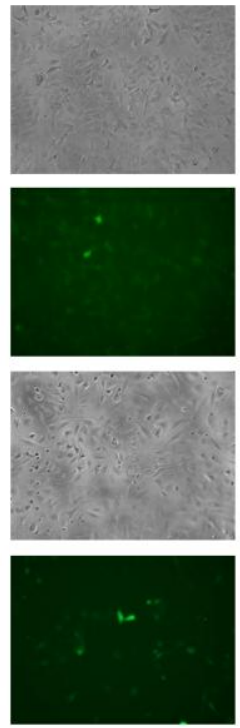

1.25
2.5
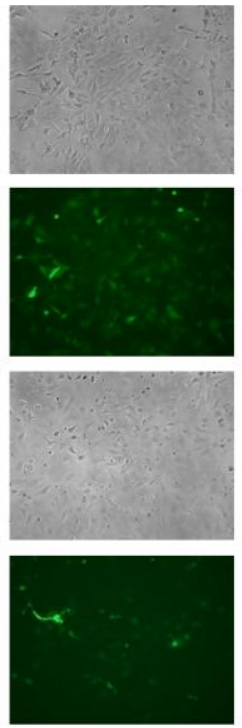

2.5
5
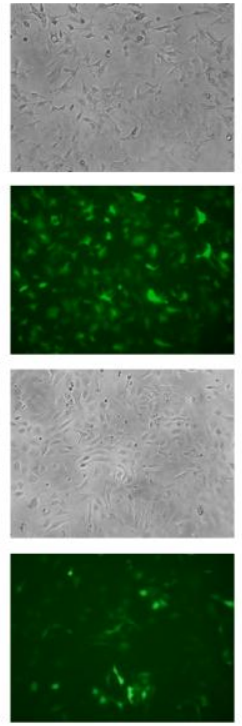

5
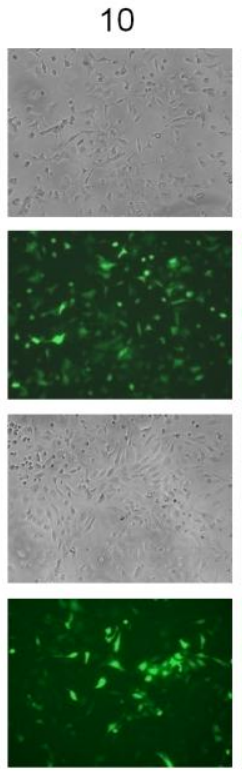

10

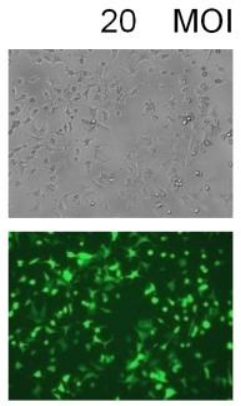

B.

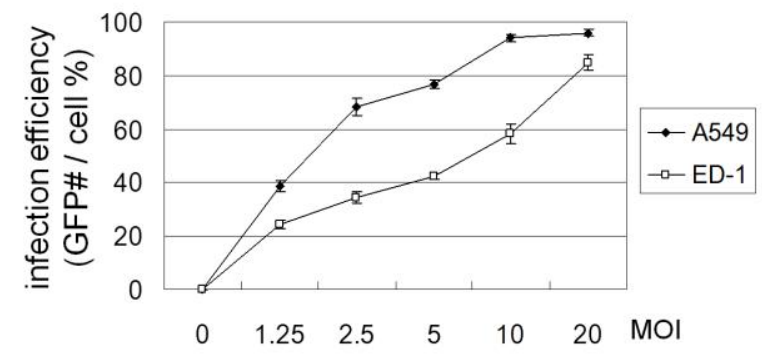

Figure 4.2. Infection efficiency of human adenoviruses on A549 and ED-1 cells. A549 cells were cultured with $1 \%$ FBS and ED-1 cells were cultured with $0 \%$ FBS at a density of $10^{5}$ (cells/well). (A) The photographs were taken by EVOS fluorescence microscope (AMG, Bothell, WA) at $72 \mathrm{hr}$ p.i. (B) The numbers of GFP cells on each photo were counted with ImageJ (US National Institutes of Health, Bethesda, MD). The results were expressed as GFP cell numbers relative to total cell numbers on the scene. All values represent the means \pm S.D. of independent triplicate. 
Secondly, considering that promoter activity and GFP expression may differ from cell lines and interfere in determining the infection efficiency, we also used Southern blot to evaluate the infection efficiency by quantitating the amount of $\mathrm{Ad}$ DNA. As AdGFP is a non-replicative virus, the amounts of AdGFP DNA inside the cells should represent the amounts of virus DNA that entered into those cells initially. A549 and ED-1 cells were infected with AdGFP at increasing MOI. Cells were then harvested at $12 \mathrm{hr}$ p.i. and the total DNA was isolated. The DNA samples were digested with Pstl and then analyzed by Southern blot with Ad DNA fragments. Figure 4.3A represents the result of human AdGFP DNA entering ED-1 and A549 cells. We observed that with the same MOI of AdGFP infection, Ad DNA amount entering ED-1 cells was lower than that entering A549 cells. Yet increasing infection $\mathrm{MOI}$ of AdGFP led to a concomitant increase of Ad DNA amount in both ED-1 and A549 cells, suggesting virus entry can be adjusted by altering the infection MOI of AdGFP. To compare the concentration of Ad DNA in ED-1 and A549 cells, we specifically determined the viral DNA densities in ED-1 cells infected with $10 \mathrm{MOI}$ and in A549 cells infected with 2.5, 5 and $10 \mathrm{MOI}$ (boxed in Fig. 3A). Densitometric values for the bands were quantified by Gel-pro Analyzer 4.0 software (Media Cybernetics, Bethesda, MD) (191) and expressed as integrated optical density (I.O.D.) (Fig. 4.3B). The algorithmic result revealed that to achieve a similar infection with an MOI of 10 AdGFP for ED-1 cells, an MOI of 3.5 is required for A549 cells. In the following experiments, we chose $3.5 \mathrm{MOI}$ of human Ad to infect human A549 cells and $10 \mathrm{MOI}$ human Ad to infect murine ED-1 cells to achieve similar virus infection level for future experiments. 
A.

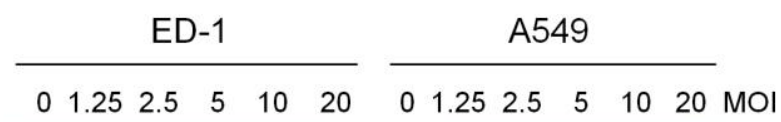

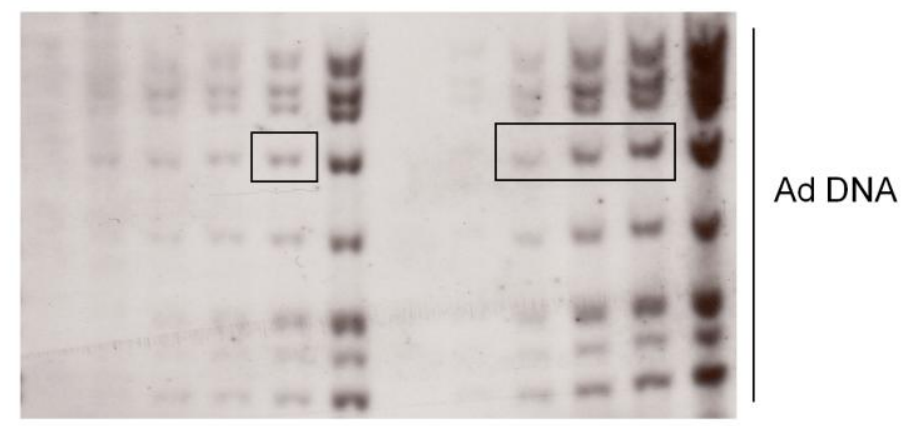

B.

\begin{tabular}{|c|c|c|c|c|c|}
\hline & ED-1 & & A549 & & \\
\hline & 10 & 2.5 & 5 & 10 & $\mathrm{MOI}$ \\
\hline & & $=0$ & $\omega$ & $=$ & \\
\hline $\begin{array}{l}\text { Band } \\
\text { density } \\
\text { (IOD) }\end{array}$ & 84.4 & 35.3 & 218.5 & 384.7 & \\
\hline
\end{tabular}

Figure 4.3. The DNA amounts of AdGFP entering cells. (A) A549 cells were cultured with $1 \%$ FBS and ED-1 cells were cultured with $0 \%$ FBS at a density of $10^{6}$ (cells/well) in $60 \mathrm{~mm}$ dishes. Cells were infected with AdGFP at 0, 1.25, 2.5, 5, 10 and $20 \mathrm{MOI}$, respectively, and harvested at $24 \mathrm{hr}$ p.i.. The DNA isolated from cells was totally loaded into the agarose gel. The amounts of AdGFP entering cells were quantitated by analyzing virus DNA amounts in cells with Southern blot. (B) Densitometric value for the bands was quantified by Gel-pro Analyzer 4.0 software (Media Cybernetics, Bethesda, MD) and presented as integrated optical density (I.O.D.) values. 
Human oncolytic Ads are able to replicate in both murine and human lung cancer cell lines but poorly in noncancerous murine cell line. Murine $\mathrm{NIH} / 3 \mathrm{~T} 3$ cells generated from NIH Swiss mouse embryo fibroblasts (195) were applied here as a noncancerous control to compare with murine ED-1 lung cancer cell line. Relatively high levels of cyclin E expression was detected in human A549 and murine ED-1 lung cancer cells but not in NIH/3T3 cells (Fig. 4.4A). The cytotoxicity of human Ads on cells were photographed and quantitated. AdGFP with deletion of $E 1$ region was used as a non-replicative control. Replication-competent wild-type Ad5 (Adwt) and oncolytic dl1520 (ONYX-015) and Ad-cycE were chosen to evaluate the human Ad replication. Ad d11520 is an attenuated adenovirus with E1b deletion which has been evaluated in several clinical studies $(15,17)$. Ad-cycE is an E1b-deleted oncolytic vector carrying a human cyclin $\mathrm{E}$ promoter driving $\mathrm{E} 1 \mathrm{~A}$ transcription to target lung cancers with cyclin E overexpression (169). To achieve equal infectivity, human A549 was mocked-infected or infected with AdGFP, dl1520 and Ad-cycE at 3.5 MOI and both murine ED-1 and NIH/3T3 cells were infected with various viruses at $10 \mathrm{MOI}$. The CPE became visible at 48-72 $\mathrm{hr}$ p.i., showing morphological change as infected cells rounded up and detached from the cell monolayer. Cytotoxicity or cell CPE caused by Ad infection was photographed with an inverted microscope (Olympus CKX41) (Fig. 4.4B). Mock-infection and non-replicative AdGFP infection did not induce cytotoxicity in cells. Adwt induced cytotoxicity in all cell lines with non-selectivity; however the two oncolytic viruses dl1520 and Ad-cycE only induced observed cytotoxicity on both A549 and ED-1 lung caner cells but 
not on noncancerous $\mathrm{NIH} / 3 \mathrm{~T} 3$ cells. Cell viability was determined by crystal violet staining and then quantified by comparing each group to the mock-infected group (Fig. 4.4C). The results matched to the observations of virus CPE, suggesting the selectivity of oncolytic Ads existing in murine cells. 
A.

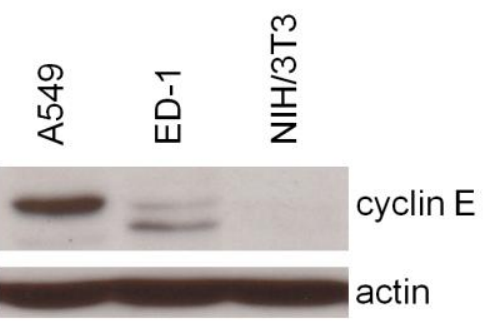

B.

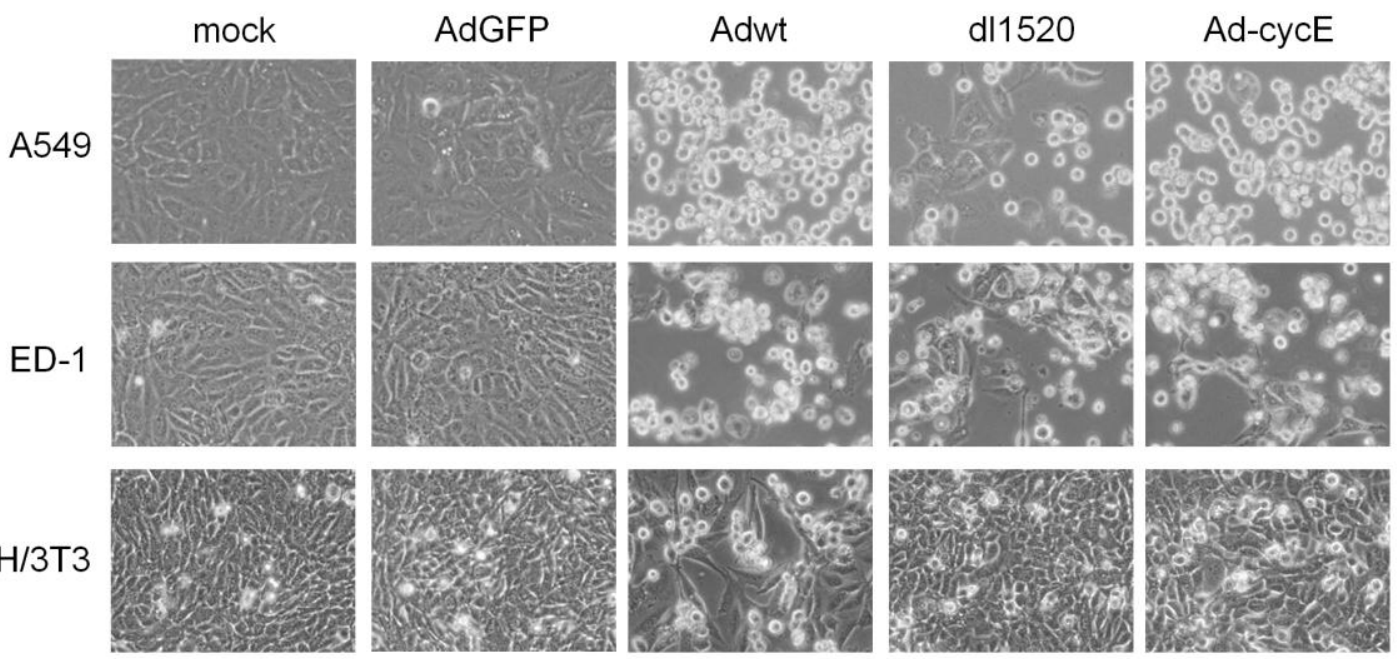

C.

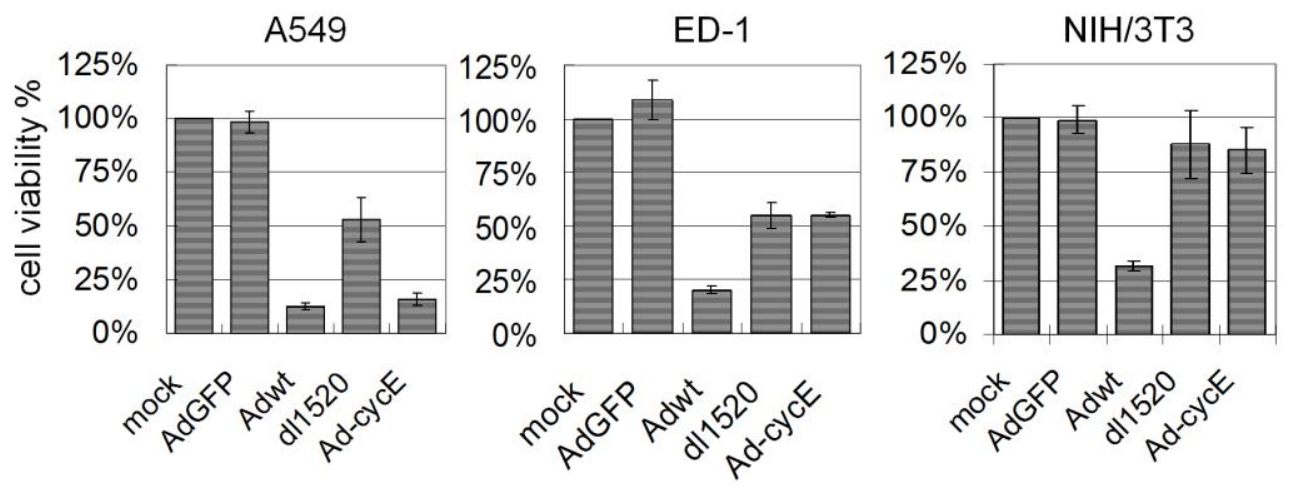


Figure 4.4. Cyclin E expression and cytopathic effects of human Ads on human A549, murine ED-1, and NIH/3T3 cells. (A) Cells were seeded in $60 \mathrm{~mm}$ dishes at a density of $10^{6}$ for $18 \mathrm{hr}$ and then collected. The cell lysates were immunoblotted for cyclin E protein and actin. Actin was used as a loading control. (B) Cells were mock-infected or infected with AdGFP, Adwt, dl1520 or Ad-cycE at 3.5 $\mathrm{MOI}$ (for A549 cells) or $10 \mathrm{MOI}$ (for ED-1 and NIH/3T3 cells). CPE was observed at $72 \mathrm{hr}$ p.i and photographed with an inverted microscope Olympus CKX41. (C) The cell viability \% was determined and the values represent the means \pm S.D. of independent triplicate compared with the mock-infected group. 
To determine whether the cytotoxicity was induced by complete virus replication in murine cells, burst assay was used to compare the virus output with virus input (192-194). Normally the burst ratio is generated by comparing virus production at $72 \mathrm{hr}$ (final virus output) to $4 \mathrm{hr}$ (initial virus amount entering cells) and an increased ratio indicates virus replication. Considering human Ad infection for $4 \mathrm{hr}$ caused very low infection efficiency in murine ED-1 cells, we chose $18 \mathrm{hr}$ as the initial point and chose $120 \mathrm{hr}$ at which the infected cells showed up complete CPE as the end point. Yields of Adwt, dl1520 and Ad-cycE increased 756-, 474-, and 269-fold on average after infection in ED-1 cells, respectively. However, Ad titers only increased 25 fold for Adwt and 2 fold for dl1520 along with Ad-cycE in $\mathrm{NIH} / 3 \mathrm{~T} 3$ cells (Fig. 4.5). The burst ratio of Adwt in ED-1 is 30 times greater than that in $\mathrm{NIH} / 3 \mathrm{~T} 3$, and the burst ratio of oncolytic Ads were 100-200 fold increase than that in $\mathrm{NIH} / 3 \mathrm{~T} 3$, indicating ED-1 is more permissive for human Ad replication than $\mathrm{NIH} / 3 \mathrm{~T} 3$. 

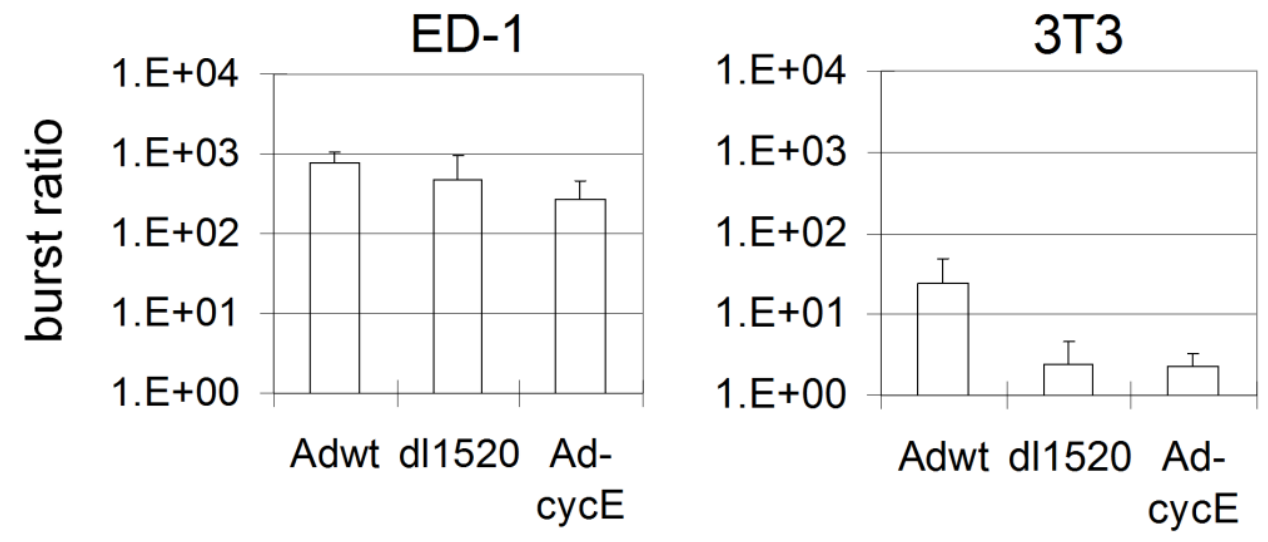

Figure. 4.5. Burst ratios of human Ad replication in ED-1 and NIH/3T3 cells. Cells were infected with Adwt, dl1520, and Ad-cycE at $10 \mathrm{MOI}$ for $18 \mathrm{hr}$ or $120 \mathrm{hr}$. The virus yields were determine by infection unit method and expressed as burst ratios, representing virus yields at $120 \mathrm{hr}$ p.i. relative to virus yields at $18 \mathrm{hr}$ p.i.. The values represent the means \pm S.D. of independent triplicate. 
Wild-type and oncolytic human Ads replicate in both human and murine lung cancer cells. To further characterize the properties of human Ad replication in A549 and ED-1 cells, Ad DNA synthesis, E1A expression, the production of viral capsid proteins and the virus yields were analyzed. The viral DNA synthesis of human Ad was determined by Southern blot with the probes of Ad genomic DNA. As shown in Figure 4.6, mock-infected group did not contain viral DNA and the viral DNA level of non-replicative AdGFP was not detectable on the blot. Only in replication-competent Ad groups we detected viral DNA level which continuously increased from $24 \mathrm{hr}$ to $48 \mathrm{hr}$ p.i.. The results showed that Adwt, dl1520 and Ad-cycE can replicate in both human and murine lung cancer cells; oncolytic Ad-cycE can achieve the similar level as d11520. The viral DNA synthesis level in ED-1 is lower than that in A549. 


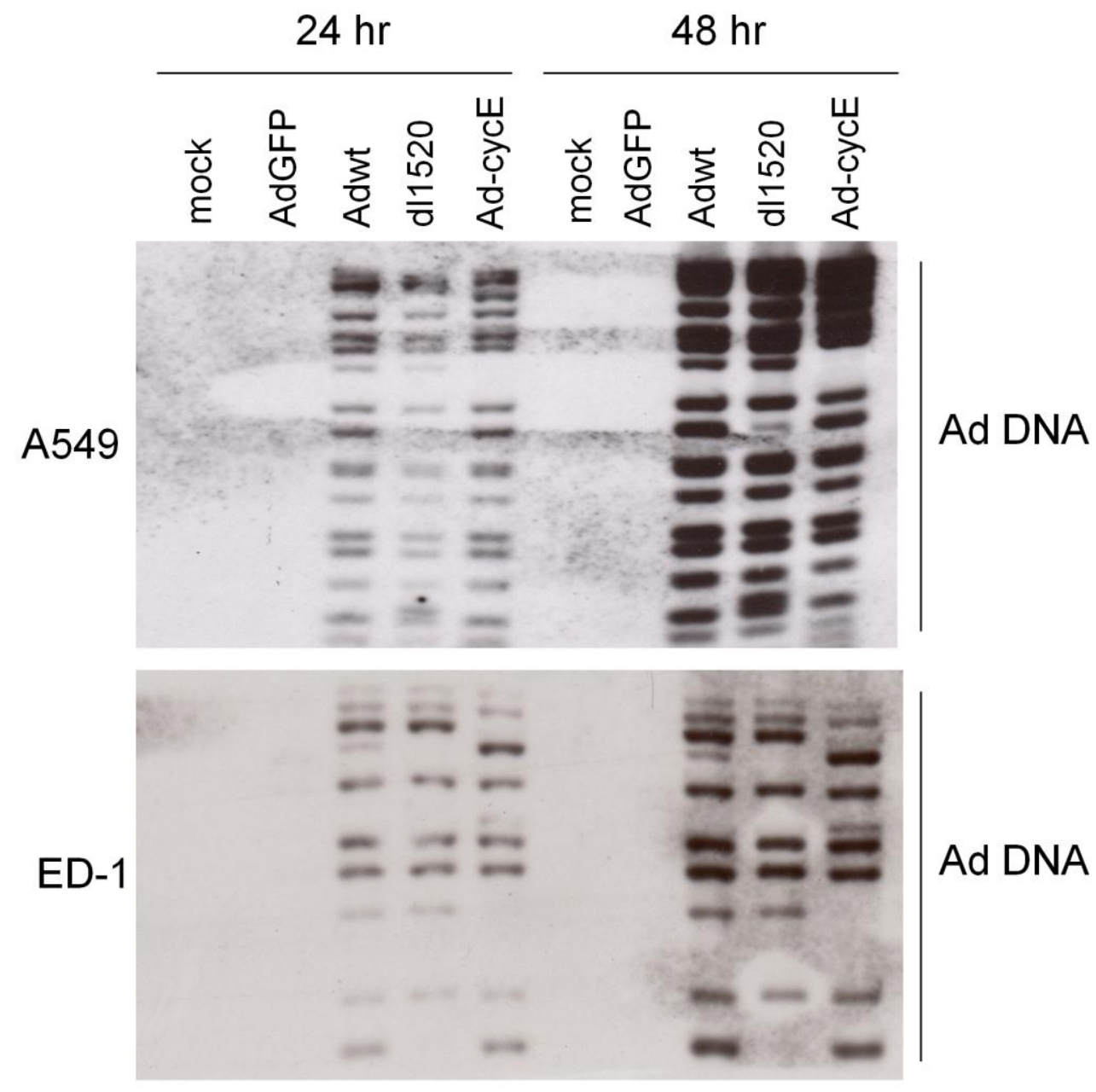

Figure. 4.6. Human Ad DNA synthesis in A549 and ED-1 cells. Virus DNA synthesis was determined by Southern blot. $3.5 \mathrm{MOI}$ was used to infect A549 cells and $10 \mathrm{MOI}$ was used to infect ED-1 cells to achieve the similar infection efficiency. The DNA was isolated from the cells at 24 and $48 \mathrm{hr}$ p.i. and fragmented with the restriction enzyme Pstl. The probe was Adwt genome DNA. 
The E1A expression level was examined by Western blot at $24 \mathrm{hr}$ p.i. E1A is the crucial protein which is expressed immediately after infection and initiates the virus replication cycle (37). Ad E1A protein expression was examined at $24 \mathrm{hr}$ and identified as multiple bands in the range of $35-46 \mathrm{kDa}$ generated from the alternative splicing of E1A transcripts (196). As the pattern shown in human A549 cells and murine ED1 cells, Ad E1A expression was only detected in the groups infected with replication-competent Adwt, dl1520 and Ad-cycE, but not in the groups mock-infected or infected with non-replicative AdGFP carrying the deletion of $E 1$ region (Fig. $4.7 A)$.

The capsid protein production of human Ads in A549 and ED-1 was shown in Figure 4.7B. Consistent to the pattern of E1A expression, capsid protein production at $72 \mathrm{hr}$ was detected in both human and murine cancer cells infected with replication-complete Adwt, dl1520 and Ad-cycE. To determine the virus production in both cell lines, virus yields were determined by the infection unit method and monitored over the time. Figure 4.7C revealed that virus yields of human Ads in murine ED-1 cells and human A549 cells increased over the time; the overall virus yield in ED-1 cells was 10-fold lower than that in human A549 cells. The titers of Adwt, dl1520 and Ad-cycE produced by A549 cell culture at 72 $\mathrm{hr}$ after infection increased to the range between $10^{8}$ and $10^{9}$ (IFU/ml); while the virus titers produced by ED-1 were between $10^{7}$ and $10^{8}$ (IFU/ml) (Fig. 4.7C). Taken all data together, we demonstrated that wild-type and oncolytic dl1520 and Ad-cycE can replicate in both human A549 and murine ED-1 lung cancer cells. 
A.

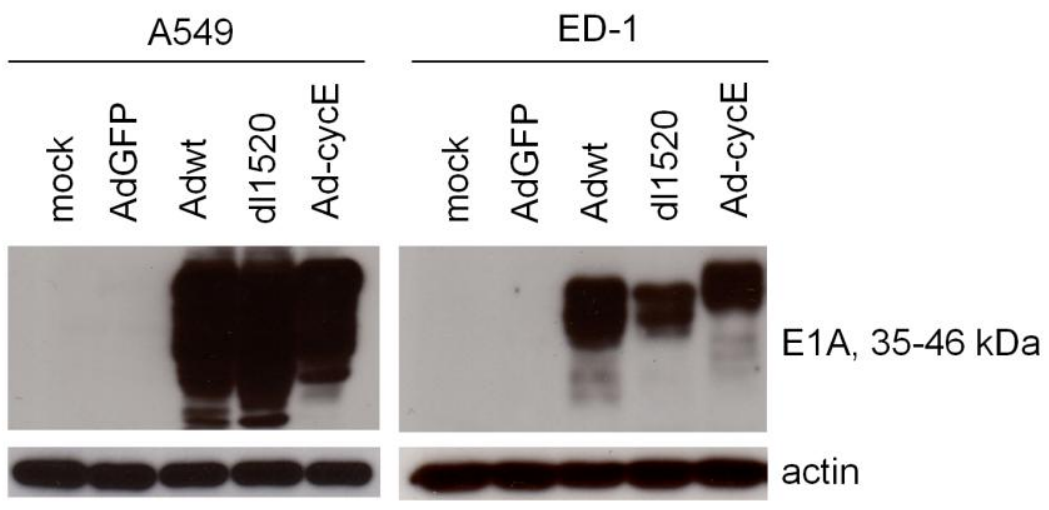

B.
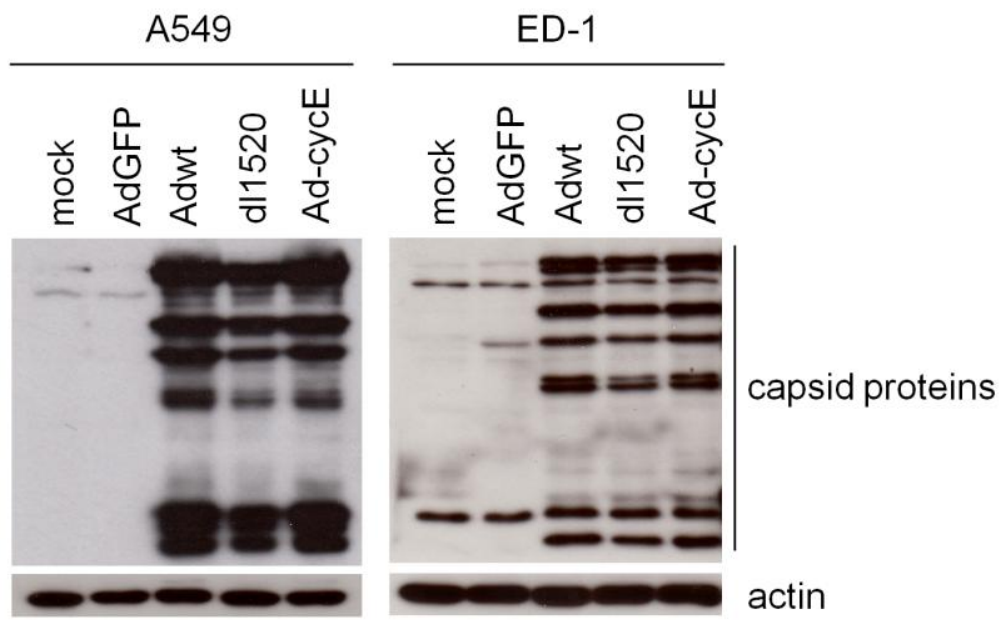

C.
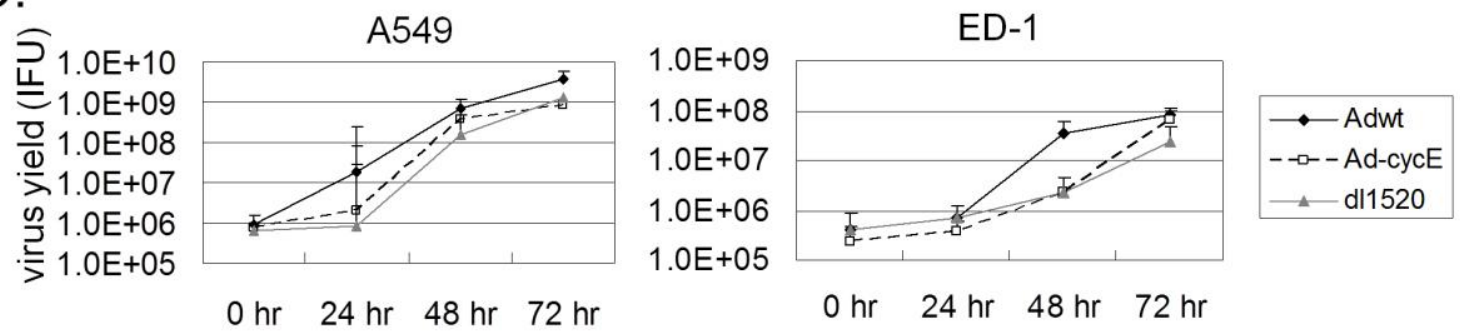
Figure 4.7. The expression of viral E1A, capsid protein and virus production in A549 and ED-1 cells. (A) Cells were mock-infected or infected with AdGFP, Adwt, dl1520 or Ad-cycE at $3.5 \mathrm{MOI}$ (for A549 cells) or $10 \mathrm{MOI}$ (for ED-1). Cells were collected at $24 \mathrm{hr}$ p.i. and the cell lysates were immunoblotted for E1A protein and actin. (B) Infected cells were collected at $72 \mathrm{hr}$ and the cell lysates were immunoblotted for viral capsid protein and actin. Actin was used as a loading control. (C) The virus yields were determined at $0,24,48$, and $72 \mathrm{hr}$ p.i. with the infection unit method. The values represent the means \pm S.D. of independent triplicate. 


\subsection{Discussion}

Adenovirus-mediated oncolysis is a novel approach for cancer therapy. Current animal experiments rely on immunodeficient mouse models with human xenograft tumors to evaluate the efficacy of oncolytic Ads. Due to the species specificity, human Ads tend to infect and replicate in human tumors rather than in murine tissues. Thus preclinical results obtained with human tumor xenografts in immunodeficient mouse models may overstate the therapeutic effects of oncolytic Ads. In addition, the patient's immune responses may inhibit or augment oncolytic therapy. In this study, we compared Ad replication in murine and human lung cancer cells. We found that human Ads can penetrate and replicate in both murine and human lung cancer cells. When murine and human cancer cells are equally infected, viral DNA synthesis, early and late gene expression and virion production are observed with similar effects in both human and murine cells. Although with a lower production compared to human cells, human Ads can selectively replicate in and destroy murine cancer cells.

Murine cells require a higher $\mathrm{MOI}$ of Ads to achieve same level infection as with human cancer cells. Entry of Ads into cells includes two well-known steps. The first step initiates from the high-affinity binding of adenovirus fiber knob to the coxsackievirus and adenovirus receptor (CAR) (197-200), followed by secondary interaction of the penton base with cell surface integrins to mediate the internalization of virus $(201,202)$. Human CAR is a transmembrane protein belonging to the immunoglobulin superfamily (203). The primary amino acid 
sequences of human and murine CARs are highly homologous (203), thus the murine CAR may function as the key receptor for human Ad to enter murine cells. Besides murine CAR, cell surface integrins may be the other mediator for human Ad entry into the murine cells. Integrins are a family of cell-surface receptors for adhesion to extracellular matrix proteins or counter receptors on other cells (204). The RGD motif of the human Ad penton base serves as a recognition site for several human cellular integrins including $\alpha \mathrm{v} \beta 3, \alpha \mathrm{v} \beta 5, \alpha \mathrm{v} \beta 1$ and $\alpha 3 \beta 1$ (197, $201,204,205)$. Studies have shown that some murine integrins share high homology with human integrins $(206,207)$. Additionally, heparan sulphate glycosaminoglycans (HS-GAGs) may play an important role in the cross-species infection of human Ad on murine cells. HS-GAGs have been shown to mediate CAR-independent attachment and infection by human Ads 2 and 5 in previous reports $(208,209)$. The basic motif of Lys-Lys-Thr-Lys (KKTK) on human Ad fiber shaft near the penton base was found to bind HS-GAGs $(197,210,211)$. Raman et al. indicated that instead of murine CAR, mouse adenovirus type 1 utilizes cellular $\alpha \mathrm{v}$ integrin and HS-GAGs as the primary receptors to enter murine cells (211). The infection of human Ads on murine ED-1 cells can be mediated via the known murine CAR, coreceptors such as integrins and heparan sulfate glycosaminoglycans or even by the other unknown pathways.

Determinants of human Ad replication are also related to other steps of interaction with host cells. Once the human Ad particles are internalized into cells, viral replication in murine ED-1 lung cancer cells proceeds as in human A549 lung 
cancer cells. We also compared human Ad replication in murine cancer cells and in noncancerous murine $\mathrm{NIH} / 3 \mathrm{~T} 3$ cells. Oncolytic Ads virions were produced at high levels in cancer ED-1 cells, but not in NIH/3T3 cells. Obviously, the entire human Ad life cycle can be completed in murine ED-1 lung cancer cells, but it is repressed in $\mathrm{NIH} / 3 \mathrm{~T} 3$ cells. It has been reported that human Ads normally undergo abortive replication but still can cause cell lysis in murine cells $(212,213)$. Virus yields of Ad2 and Ad5 in 3T3-Swiss and BALB/c $3 T 3$ cells were reduced 3 to 5 logs compared to virus production in human cells $(214,215)$, and no infectious virus particles of $A d 12$ were detected in murine $3 T 3$ (embryo) and $L$ (connective tissue) cells $(213,216)$. The block of human Ad replication in murine cells may be related to the low infectivity, unstable and reduced DNA synthesis, abortive expression of late proteins or the defective assembly and maturation at an later stage of viral replication cycle (213-218). Similar phenomenon was observed when we studied the human Ad replication in Chinese hamster ovary $(\mathrm{CHO})$ cells. Even though we continuously observed that human wild-type Ad induces cytotoxicity on $\mathrm{CHO}$ cells, lack of late protein production appears to prevent infectious virion production in $\mathrm{CHO}$ cells (Cheng and Zhou, unpublished data). Our study shows that murine cancer ED-1 cells, but not noncancerous NIH/3T3, have the properties to allow human Ads to complete reproduction steps.

While the exact mechanism(s) supporting the complete reproduction process of human Ads in murine cancer ED-1 cells remains to be investigated, it is tempting to speculate that the human cyclin E overexpression in the cell line may play an 
important role. Cyclin E expression in murine ED-1 cells may support the virus reproduction process on multiple ways. Under the normal condition, cyclin E regulates cell cycle progression, DNA replication licensing $(110,111,219)$, centrosome duplication $(112,113)$ and E2F activation (85). We previously demonstrated that human Ad replication relies on cyclin $\mathrm{E}$ induction by $\mathrm{Ad}$ E1B55K (56). Ad-induced cyclin E turns on the pRb/E2F pathways by activating CDK2 (164). It is possible that human Ad replication in murine ED-1 cells can be enhanced by cyclin E overexpression and/or any of cell proliferation alterations occurring in the carcinogenesis caused by the cyclin E overexpression. Potentially, ED-1 cells may be important to clarify the factors altered by cyclin E overexpression that allow cross-species replication of human Ads.

In this report, we have shown that oncolytic Ads can selectively replicate in and destroy murine ED-1 cancer cells derived from the mouse lung adenocarcinomas. Currently we have inoculated FVB mice with ED-1 cells and are observing the formation of syngeneic mouse tumors in the animals. This study indicates that with murine cancer cells permissive for oncolytic Ad replication, it is feasible to establish syngeneic and immunocompetent mouse tumor models for preclinical studies that will better evaluate the anticancer effects of oncolytic Ads and predict therapeutic outcome in the clinical setting. 


\section{CHAPTER V}

\section{COMBINATION OF AUTOPHAGY INDUCER RAPAMYCIN AND ONCOLYTIC ADENOVIRUS IMPROVES ANTITUMOR EFFECT IN CANCER CELLS}

\subsection{Introduction}

Oncolytic virotherapy with E1b55K-deleted adenoviruses (Ads) has been applied to human clinical trials in the United States and approved for the commercial use in China $(18,27,32,180,220)$. The selective oncolytic effects can be achieved by a small quantity of viruses that spread to surrounding tumor cells, therefore contributing to an interesting drug platform $(4,153)$. Considering the viral tropism, respiratory oncolytic Ads should have a high potential for lung cancer therapy (221). However, lung cancer is generally difficult to treat with oncolytic viruses, and there are few recorded successful trials due to the cancer's propensity to metastasize and the irregular shape of most tumors. Therefore, developing combination strategies to target human lung cancer with improved oncolytic Ads would allow for more effective treatment.

In clinical treatments, oncolytic Ads are generally used with the first-line chemotherapy drugs, and the combination treatments have exhibited high therapeutic efficiency and improved safety (222). However, the interaction mechanism between chemotherapy drugs and viruses has not been well charac- 
terized. Selecting drugs for combination therapies based on the understanding of the interaction between Ads and drugs definitely will benefit the feasibility of this strategy. In our previous study, we have shown that the treatment of the autophagy inducer rapamycin increased the Ad yields and the autophagy inhibitor 3-methyladenine (3-MA) reduced Ad replication (166). Our studies have also shown that autophagy may generate decomposed cellular molecules as nutrition to support Ad replication. Thus, an autophagy inducer may improve virus oncolytic therapy.

Autophagy is a process involving the lysosomal degradation and recycling of cellular proteins and cytoplasmic organelles (178). Environment stressors such as nutrient starvation and pathogen infection induce autophagy. Autophagy initiates from membrane structures called phagophores $(178,223,224)$, which engulf cellular and cytoplasmic components, followed by elongation and recruitment of microtubule-associated protein 1 light chanin 3 (LC3) to form the characteristic double-membrane autophagosome. Cytoplasmic form LC3-I and lipidated form LC3-II are two forms of LC3 post-transcriptionally produced in cells (225-227). LC3 is immediately processed into LC3-I after synthesis. During the autophagy process, LC3-I is cleaved by cysteine protease Atg4 to generate lipidated form LC3-II that localizes on autophagosome membranes $(224,228)$. The amount of LC3-II or the LC3-II/LC3-I ratio can be used to estimate the degree of autophagosome formation $(225,226,229)$. Autophagosomes eventually fuse into lysosomes to form autolysosomes, in which the inner components undergo the 
degradation process and produce amino acids and fatty acids for reuse in cells. Rapamycin, the inhibitor of the mammalian target of rapamycin (mTOR) (230, 231), has been shown to induce autophagy and inhibit proliferation of malignant glioma cells (232). Autophagy is negatively regulated by the PI3K-AKT-mTOR pathway. Via inhibiting the negative regulation of mTOR signaling, rapamycin indirectly enhances autophagy.

Using a tumor-specific promoter to regulate Ad E1A expression is a general effort to control selective replication of vectors in cancer cells and cause oncolysis. The proteins encoded by the E1a region, expressed immediately after infection, then modulate the cell cycle, recruit cellular proteins, and produce viral proteins to process viral DNA replication (37). However, all known tumor-specific promoters are relative weak compared with the native promoter of the Ad E1a gene (159, 161). In addition, Ad infection can cause strong repression of most cellular promoters, as indicated in our published microarray study (79). Vectors driven by tumor-specific promoters generally elicit low potency and do not work as efficiently as d/1520, which contains the native E1a promoter and is applied in current tumor treatments $(159,161)$. However, the native E1a promoter does not exhibit selectivity and therefore has side effects, such as virus replication in noncancerous cells $(155,156)$. Obviously, the selection of promoters in vector construction should consider the negative effects imposed by virus infection on those promoters. We thus have constructed a novel E1b-deleted oncolytic Ad-cycE, in which Ad E1a gene is driven by the cyclin E promoter. Cyclin E is 
known to regulate DNA replication and promote the S-phase entry $(110,111)$. Cyclin E overexpression is frequently detected in many types of cancers, including lung cancer (87). Recent studies also showed that overexpression of cyclin E can trigger lung cancers in transgenic mice $(86,115)$. Our previous studies revealed that the replication of E1b55K-deleted Ads is significantly repressed in $\mathrm{G}_{0}$-arrested normal cells $(56,123)$, in which the cyclin $\mathrm{E}$ promoter is restricted. We have also demonstrated that the activity of cyclin E promoter in cancer cells is further augmented after Ad infection $(56,165)$. As the replication of E1b-deleted Ad-cycE depends on the activation of cyclin E promoter, Ad-cycE replication may be enhanced in cancer cells and repressed in normal cells.

In this study, we applied novel tumor-specific Ad-cycE and rapamycin in combination to enhance oncolytic effects. We show that Ad-cycE is competent to replicate in human lung cancer cells but not in the normal lung cells and that the combination of oncolytic Ad-cycE and the autophagy inducer rapamycin elicits synergistic inhibition effects. We also reveal that rapamycin increases Ad E1A expression and virus production. Our studies have clearly shown that autophagy inducers as chemotherapeutic agents are capable of increasing adenoviral replication and oncolysis. Thus the combination of autophagy-associated chemotherapy and oncolytic virotherapy may be a new approach to improve future cancer treatment. 


\subsection{Materials and Methods}

Cell lines and culture conditions. HEK 293 (ATCC no. CRL-1573), WI-38 human lung fibroblast (ATCC no. CCL-75), MCF10A human mammary epithelial (ATCC no. CRL-10317), MDA-MB-231 human breast cancer (ATCC no. HTB-26), A549 (ATCC no. CCL-185) and H1299 (ATCC no. CRL-5803) human lung cancer cell lines were purchased from the American Type Culture Collection (Rockville, MD). WI-38 human lung fibroblast cell line has the properties of primary cells with a finite lifetime of 50 population doublings (174). MCF10A human mammary epithelial cell line is an immortalized but non-transformed human breast epithelial cell line $(233,234)$. WI-38 cells were cultured in minimal essential medium (MEM) Alpha GlutaMAX with $0.1 \mathrm{mM}$ non-essential amino acids and $1.0 \mathrm{mM}$ sodium pyruvate. MCF10A cells were cultured in DMEM/F12K with $20 \mathrm{ng} / \mathrm{ml}$ EGF, $0.5 \mu \mathrm{g} / \mathrm{ml}$ Hydrocortisone, and $10 \mu \mathrm{g} / \mathrm{ml}$ insulin. HEK 293, A549 and MDA-MB-231 cells were cultured in DMEM. All media were supplemented with 5\% (for MCF10A cells) or $10 \%$ (for the other cells) fetal bovine serum (FBS) and penicillin/streptomycin $(100 \mathrm{U} / \mathrm{ml})$. Cells were cultured in a $5 \% \mathrm{CO}_{2}$ incubator at $37^{\circ} \mathrm{C}$. All cell culture reagents were obtained from Gibco BRL (Bethesda, MD).

Adenoviral vectors. Figure 5.1 depicts the structures of the adenoviruses applied in this study. Wild-type adenovirus type 5 (Adwt, ATCC no. VR-5) was used as a replication-competent control. AdCMV/GFP, an Ad vector with E1 deletion carrying a green fluorescent protein (GFP), was used as a replication-defective control (164). Ad-cycE is a novel E1b-deleted oncolytic 
vector carrying a human cyclin $\mathrm{E}$ promoter driving an intact $\mathrm{E} 1 \mathrm{~A}$ expression cassette. The endogenous E1a promoter was deleted and a human cyclin E promoter (GenBank ID: X95406 (168)) was inserted to replace the deleted E1a promoter in Ad-cycE. Therefore, Ad-cycE contains a human cyclin E promoter to control E1a open reading frames (ORF). The details of Ad-cycE construction will be reported separately in our preparing report. All of the vectors created and used in this study are based on the backbone of wild-type Ad type 5 . 


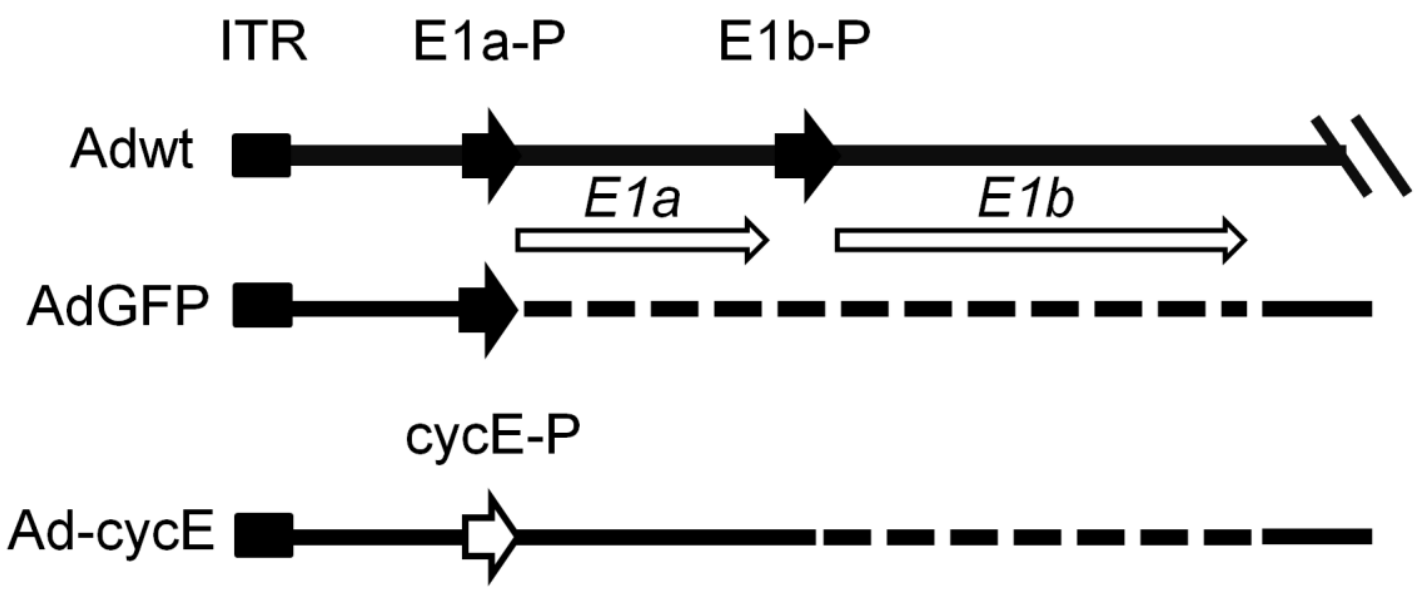

Figure 5.1. Structure of the Ads. The wild-type Ad (Adwt) with the E1a and E1b genes and their endogenous promoters is shown at the top. The left inverted terminal repeat (ITR), the promoters for E1a gene and E1b genes (E1a-P and $E 1 b-P)$ and the E1a and E1b open reading frames are indicated. The solid lines represent Adwt regions in these viruses, and the dashed lines represent the deleted regions. AdGFP contains the complete deletion of $E 1 a$ and $E 1 b$ regions and their promoters. Ad-cycE contains the deletion of $E 1 b$ region and a cyclin $\mathrm{E}$ promoter (cycE-P) was inserted to replace the deleted E1a promoter. 
Cytotoxicity assay. Cells were seeded into 24-well plates at a density of $2.5 \times 10^{4}$ (cells/well) and cultured under the indicated conditions. After 72 hours, Cytotoxicity was assessed with crystal violet staining (170). Cells were fixed and stained with $1 \%$ crystal violet followed by washing with water to remove excess dye. The dye was solubilized with $2 \%$ SDS and the absorbance of the solubilized stain was measured at $590 \mathrm{~nm}$ using a Synergy HT Multi-Mode Microplate Reader (Bio-Tek, Winooski, VT). The OD values were quantitated into the cell viability \% by the formula, cell viability $\%=(O D$ value of experimental group / OD value of control group) x 100\%. Rapamycin and viruses were diluted with corresponding culture media. The $0 \mathrm{nM}$ control group was treated with the diluents and was calculated as $100 \%$ of cell viability in the assay (171).

Analyses of combination effects of rapamycin and Ad-cycE. In this study, an additive effect refers to a combined effect of drugs that produces the sum of their individual effects; synergism is the combined effect of drugs which is greater than the sum of individual effects, and antagonism is the combined effect of drugs which is less than the sum of individual effects $(235,236)$. The combined effects of rapamycin and Ad-cycE on cell viability were analyzed with the median-effect methods of Chou and Talalay (237) using CalcuSyn software (Biosoft, Ferguson, $\mathrm{MO})$. The combination index $(\mathrm{Cl})$ values were used to evaluate the interaction between the drug and virus. For the fraction of virus affected combination index (Fa-Cl) plot analysis, a $\mathrm{Cl}<1$ is defined as synergism, a $\mathrm{Cl}=1$ is defined as an additive effect, and a $\mathrm{Cl}>1$ is defined as antagonism. The data were confirmed 
with the isobologram method $(238,239)$. The diagonal curves connecting the $x$ and $y$-axes were calculated from single treatments to represent the additive effect for the theoretical combinations of two treatments at the specific effective doses. If the data points fall on the lower left of the diagonal, the combination is regarded as synergism. If the experimental data points of the drug combination fall on the diagonal, the combination is regarded as an additive effect. If the data points fall on the upper right of the diagonal, the combination is regarded as antagonism.

Viral titration. Cells were seeded into 6 -well plates at a density of $2 \times 10^{5}$ (cells/well) and treated under the indicated conditions. Total infected cells and culture supernatants were collected at $48 \mathrm{hr}$ postinfection (p.i.) and lysed to release virus particles with three cycles of freezing and thawing. The viral yields were determined by the infective unit method as described previously $(120,121)$. HEK 293 cells were seeded in 96-well plates at a density of $10^{3}$ (cells/well) and then infected with 10-fold serially diluted viruses. CPE was recorded and scored after incubation for 7 days.

Western blot analysis. Cells were harvested and lysed with CDK2 lysis buffer (20 mM Tris pH 7.5, $150 \mathrm{mM} \mathrm{NaCl}, 5 \mathrm{mM} \mathrm{MgCl} 2,0.5 \%$ Nonidet P-40, 0.1\% Brij 35, $5 \mathrm{mM}$ sodium glycerophosphate, $1 \mathrm{mM}$ sodium vanadate, $1 \mathrm{mM}$ dithiothreitol). The Western blot analyses were performed as described previously (123). $25 \mu \mathrm{g}$ of cell lysates were electrophoresed through 10 or $12 \%$ SDS-polyacrylamide gels and transferred onto an Immobilon-P Membrane (Millipore, Billerica, MA). The 
primary antibodies used in this study were rabbit anti-LC3 and actin (Sigma, St. Louis, MO), mouse anti-adenovirus type 5 E1A (BD Pharmingen, San Jose, CA), and rabbit anti-adenovirus type 5 antibody (Abcam, Cambridge, MA). Actin was used as an internal control. The membranes were then incubated with anti-mouse immunoglobulin $G$ ( $\lg G$ ) or anti-rabbit IgG peroxidase-linked species-specific whole antibody (GE Healthcare, Piscataway, NJ). Chemiluminescent detection was performed with ECL reagents according to the supplier's recommendations (GE Healthcare). The scanned band intensity was quantitated by Gel-pro Analyzer 4.0 software (Media Cybernetics, Bethesda, MD) according to the manufacturer's tutorial. Densitometric value for each band was expressed as integrated optical density (I.O.D.) and normalized with actin. The results were reported as the ratios of normalized band intensities of LC3-II to LC3-I.

Statistical analyses. All above experiments, except specifically indicated, were repeated at least three times. Quantitation results were reported as means \pm standard deviation (S.D.). The Pearson correlation coefficient $(r)$ was used to evaluate the correlations between the rapamycin concentrations and cell viability percentages by SAS software, Version 9.3 (SAS Institute Inc., Cary, NC) (240, 241). Statistical difference of the combination experiment was assessed with a Student's $t$-test. Statistical significance of difference was set at $p<0.05$. 


\subsection{Results}

Selective replication of Ad-cycE in cancer cells. Figure 5.1 depicts the structures of the adenoviruses applied in this study. Adwt was used as a replication-competent control. AdCMV/GFP, a vector with E1a and E1b deletion carrying a green fluorescent protein (GFP), was used as a replication-defective control. Our previous reports indicated that the cyclin E promoter is more active in lung cancer cells than in normal lung cells and oncolytic E1b-deleted Ad infection further elevates the promoter activation $(56,165)$. Thus, we replaced the native E1a promoter with the cyclin $\mathrm{E}$ promoter to generate Ad-cycE, a novel E1b-deleted oncolytic vector.

To determine the selectivity of Ad-cycE for cancer cells, we first examined the cytotoxicity of Ad-cycE on normal and cancer cell lines. WI-38 human lung fibroblast, MCF10A mammary epithelial, A549 and H1299 lung cancer and MDA-MB-231 breast cancer cells were infected with AdGFP, Adwt or Ad-cycE at 5 MOI. Ad-cycE replicated in A549 and H1299 human lung cancer, and MDA-MB-231 breast cancer cells and caused cytopathic effect (CPE) similar to that of Adwt (Fig. 5.2A, comparing panel hr and $\mathrm{i}, \mathrm{k}$ and I, $\mathrm{n}$ and o). The CPE became visible at $48 \mathrm{hr}$ and $72 \mathrm{hr}$ p.i., showing that the infected cells became circular and detached from the cell monolayer. Unlike Adwt, which indistinguishably induced CPE in noncancerous WI-38 human lung fibroblast and MCF10A mammary epithelial cells, Ad-cycE selectively induced CPE in all tested cancer cells (Fig. 5.2A, comparing panel b and c, e and f). 
WI-38, A549 and H1299 cells were infected with Adwt or Ad-cycE at $5 \mathrm{MOI}$ and the total infected cells and culture supernatants were collected at $72 \mathrm{hr}$ to examine the production of infectious virus particles. Figure 5.2B revealed that in noncancerous WI-38 human lung fibroblast cells, the virus yield of Adwt was significantly higher than that of $A d-c y c E(P=0.04)$; in $\mathrm{H} 1299$ human lung cancer cells, there was no significant difference between the virus yield of Adwt and Ad-cycE $(P=0.2)$; in $A 549$ human lung cancer cells, Adwt $\left(3.7 \times 10^{9}\right)$ and Ad-cycE $\left(8.3 \times 10^{8}\right)$ achieved a high level of virus yield, indicating the replication property of $\mathrm{Ad}$-cycE in lung cancer cells. The aggregated data from this experiment shows that Ad-cycE can selectively replicate in and efficiently destroy cancer cells but poorly replicates in noncancerous cells.

Previous studies indicate that replication-competent oncolytic Ads could produce the essential Ad E1 proteins to support the replication of replication-defective E1-deleted Ads co-transduced in vitro or in vivo $(165,242,243)$. To further verify selective replication capability of Ad-cycE in cancer cells, A549 human lung cancer cell line with constitutive cyclin E production (56) was chosen for the following experiment. A549 cells were infected with 5MOI AdGFP alone, or AdGFP plus an additional Ad (AdGFP+AdlacZ, AdGFP+Adwt or AdGFP+Ad-cycE). Figure 5.2C showed that the non-replicative AdGFP maintained the original level of infectivity at day 1 and day 2 (compare panels a and b). Also, with coinfection of AdGFP and non-replicative AdlacZ, the fluorescent cell numbers did not change (compare panels $c$ and d). Yet with the addition of Adwt (compare panels e and f) or Ad-cycE (compare panels $g$ and $h$ ), 
we detected an increase of fluorescent cell numbers from day 1 to day 2 , suggesting that efficacy of Ad-cycE replication in cancer cells is comparable with the wild-type Ad. 
A.

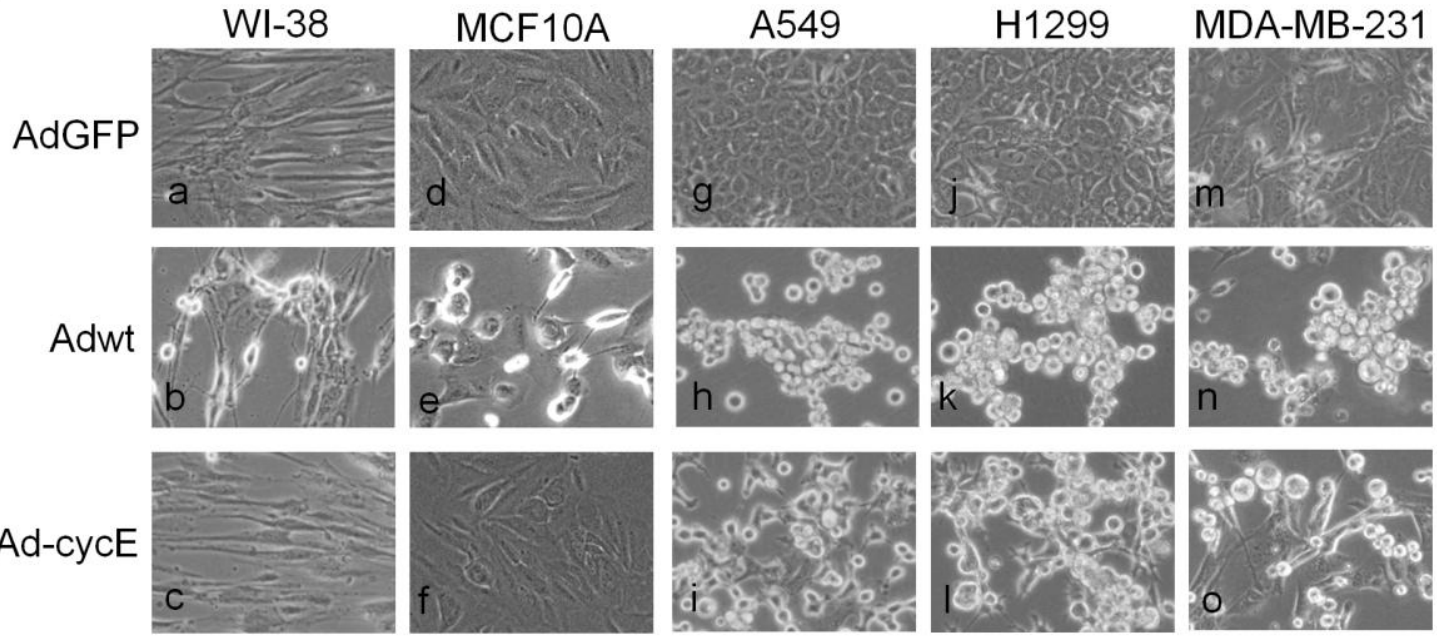

B.
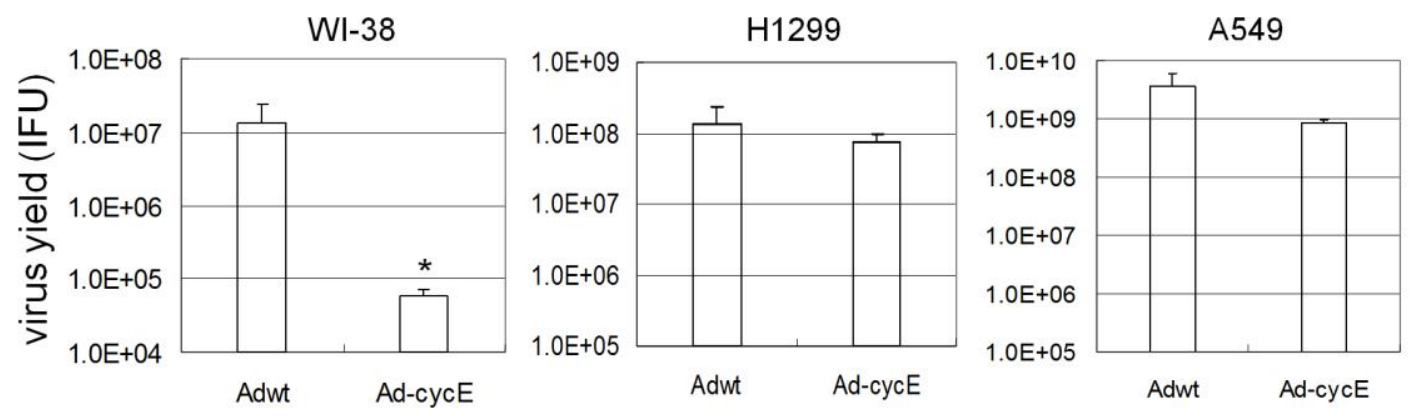

C. AdGFP only AdGFP+AdlacZ AdGFP+Adwt AdGFP+Ad-cycE

day 1
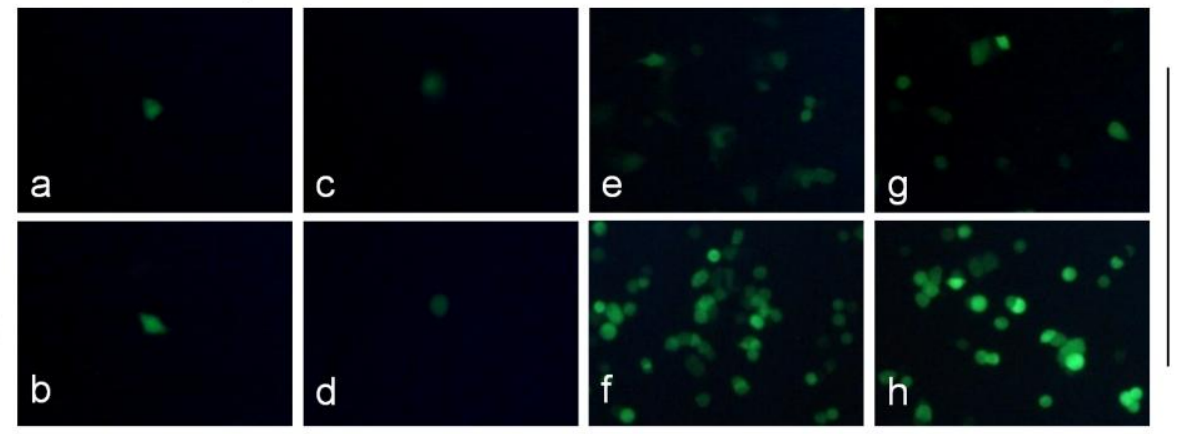

A549

day 2
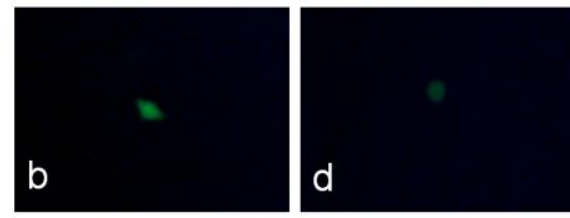

A549 
Figure 5.2. Selective oncolytic replication of Ad-cycE. (A) WI-38, MCF10A, A549, H1299 and MDA-MB-231 cells were infected with AdGFP, Adwt, or Ad-cycE at $5 \mathrm{MOI}$. CPE was observed at $48 \mathrm{hr}$ or $72 \mathrm{hr}$ p.i and photographed with an inverted microscope Olympus CKX41. (B) Viral yields produced in WI-38, H1299 and A549 cells were determined at $72 \mathrm{hr}$ p.i. with the infection unit method. The values represent the means \pm S.D. of independent triplicate. ${ }^{*} P<0.05$, Student's t-test. (C) A549 cells were co-infected with $5 \mathrm{MOI}$ AdGFP and mock-infection, AdlacZ, Adwt or Ad-cycE. All fluorescent microscopy is taken at day 1 and day 2 p.i. with an Olympus IX50 microscope (original magnification of X100). 
Rapamycin induces autophagy and inhibits lung cancer cell growth. Rapamycin has been shown to induce autophagy and inhibit proliferation of malignant glioma cells (232). We investigated whether rapamycin can induce autophagy in A549 lung cancer cells. The cells were treated with $0 \mathrm{nM}, 100 \mathrm{nM}$, and $200 \mathrm{nM}$ rapamycin for $24 \mathrm{hr}$. Western blot was used to determine the conversion of LC3-I to LC3-II, which is one of the representative characteristics of autophagy activation. LC3 is immediately processed into LC3-I after synthesis. Then the cytoplasmic form LC3-I is cleaved by cysteine protease Atg4 to generate lipidated form LC3-II that specifically localizes to autophagosome membranes (228). Thus the amount of LC3-II or the LC3-II/LC3-I ratio can be applied to estimate the abundance of autophagosomes $(225,226,229)$. Figure $5.3 \mathrm{~A}$ shows two forms of LC3, the upper band corresponding to LC3-I and lower band corresponding to LC3-II (225). Compared with the $0 \mathrm{nM}$-control group (the ratio of LC3-II/LC3-I = 0.82), the $100 \mathrm{nM}$ and $200 \mathrm{nM}$ rapamycin treatments increased the amount of LC3-II and caused the ratio of LC3-II/LC3-I to 2.75 and 2.88, respectively, indicating the induction of autophagy. Next we examined the effect of rapamycin on A549 cell growth. The cells were treated with $0 \mathrm{nM}, 100 \mathrm{nM}, 200 \mathrm{nM}$, $400 \mathrm{nM}, 600 \mathrm{nM}$ and $800 \mathrm{nM}$ rapamycin for $72 \mathrm{hr}$. The cell viability was determined with crystal violet staining and quantitated into cell viability percentages. The results showed that rapamycin decreased cell viability in a dose-dependent manner at $72 \mathrm{hr}$ compared to the $0 \mathrm{nM}$-control group ( $r=$ $-0.69033, P=0.0002)$ (Fig. 5.3B). 
A.

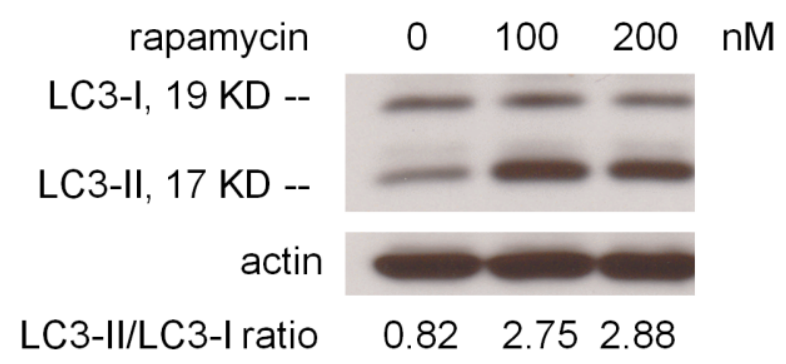

B.

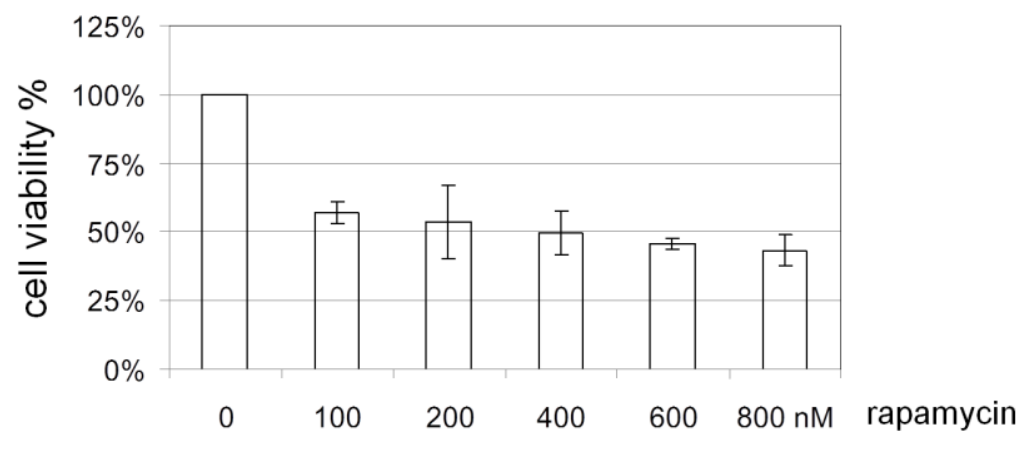

Figure 5.3. Effects of rapamycin on cytotoxicity and autophagy. A549 cells were treated with $0 \mathrm{nM}, 100 \mathrm{nM}$, and $200 \mathrm{nM}$ ramamycin (LC Laboratories, Woburn, MA) and collected at $24 \mathrm{hr}$ after treatment. (A) Cell lysates were immunoblotted for LC3 and actin. Actin was used as a loading control. The values indicate the ratios of normalized band intensities of LC3-II to LC3-I. (B) A549 cells were treated with $0 \mathrm{nM}, 100 \mathrm{nM}, 200 \mathrm{nM}, 400 \mathrm{nM}, 600 \mathrm{nM}$ and $800 \mathrm{nM}$ ramamycin. The cell viability \% was determined at $72 \mathrm{hr}$ after treatment. The values of cell viability $\%$ represent the means \pm S.D. of independent quadruplicate compared with the $0 \mathrm{nM}$-control group. 


\section{Combination of rapamycin and Ad-cycE elicits stronger cytotoxicity than}

single treatment alone. We first chose $200 \mathrm{nM}$ rapamycin as the working condition and tested the combination effects of rapamycin with different MOls of Ad-cycE on lung cancer cell growth. Figure 5.4A shows a difference between the cell viability percentage of treatment with $A d-c y c E$ alone and Ad-cycE in combination with rapamycin. Our results show that Ad-cycE in combination with rapamycin induces greater CPE in A549 lung cancer cells than either treatment alone. The distinction can be clearly seen in both $0.5 \mathrm{MOI} A d-c y c E$ and $1 \mathrm{MOI}$ Ad-cycE. Statistical Student's $t$-tests confirmed the significant difference. Treatments with $200 \mathrm{nM}$ rapamycin or $1 \mathrm{MOI}$ Ad-cycE both resulted in the cell viability of about $50 \%$ (53.9\% for rapamycin and $52 \%$ for Ad-cycE). Combination of Ad-cycE and rapamycin decreased cell viability to $23.6 \%(P=0.00000011)$. We repeated the experiment with even lower dose of $A d-c y c E(0.5 \mathrm{MOI})$, which only is able to induce slight CPE. $0.5 \mathrm{MOI}$ Ad-cycE only caused $73.9 \%$ of the cell viability $(P=0.0052)$, but combination with $200 \mathrm{nM}$ rapamycin caused $39.4 \%$ of the cell viability $(P=0.0000000002)$. The cell morphology was photographed with an inverted microscope on day 2 (Fig 5.4B). These results suggest that rapamycin in combination with Ad-cycE elicits greater cytotoxicity on A549 cells even with a low MOI of Ad-cycE. 
A.

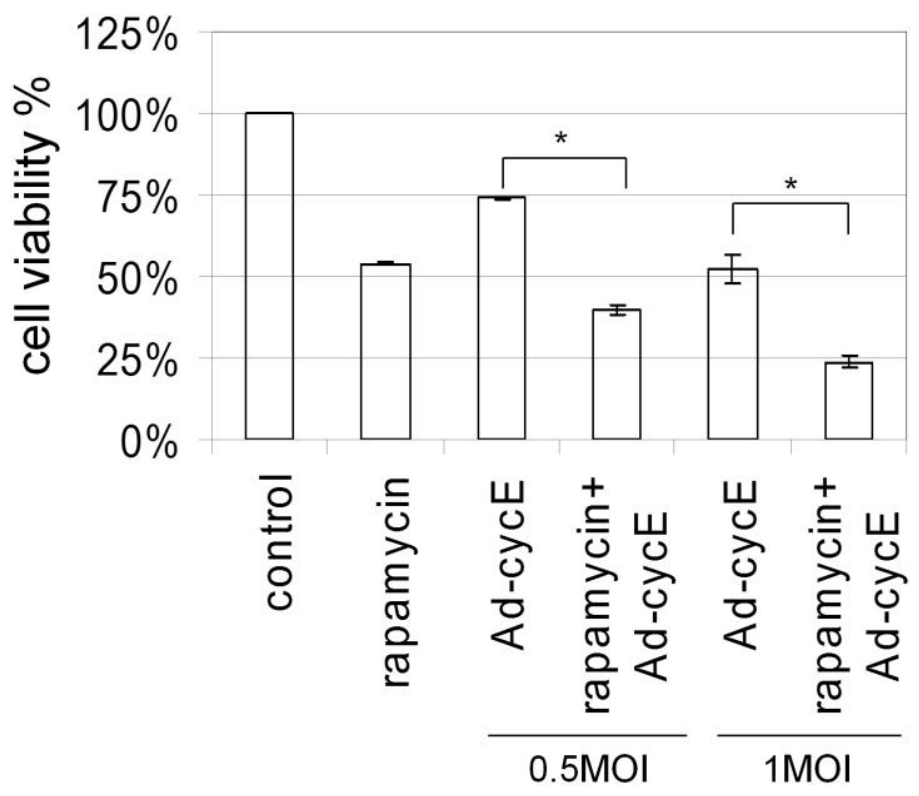

B.

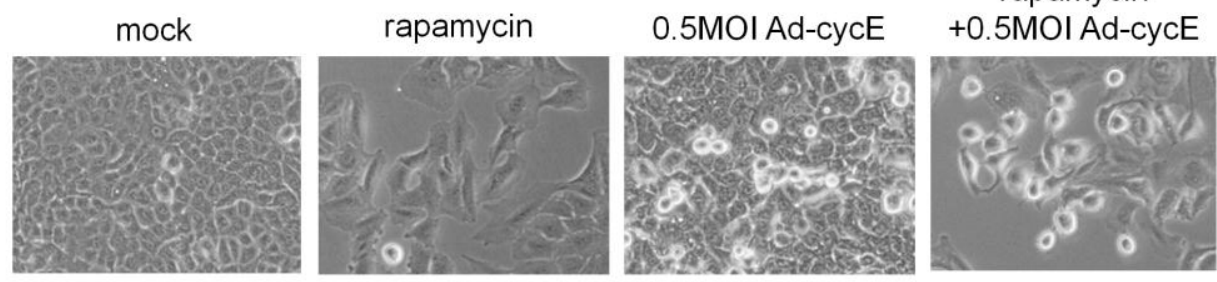

Figure 5.4. Effects of combination of rapamycin and Ad-cycE on A549 cells. A549 cells were non-treated or treated with $200 \mathrm{nM}$ rapamycin alone, 0.5 or 1 $\mathrm{MOI}$ Ad-cycE alone, or the combination treatment of $200 \mathrm{nM}$ rapamycin and 0.5 or $1 \mathrm{MOI}$ Ad-cycE. (A) The results were quantitated into cell viability \%. The values of cell viability $\%$ represent the means \pm S.D. of independent triplicate compared with the mock-control group. * $\mathrm{P}<0.05$, Student's $t$-test. (B) CPE was photographed at a magnification of X100 at $48 \mathrm{hr}$ p.i. 
Rapamycin increases Ad E1A expression and oncolytic replication. The stronger antitumor effect in the combination treatment may be generated from the sum of the effect of two individual treatments or even a synergistic effect (one treatment may increase the efficacy of the other). To understand the mechanism by which rapamycin in combination with Ad-cycE caused stronger antitumor effects, we first examined the production of virus particles, comparing virus alone with the combination groups. Rapamycin treatment led to a 4.25 -fold increase in virus yield compared to the virus alone group (Fig. 5.5A). This suggests that rapamycin increases the production of Ad-cycE in A549 cells, resulting in stronger antitumor effects than either drug or virus alone. Next we examined the E1A expression to determine the mechanism by which rapamycin may contribute to the increased production of $\mathrm{Ad}$ vectors. E1A is the crucial protein which is expressed immediately after infection and initiates the virus replication cycle (37). Ad E1A protein expression was examined at 18 hours and identified as multiple bands at $35-46 \mathrm{kDa}$ generated from the alternative splicing of $\mathrm{E} 1 \mathrm{~A}$ transcripts (196). As shown in Figure 5.5B, rapamycin stimulates elevated E1 A expression in the combination group when compared to Ad-cycE alone. Taken together, the results suggest that rapamycin increases oncolytic replication of Ad-cycE in A549 cells and enhances E1A expression. 
A.

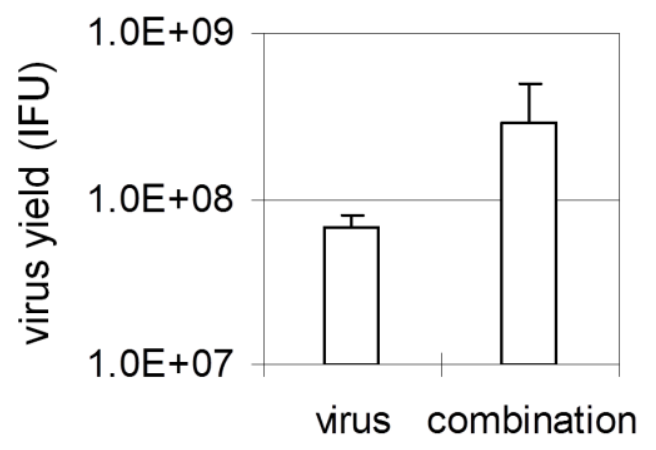

B.

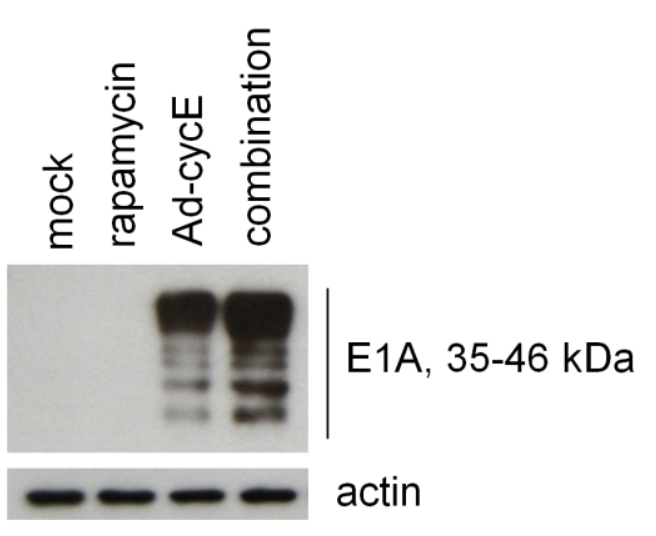

Figure 5.5. Effects of rapamycin on the oncolytic replication of Ad-cycE. A549 cells were non-treated or treated with $200 \mathrm{nM}$ rapamycin alone, $0.5 \mathrm{MOI}$ Ad-cycE alone, combination treatment of $200 \mathrm{nM}$ rapamycin and $0.5 \mathrm{MOI}$ Ad-cycE. (A) Viral yields were determined at $48 \mathrm{hr}$ p.i. with the infection unit method. The values represent the means \pm S.D. of independent triplicate. (B) Cell lysates harvested at $18 \mathrm{hr}$ p.i. were immunoblotted for Ad E1A and actin. Actin was used as a loading control. 


\section{Combination of rapamycin and Ad-cycE elicits synergistic antitumor effects.}

To determine whether combination of rapamycin and Ad-cycE produce synergistic antitumor effects, we evaluated the combination treatments with Calcusyn (Biosoft, Ferguson, MO). The combination experiment was performed by adopting the constant ratio drug combination design proposed by Chou and Talalay (237). A549 human lung cancer cells were treated with rapamycin alone (from $100 \mathrm{nM}$ to $700 \mathrm{nM}$ ), Ad-cycE alone (from $0.5 \mathrm{MOI}$ to $3.5 \mathrm{MOI}$ ) or a combination of rapamycin (nM) with Ad-cycE (MOI) at the constant ratio of 200:1 for $96 \mathrm{hr}$. Concordant with the results shown in Figure 5.4, combination of rapamycin and Ad-cycE caused a greater cytotoxicity than either treatment alone (Fig. 5.6A). We then evaluated these quantitated data by fraction affected versus combination index ( $\mathrm{Fa}-\mathrm{Cl})$ with CalcuSyn software (Biosoft, Ferguson, MO) (Fig. 5.6B). The X-marks represent the combination index $(\mathrm{Cl})$ values of the combination treatment groups. The $\mathrm{Cl}$ values are $0.326,0.512,0.506,0.642$, and 0.689 for $100 \mathrm{nM}$ rapamycin plus 0.5 MOI Ad-cycE, $200 \mathrm{nM}$ rapamycin plus $1 \mathrm{MOI} A d-c y c E, 300 \mathrm{nM}$ rapamycin plus 1.5 MOI Ad-cycE, $600 \mathrm{nM}$ rapamycin plus $3 \mathrm{MOI}$ Ad-cycE, and $700 \mathrm{nM}$ rapamycin

plus 3.5 MOI Ad-cycE, respectively. The middle curve line represents the simulated combination index values of the combination treatment groups surrounded by two lines of algebraic estimations of the $95 \%$ confidence intervals. All experimental $\mathrm{Cl}$ values at the tested ratio were significantly $<1$ and between the two confidence lines, indicating synergism of combination treatments.

Since rapamycin and Ad treatments have entirely independent modes of action, 
the conservative isobologram method $(237,244)$ was also applied here to confirm the above $\mathrm{Fa}-\mathrm{Cl}$ results. The effective concentration $\mathrm{EC}_{50}, \mathrm{EC}_{75}$ and $\mathrm{EC}_{90}$ refer to the concentration of a drug or the combination of the two drugs that induces $50 \%$, $75 \%$ and $90 \%$ inhibition of cell viability (236). Figure $5.6 \mathrm{C}$ shows the conservative isobologram plots of $\mathrm{EC}_{50}, \mathrm{EC}_{75}$ and $\mathrm{EC}_{90}$, separately. In the conservative isobologram plot, the curve connecting each axis indicates the simulated additive effect for $\mathrm{EC}_{50}, \mathrm{EC}_{75}$ and $\mathrm{EC}_{90}$, respectively. The experimental $\mathrm{EC}_{50}, \mathrm{EC}_{75}$ and $\mathrm{EC}_{90}$ doses of the combination treatment groups are displayed as the single point indicated by the arrow. The point values of the $\mathrm{EC}_{50}, \mathrm{EC}_{75}$ and $\mathrm{EC}_{90}$ for the combination treatments all fall below their diagonal lines for simulated additive effects, indicating that significantly lower doses of rapamycin and Ad-cycE are therapeutically effective when combined. For example, in $\mathrm{EC}_{50}$ isobologram, from the simulated curve of the additive effect it shows that to reach $50 \%$ inhibition of cell viability requires at least $800 \mathrm{nM}$ rapamycin or $1 \mathrm{MOI}$ Ad-cycE. However, with the combination of rapamycin and Ad-cycE it takes a relatively low dose (50 nM rapamycin plus $0.25 \mathrm{MOI} A d-c y c E)$ to achieve the same efficacy, suggesting combination treatment elicits a greater effect (synergism) than an additive effect. These results in Figure 5.6A, B and $\mathrm{C}$ have all demonstrated that the combination of rapamycin with Ad-cycE elicits a synergistic antitumor effect in A549 human lung cancer cells at the tested concentration ratio. 
A.

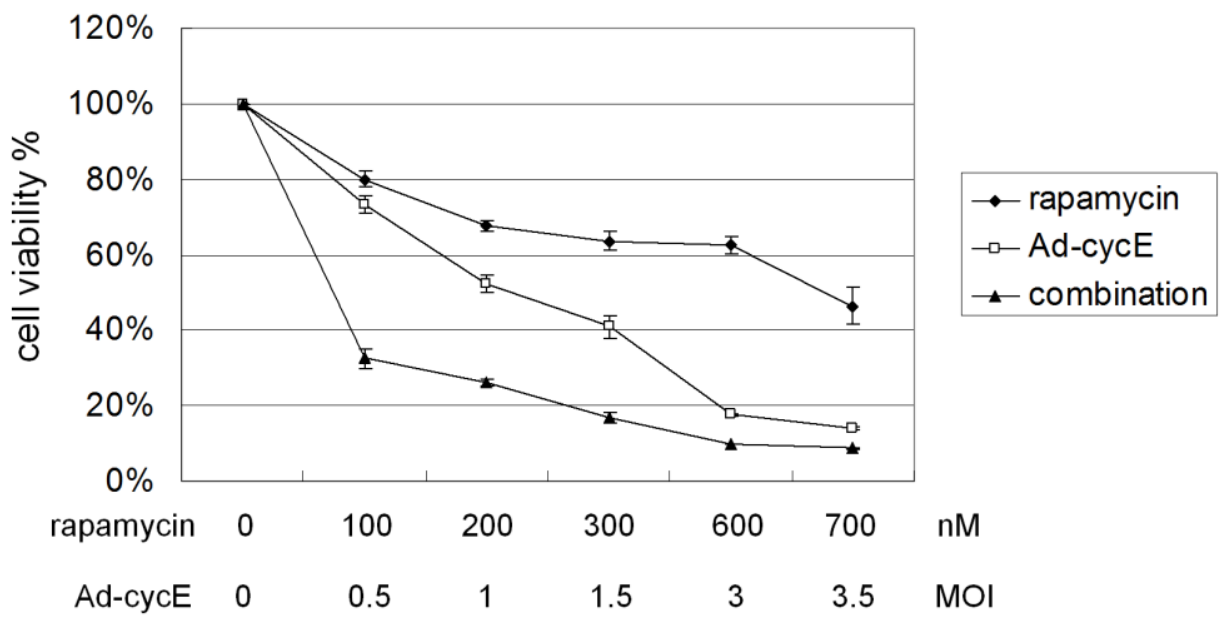

B.

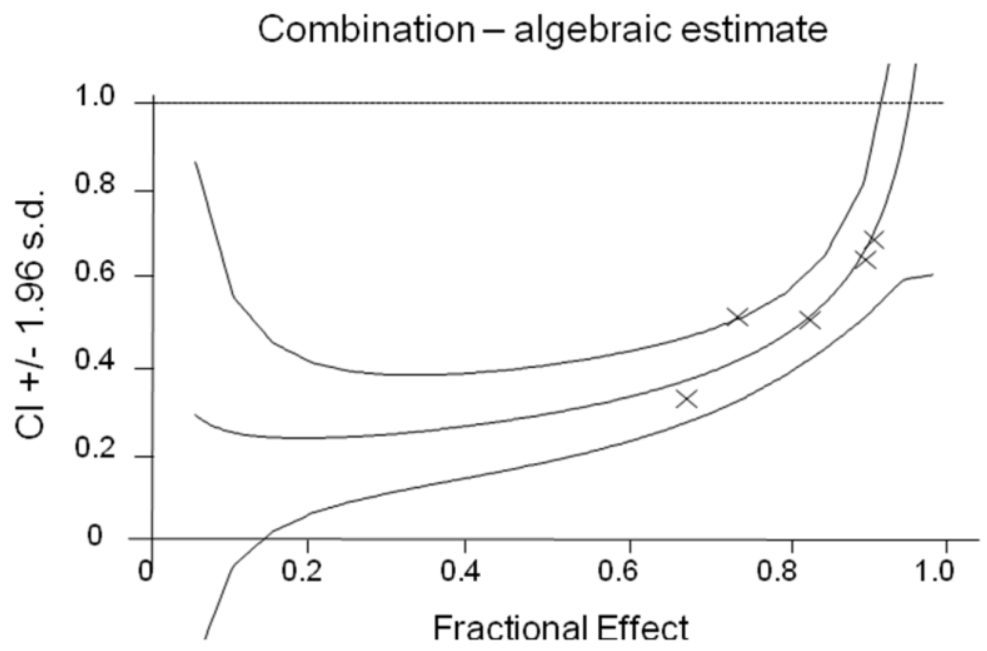

C.

Conservative Isobologram
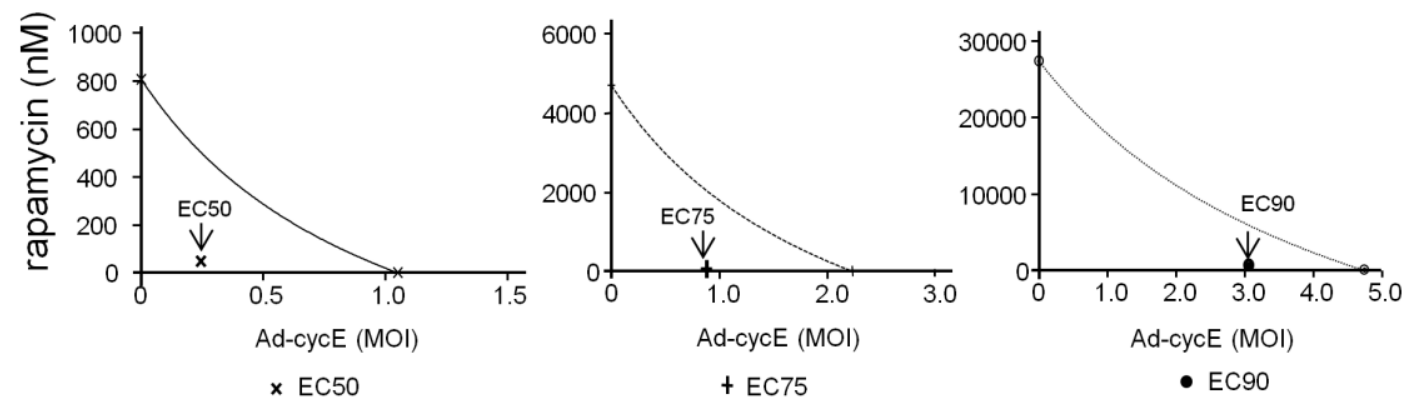
Figure 5.6. Analysis by Calcusyn (Biosoft, Ferguson, MO) of the interaction between rapamycin and Ad-cycE on A549 cells. Cells were treated with rapamycin alone, Ad-cycE alone or combination of both for $96 \mathrm{hr}$. (A) The results were quantitated into cell viability $\%$. The values of cell viability $\%$ represent the means \pm S.D. of independent triplicate compared with the mock-control group. (B) The quantitated cell viability data were analyzed by CalcuSyn software. The X-marks represent the combination index $(\mathrm{Cl})$ values of the combination treatment groups. The middle curve line represents the simulated combination index values of the combination treatment groups surrounded by two lines of algebraic estimations of the $95 \%$ confidence intervals. (C) The effective concentration $\mathrm{EC}_{50}, \mathrm{EC}_{75}$ and $\mathrm{EC}_{90}$ refer to the concentration of a drug or combination of drugs that induces $50 \%, 75 \%$ and $90 \%$ inhibition of cell viability. In the conservative isobologram plot, the three curves of the expected $\mathrm{EC}_{50}, \mathrm{EC}_{75}$ and $\mathrm{EC}_{90}$ additive effect lines for the combination treatments are labeled; the individual points of $\mathrm{EC}_{50}, \mathrm{EC}_{75}$ and $\mathrm{EC}_{90}$ for the combination treatments were indicted by arrows and located below their additive interaction lines, respectively. 
In addition, we examined the combination effect of rapamycin and Ad-cycE on MDA-MB-231 human breast cancer cell line, which has been reported as a non-permissive cancer cell line for oncolytic Ads replication (56). As we observed in A549 cells, the combination of rapamycin and Ad-cycE induced a greater cytotoxicity than either treatment alone in MDA-MB-231 cells (Fig. 5.7A) and the therapeutic effect was significantly enhanced by the synergism of combination treatments (Fig. 5.7B). 
A.

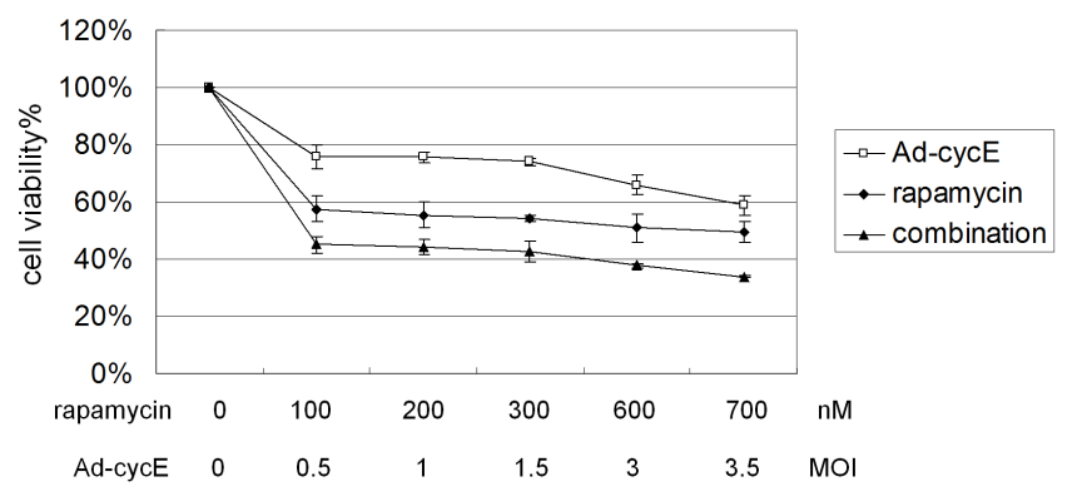

B.

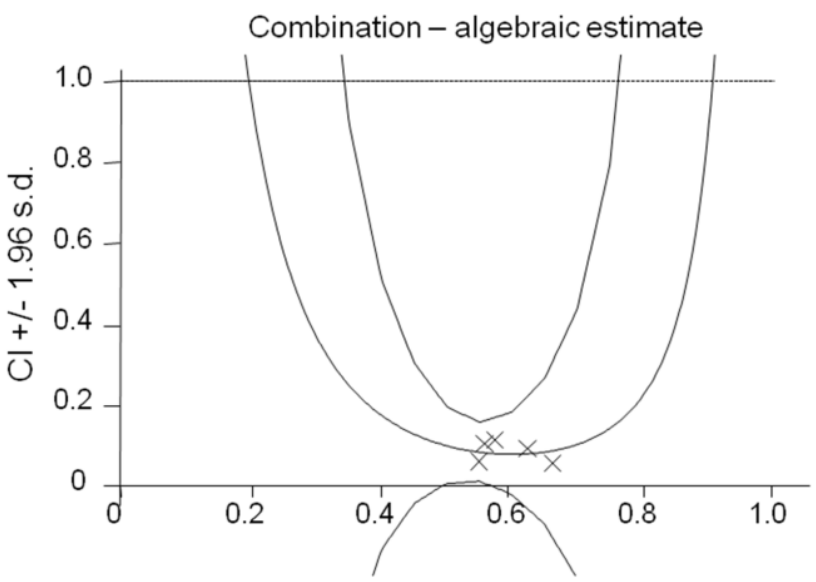

Fractional Effect

Figure 5.7. Effects of combination of rapamycin and Ad-cycE on MDA-MB-231 cells. (A) Cells were treated with rapamycin alone, Ad-cycE alone or combination of both for $96 \mathrm{hr}$. The results were quantitated into cell viability \%. The values of cell viability $\%$ represent the means \pm S.D. of independent triplicate compared with the mock-control group. (B) The quantitated cell viability data were analyzed by CalcuSyn software. In the fraction affected versus combination index (Fa-Cl) plot, all experimental $\mathrm{Cl}$ values at the tested ratio were significantly $<1$ and within the confidence lines. 
To determine whether the findings with rapamycin and oncolytic Ad-cycE may apply to wild-type Ad, we tested the same conditions with the combination of rapamycin and Adwt. Consistent to the results shown in Figure 5.6 and 5.7, combination of rapamycin and Adwt also caused a greater cytotoxicity than either treatment alone (Fig. 5.8A). Fa-Cl plot showed that all $\mathrm{Cl}$ values at the tested concentration ratio were significantly $<1$ and between the two confidence lines, suggesting the synergism of rapamycin and Adwt (Fig. 5.8B). Our data indicated that the synergism is not only observed in the combination of rapamycin and oncolytic Ad-cycE but also in that of rapamycin and Adwt, suggesting the potential of applying rapamycin to the strategy of combination treatment with the other oncolytic Ads. 
A.

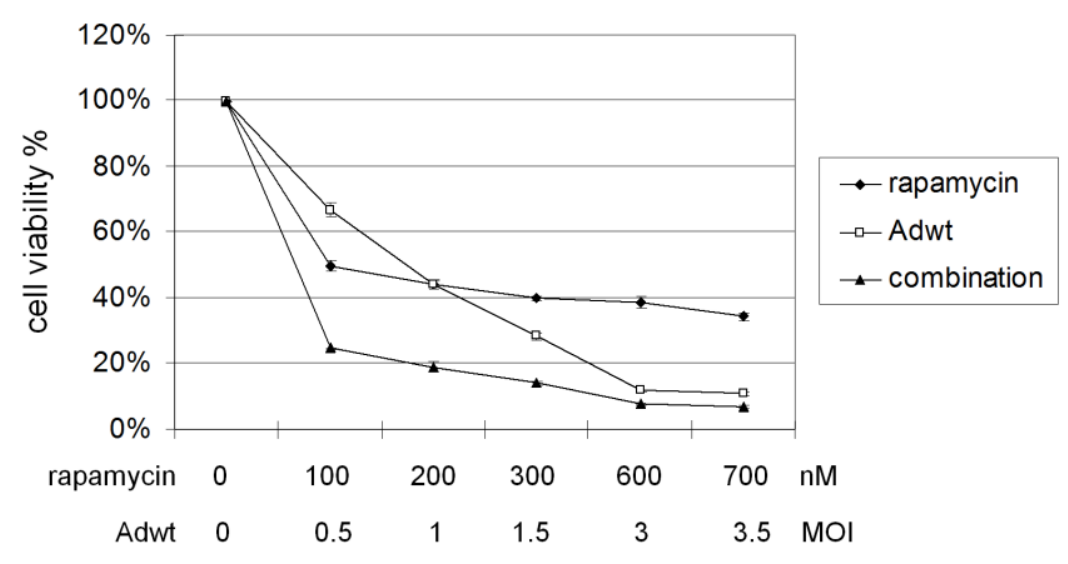

B.

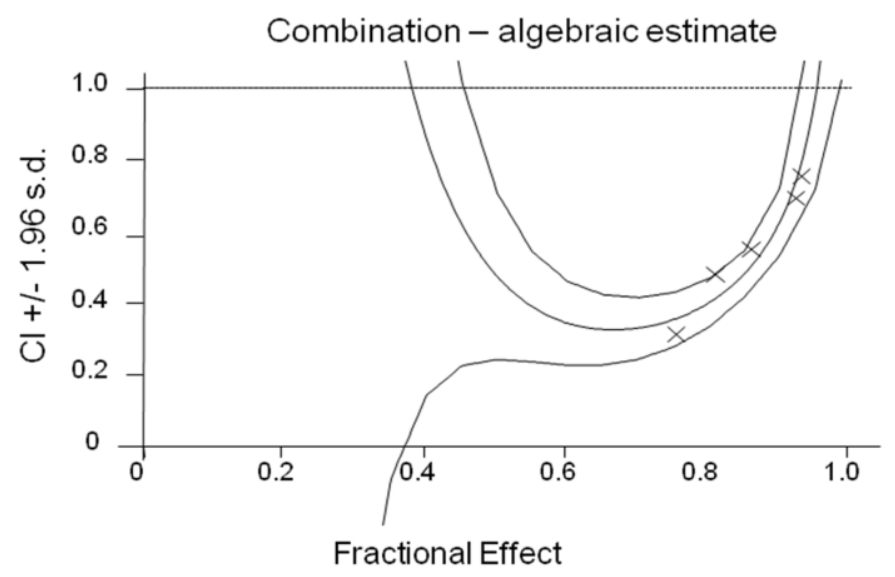

Figure 5.8. Effects of combination of rapamycin and wild-type Ad on A549 cells. (A) Cells were treated with rapamycin alone, Adwt alone or combination of both for $72 \mathrm{hr}$. The results were quantitated into cell viability \%. The values of cell viability $\%$ represent the means \pm S.D. of independent triplicate compared with the mock-control group. (B) The quantitated cell viability data were analyzed by CalcuSyn software. In the fraction affected versus combination index $(\mathrm{Fa}-\mathrm{Cl})$ plot, all experimental $\mathrm{Cl}$ values at the tested ratio were significantly $<1$ and within the confidence lines. 


\subsection{Discussion}

Oncolytic virotherapy has shown promising therapeutic results and is considered a potential approach for cancer therapy (245). The matchless advantage of this approach is that selective oncolytic effects are initiated by a small amount of viruses that spread to the surrounding regions until all cancer cells are destroyed (4). However, due to the current limitations, virus replication and diffusion are restricted in animal studies and clinical trials when the objectives carry tumors with large masses $(17,121,246)$. Viruses have difficulty penetrating massive tumors; this may be a reason for disappointing therapeutic outcomes. Developing new strategies to increase virus propagation in tumors is important in improving the efficiency of oncolytic virotherapy.

In our previous study we have shown that autophagy may generate decomposed cellular molecules as nutrients to support virus replication (166). Therefore we applied the autophagy inducer rapamycin to develop a combination strategy with oncolytic Ad-cycE. First, rapamycin-caused autophagy can generate more nutrients that can be used for building the viral particles $(166,247)$. Second, autophagy may increase virus particle release from dead cells that may benefit viral spread in tumors (248). Third, rapamycin has been applied to transplant recipients as an immunosuppressant to prevent organ rejection (249). The immunosuppressive properties of rapamycin mainly result from the inhibition of leukocyte activity and cytokine expression. Thus, rapamycin as an immunosuppressant may help virus to decrease host antiviral responses and 
improve virus distribution in tumors. Finally, autophagy-induced cell death has been applied as the new target in chemotherapy (250). Thus the antitumor effects can be enhanced by both rapamycin-caused autophagy and virus-mediated oncolysis.

We demonstrated that Ad-cycE selectively replicated in cancer cells. Ad-cycE in combination with autophagy-inducer rapamycin further induced synergistic antitumor effects. Rapamycin may also improve oncolytic therapy mediated by other viruses. Studies have shown that an autophagy mechanism is required for hepatitis B virus replication (251), the initiation of hepatitis C virus replication (252) and the promotion of viral replication of the RNA viruses such as poliovirus and rhinovirus (253). The new role of autophagy to help the virions of adenovirus type 2 (Ad2) to traffic in cells has also been discovered in a recent study (247). After the virus has been internalized into cells, high level of autophagosomes induced by autophagy are reported to fuse with the early endosomes containing virions and form amphisomes, creating an environment favoring the release of virions into cytosol. Here, we specifically observed that the autophagy inducer rapamycin increased the $\mathrm{E} 1 \mathrm{~A}$ expression and led to higher $\mathrm{Ad}-\mathrm{cyc} \mathrm{E}$ production. In agreement with our finding, Zeng and Carlin (2013) reported that starvation-induced autophagy enhanced the E1A expression and the viral progeny production of Ad2 in human airway epithelial cells (247). E1A is the crucial protein expressed immediately after infection and regulates the expression of multiple cellular and viral genes to initiate the virus replication cycle (37). 
Therefore, we reasoned that autophagy is not only able to generate nutrients for building viral particles, but is also able to increase the E1A expression of Ads, leading to higher virus production and the enhanced combination therapeutic effects.

mTOR pathway has been considered as a determinant regulator in the cellular metabolism (254). The mTOR inhibitor rapamycin has been reported to elicit diverse and paradoxical effects on the cellular metabolism. Some studies suggested that rapamycin decreases glucose metabolism (255-257) and mitochondrial oxidative functions in mammalian cells $(258,259)$, whereas some others suggested that rapamycin increases glycolysis and oxidative phosphorylation in the targeted cells $(260,261)$. Fang et al. (2013) pointed out that although detrimental metabolic changes were observed at early stages of rapamycin treatment in mice, the prolonged rapamycin treatment leaded to beneficial metabolic alterations, including increased insulin sensitivity, improved lipid profile and metabolism (262). Apparently, the discrepancy of those metabolic alternations by rapamycin likely depends on the natures of signaling pathways activated in the cell lines and the duration of treatment $(261,262)$. Under this circumstance, the relation between the metabolic alterations induced by mTOR inhibition and the adenoviral replication still remains unclear. Some DNA viruses such as adenovirus and human cytomegalovirus stimulate metabolic alternations such as glycolysis in the host cells to generate energy and essential elements for viral replication (263-265). Besides autophagy, the property of rapamycin to 
induce metabolic changes may be also utilized by adenovirus to create a beneficial environment for the viral replication.

Based on our previous work with the chemical CDK2 inhibitor roscovitine (164), we noticed that some chemotherapeutic agents with the kinase inhibition properties may inhibit oncolytic Ad replication and thus impair the outcome of oncolytic virotherapy in the combination therapy. It is important to select the chemotherapeutic agents without negative effects on oncolytic viruses when conducting the combination therapy. mTOR regulates several essential signal transduction pathways including the control of cell-cycle progression (254). As an mTOR inhibitor, one of the key functions of rapamycin is to inhibit cell-cycle progression (266). Rapamycin is reported to decrease cyclin D1 expression (267), reduce the kinase activity of cyclin D1/CDK4 and cyclin E/CDK2 complexes (268), and block the elimination of the CDK inhibitor p27 (269), leading to cell cycle arrest in $\mathrm{G}_{1}$-S-phase (266). The mechanism(s) by which oncolytic adenoviruses overcome the cell cycle arrest by rapamycin-induced mTOR inhibition requires the further study. Considering the possible negative effects of rapamycin on cell cyclins and cell-cycle progression, autophagy is likely to conduct a very important role for the rapamycin-enhanced virus replication in this study.

Our studies suggest a novel strategy involving targeting cyclin E overexpression in cancer cells and the properties of autophagy to enhance adenoviral oncolysis that could have a significant impact on clinical outcomes in cancer therapy. The 
combination of Ad-cycE and rapamycin can be further tested in vivo to evaluate the efficacy and efficiency for the clinical setting. Our findings also provide important information for future adenoviral vector development and the combination study for improving oncolytic virotherapy. 


\section{CHAPTER VI}

\section{SUMMARY AND FUTURE PERSPECTIVES}

Although E1b55K-deleted oncolytic Ads has been applied in many clinical trials and marketed for cancer therapies in China, the mechanism enabling tumor selectivity of the viruses still remains controversial. The lack of understanding of selective replication of E1b55K-deleted Ads limits further development of the oncolytic virotherapy in cancer patients. Our goal is to fill these gaps and to obtain valuable information to benefit virotherapy. Previously we have demonstrated that cyclin E plays a critical role in adenovirus replication and deregulation of cyclin $\mathrm{E}$ expression in cancer cells is the molecular basis of selective replication of the oncolytic Ads. In this dissertation we further develop the mechanism model and extended the knowledge achieved from this model to the virtual application in the design of a novel tumor-specific oncolytic Ad.

Initial studies, shown in Chapter II, have demonstrated the role of cyclin E participates in oncolytic Ad replication through activation of CDK2; generating a suitable environment for viral replication. Ad E1B55K mediates cyclin E induction for viruses to efficiently replicate in normal cells. This E1B55K function is not critically required for the viral replication in cancer cells due to the deregulated cyclin E expression or having E1B55K-like cancer cellular factors, thus allowing 
preferential replication of $E 1 b 55 K$-deleted Ads in cancer cells. Ad-induced cyclin E binds to and activates CDK2 that subsequently phosphorylates the transcriptional suppressor $\mathrm{pRb}$, which can regulate expression of multiple cellular and viral genes, including cyclin E itself. (The work has been published in PLoS ONE 8(2): e57340.)

The work presented in Chapter III focuses on characterization of the replication profile of oncolytic virus Ad-cycE in human lung cancer cells. Cyclin E promoter is highly active in cancer cells and its activity is further enhanced after Ad infection. We utilized the dual properties of cyclin E promoter and have constructed a novel E1b-deleted Ad-cycE in which Ad E1A is driven by human cyclin E promoter as a novel therapeutic agent. Ad-cycE exhibits potent antitumor efficacy in vitro and in vivo and thus prolongs the survival time of mice bearing lung cancer xenograft. As cyclin $E$ is highly deregulated in numerous cancers and further augmented during virus multiplication, oncolytic Ad-cycE represents tumor specificity and has the possibility to overcome restriction in nonpermissive cancer cells for dl1520. (The manuscript is in preparation.)

In Chapter IV, we extended our studies from human lung cancer cells to evaluate the oncolytic efficacy of Ad-cycE with murine lung cancer cells. Our data showed that Ad-cycE can selectively replicate in and destroy murine ED-1 cancer cells derived from the mouse lung adenocarcinomas. Thus ED-1 is identified as a permissive murine lung cancer cell line for oncolytic Ad replication. We have 
evaluated the oncolytic efficacy of Ad-cycE with the syngeneic ED-1 mouse tumors in the immunocompetent FVB mice. (The manuscript is in preparation.)

As shown in Chapter $\mathrm{V}$, the impact of combination treatment with Ad-cycE and rapamycin which induces autophagy in lung cancer was explored based on the hypothesis that autophagy enhances oncolytic replication of Ad-cycE. Ad-cycE in combination with autophagy-inducer rapamycin has been shown to induce synergistic antitumor effects. Our data suggest this strategy involving in targeting cyclin E overexpression in cancer cells and enhanced autophagy could have a significant impact on clinical outcomes in cancer therapy. (The work has been published in Virology Journal 10(1):293)

The work presented in this dissertation has addressed the question, "By which mechanism does cyclin E contribute to oncolytic viral replication in cancer cells?" and revealed the potential of Ad-cycE for clinical application. Our work also raises a number of interesting directions for future research in the field of oncolytic virotherapy.

First, cancer cells with cyclin E deregulation may provide E1B55K-like factors to allow the cyclin $\mathrm{E}$ induction by E1b55K-deleted oncolytic Ads to overcome the growth restriction; however what kinds of cancer cellular factors existing in permissive cancer cells for this function are still unclear. Future study can focus on identifying the E1B55K-like factors existing in cancer cells and characterizing 
the following application to improve oncolytic virotherapy.

Second, our studies in the novel oncolytic Ad driven by cyclin E promoter have illuminated an attractive potential for this approach with dual properties to target both cancer tumorigenesis and virus-induced intracellular environment change. Transcriptionally controlling viral genes or other therapeutic genes with the human cyclin E promoter can be expected as feasible. Future vectors may combine this strategy with the other strategies, such as genetic modification on viral fibers or arming the virus with cytokine or anticancer genes. The new-generation tumor specific adenoviruses, with enhanced transduction efficiency and immunity stimulation, may increase cancer killing effects and improve therapeutic outcomes from oncolytic virotherapy.

Third, we have identified a novel permissive murine lung cancer cell line for oncolytic Ad replication. A future orthotopic lung cancer model in immunocompetent syngeneic mouse model should be developed using these murine lung cancer cells to better evaluate oncolytic virotherapy in a more patient-like setting and study the role of immune responses in oncolytic virotherapy. Further preclinical studies on pharmacodynamics, pharmacokinetics and the toxicity of oncolytic Ads with rodent and non-rodent models should also be investigated before Ad-cycE and the other similar Ad vectors can be applied to human clinical trials. 
Additionally, we observed the synergistic antitumor effects elicited by applying combination strategy with novel Ad-cycE and an autophagy inducer rapamycin in vitro. The future in vivo studies should be included to evaluate the benefit for clinical application. Another area of the field that bears further attention is to identify more potent autophagy inducers rather than only using the prototype drug rapamycin for this combination strategy. A screening test of combination effects of oncolytic Ads with novel autophagy inducers such as Spermidine (270), Saikosaponin-d (271) and GTM-1 (272) can be designed and conducted to select more efficient candidates to achieve improved outcomes. We believe that the future progression in oncolytic adenovirology, along with various improvements in therapeutic strategies should pave the way for the next wave of oncolytic virotherapy. 


\section{REFERENCES}

1. Siegel R, Naishadham D, Jemal A. Cancer statistics, 2013. CA Cancer J Clin. 2013;63:11-30.

2. Kirn D, Martuza RL, Zwiebel J. Replication-selective virotherapy for cancer: Biological principles, risk management and future directions. Nat Med. 2001;7:781-7.

3. Wang Y, Hallden G, Hill R, Anand A, Liu TC, Francis J, et al. E3 gene manipulations affect oncolytic adenovirus activity in immunocompetent tumor models. Nat Biotechnol. 2003;21:1328-35.

4. Parker JN, Bauer DF, Cody JJ, Markert JM. Oncolytic viral therapy of malignant glioma. Neurotherapeutics. 2009;6:558-69.

5. Russell SJ, Peng KW, Bell JC. Oncolytic virotherapy. Nat Biotechnol. 2012;30:658-70.

6. Nettelbeck DM. Cellular genetic tools to control oncolytic adenoviruses for virotherapy of cancer. J Mol Med (Berl). 2008;86:363-77.

7. Berk AJ. Adenoviridae: The Viruses and Their Replication. In: Howley DMKP, editor. Fields Virology. 5 ed. Philadelphia, PA: Lippincott Williams \& Wilkins; 2006. p. 2354-94.

8. Mulvihill S, Warren R, Venook A, Adler A, Randlev B, Heise C, et al. Safety and feasibility of injection with an E1B-55 $\mathrm{kDa}$ gene-deleted, replication-selective adenovirus (ONYX-015) into primary carcinomas of the pancreas: a phase I trial. Gene Ther. 2001;8:308-15.

9. Seth P. Adenoviral vectors. Adv Exp Med Biol. 2000;465:13-22.

10. Greber UF, Willetts M, Webster P, Helenius A. Stepwise dismantling of adenovirus 2 during entry into cells. Cell. 1993;75:477-86.

11. Davidson BL, Breakefield XO. Viral vectors for gene delivery to the nervous system. Nat Rev Neurosci. 2003;4:353-64.

12. Seth P. Mechanism of adenovirus-mediated endosome lysis: role of the intact adenovirus capsid structure. Biochem Biophys Res Commun. 1994;205:1318-24.

13. Lupold SE, Rodriguez R. Adenoviral gene therapy, radiation, and prostate cancer.

Rev Urol. 2005; 7:193-202.

14. Huebner RJ, Rowe WP, Schatten WE, Smith RR, Thomas LB. Studies on the use of viruses in the treatment of carcinoma of the cervix. Cancer. 1956;9:1211-8.

15. Barker DD, Berk AJ. Adenovirus proteins from both E1B reading frames are required for transformation of rodent cells by viral infection and DNA transfection. Virology. 1987;156:107-21.

16. Bischoff JR, Kirn DH, Williams A, Heise C, Horn S, Muna M, et al. An adenovirus mutant that replicates selectively in p53-deficient human tumor cells. Science. 1996;274:373-6.

17. Kirn D. Clinical research results with dl1520 (Onyx-015), a replication-selective adenovirus for the treatment of cancer: what have we learned? Gene therapy. 
2001;8:89-98.

18. Yu W, Fang H. Clinical trials with oncolytic adenovirus in China. Curr Cancer Drug Targets. 2007;7:141-8.

19. Lu W, Zheng S, Li XF, Huang JJ, Zheng X, Li Z. Intra-tumor injection of H101, a recombinant adenovirus, in combination with chemotherapy in patients with advanced cancers: a pilot phase II clinical trial. World J Gastroenterol. 2004;10:3634-8.

20. Li JL, Liu HL, Zhang XR, Xu JP, Hu WK, Liang M, et al. A phase I trial of intratumoral administration of recombinant oncolytic adenovirus overexpressing HSP70 in advanced solid tumor patients. Gene Ther. 2009;16:376-82.

21. Garber K. China approves world's first oncolytic virus therapy for cancer treatment. J Natl Cancer Inst. 2006;98:298-300.

22. Crompton AM, Kirn DH. From ONYX-015 to armed vaccinia viruses: the education and evolution of oncolytic virus development. Curr Cancer Drug Targets. 2007;7:133-9. 23. Frew SE, Sammut SM, Shore AF, Ramjist JK, Al-Bader S, Rezaie R, et al. Chinese health biotech and the billion-patient market. Nat Biotechnol. 2008;26:37-53.

24. Benjamin R, Helman L, Meyers P, Reaman G. A phase I/II dose escalation and activity study of intravenous injections of OCaP1 for subjects with refractory osteosarcoma metastatic to lung. Hum Gene Ther. 2001;12:1591-3.

25. Freytag SO, Khil M, Stricker H, Peabody J, Menon M, DePeralta-Venturina M, et al. Phase I study of replication-competent adenovirus-mediated double suicide gene therapy for the treatment of locally recurrent prostate cancer. Cancer Res. 2002;62:4968-76. 26. Freytag SO, Stricker H, Pegg J, Paielli D, Pradhan DG, Peabody J, et al. Phase I study of replication-competent adenovirus-mediated double-suicide gene therapy in combination with conventional-dose three-dimensional conformal radiation therapy for the treatment of newly diagnosed, intermediate- to high-risk prostate cancer. Cancer Res. 2003;63:7497-506.

27. Kimball KJ, Preuss MA, Barnes MN, Wang M, Siegal GP, Wan W, et al. A phase I study of a tropism-modified conditionally replicative adenovirus for recurrent malignant gynecologic diseases. Clin Cancer Res. 2010;16:5277-87.

28. Page JG, Tian B, Schweikart K, Tomaszewski J, Harris R, Broadt T, et al. Identifying the safety profile of a novel infectivity-enhanced conditionally replicative adenovirus, Ad5-delta24-RGD, in anticipation of a phase I trial for recurrent ovarian cancer. Am J Obstet Gynecol. 2007;196:389 e1-9; discussion e9-10.

29. DeWeese TL, van der Poel H, Li S, Mikhak B, Drew R, Goemann M, et al. A phase I trial of CV706, a replication-competent, PSA selective oncolytic adenovirus, for the treatment of locally recurrent prostate cancer following radiation therapy. Cancer Res. 2001;61:7464-72.

30. Small EJ, Carducci MA, Burke JM, Rodriguez R, Fong L, van Ummersen L, et al. A phase I trial of intravenous CG7870, a replication-selective, prostate-specific antigen-targeted oncolytic adenovirus, for the treatment of hormone-refractory, metastatic prostate cancer. Mol Ther. 2006;14:107-17.

31. Burke JM, Lamm DL, Meng MV, Nemunaitis JJ, Stephenson JJ, Arseneau JC, et al. A first in human phase 1 study of CG0070, a GM-CSF expressing oncolytic adenovirus, for the treatment of nonmuscle invasive bladder cancer. J Urol. 2012;188:2391-7. 32. Nemunaitis J, Tong AW, Nemunaitis M, Senzer N, Phadke AP, Bedell C, et al. A phase I study of telomerase-specific replication competent oncolytic adenovirus 
(telomelysin) for various solid tumors. Mol Ther. 2010;18:429-34.

33. Kanerva A, Nokisalmi P, Diaconu I, Koski A, Cerullo V, Liikanen I, et al. Antiviral and antitumor T-cell immunity in patients treated with GM-CSF-coding oncolytic adenovirus. Clin Cancer Res. 2013;19:2734-44.

34. Chang J, Zhao X, Wu X, Guo Y, Guo H, Cao J, et al. A Phase I study of KH901, a conditionally replicating granulocyte-macrophage colony-stimulating factor: armed oncolytic adenovirus for the treatment of head and neck cancers. Cancer Biol Ther. 2009;8:676-82.

35. Working PK, Lin A, Borellini F. Meeting product development challenges in manufacturing clinical grade oncolytic adenoviruses. Oncogene. 2005;24:7792-801. 36. Heise C, Sampson-Johannes A, Williams A, McCormick F, Von Hoff DD, Kirn DH. ONYX-015, an E1B gene-attenuated adenovirus, causes tumor-specific cytolysis and antitumoral efficacy that can be augmented by standard chemotherapeutic agents. Nat Med. 1997;3:639-45.

37. Ben-Israel H, Kleinberger T. Adenovirus and cell cycle control. Front Biosci. 2002;7:d1369-95.

38. Rao XM, Tseng MT, Zheng X, Dong Y, Jamshidi-Parsian A, Thompson TC, et al. E1A-induced apoptosis does not prevent replication of adenoviruses with deletion of E1b in majority of infected cancer cells. Cancer Gene Ther. 2004;11:585-93.

39. Amundson SA, Myers TG, Fornace AJ, Jr. Roles for p53 in growth arrest and apoptosis: putting on the brakes after genotoxic stress. Oncogene. 1998; 17:3287-99.

40. Braithwaite A, Nelson C, Skulimowski A, McGovern J, Pigott D, Jenkins J.

Transactivation of the p53 oncogene by E1a gene products. Virology. 1990;177:595-605. 41. Lowe SW, Ruley HE. Stabilization of the p53 tumor suppressor is induced by adenovirus 5 E1A and accompanies apoptosis. Genes Dev. 1993;7:535-45.

42. Nakajima T, Morita K, Tsunoda H, Imajoh-Ohmi S, Tanaka H, Yasuda H, et al. Stabilization of p53 by adenovirus E1A occurs through its amino-terminal region by modification of the ubiquitin-proteasome pathway. J Biol Chem. 1998;273:20036-45. 43. Pomerantz J, Schreiber-Agus N, Liegeois NJ, Silverman A, Alland L, Chin L, et al. The Ink4a tumor suppressor gene product, p19Arf, interacts with MDM2 and neutralizes MDM2's inhibition of p53. Cell. 1998;92:713-23.

44. Kao CC, Yew PR, Berk AJ. Domains required for in vitro association between the cellular p53 and the adenovirus 2 E1B 55K proteins. Virology. 1990;179:806-14. 45. Martin ME, Berk AJ. Adenovirus E1B 55K represses p53 activation in vitro. J Virol. 1998;72:3146-54.

46. Nevels M, Rubenwolf S, Spruss T, Wolf H, Dobner T. The adenovirus E4orf6 protein can promote E1 A/E1B-induced focus formation by interfering with p53 tumor suppressor function. Proc Natl Acad Sci U S A. 1997;94:1206-11.

47. Querido E, Blanchette P, Yan Q, Kamura T, Morrison M, Boivin D, et al.

Degradation of $\mathrm{p} 53$ by adenovirus E4orf6 and E1B55K proteins occurs via a novel mechanism involving a Cullin-containing complex. Genes Dev. 2001;15:3104-17. 48. Wienzek S, Roth J, Dobbelstein M. E1B 55-kilodalton oncoproteins of adenovirus types 5 and 12 inactivate and relocalize p53, but not p51 or p73, and cooperate with E4orf6 proteins to destabilize p53. J Virol. 2000;74:193-202.

49. Pennella MA, Liu Y, Woo JL, Kim CA, Berk AJ. Adenovirus E1B 55-kilodalton protein is a p53-SUMO1 E3 ligase that represses p53 and stimulates its nuclear export 
through interactions with promyelocytic leukemia nuclear bodies. J Virol.

2010;84:12210-25.

50. Hollstein M, Sidransky D, Vogelstein B, Harris CC. p53 mutations in human cancers. Science. 1991;253:49-53.

51. Pellegata NS, Ranzani GN. The significance of p53 mutations in human cancers. Eur J Histochem. 1996;40:273-82.

52. Dix BR, Edwards SJ, Braithwaite AW. Does the antitumor adenovirus ONYX-015/d11520 selectively target cells defective in the p53 pathway? J Virol. 2001;75:5443-7.

53. Goodrum FD, Ornelles DA. p53 status does not determine outcome of E1B 55-kilodalton mutant adenovirus lytic infection. J Virol. 1998;72:9479-90.

54. Geoerger B, Grill J, Opolon P, Morizet J, Aubert G, Terrier-Lacombe MJ, et al. Oncolytic activity of the E1B-55 kDa-deleted adenovirus ONYX-015 is independent of cellular p53 status in human malignant glioma xenografts. Cancer Res. 2002;62:764-72. 55. Rothmann T, Hengstermann A, Whitaker NJ, Scheffner M, zur Hausen H.

Replication of ONYX-015, a potential anticancer adenovirus, is independent of p53 status in tumor cells. J Virol. 1998;72:9470-8.

56. Zheng X, Rao XM, Gomez-Gutierrez JG, Hao H, McMasters KM, Zhou HS.

Adenovirus E1B55K region is required to enhance cyclin E expression for efficient viral DNA replication. J Virol. 2008;82:3415-27.

57. Ries SJ, Brandts CH, Chung AS, Biederer CH, Hann BC, Lipner EM, et al. Loss of p14ARF in tumor cells facilitates replication of the adenovirus mutant d11520 (ONYX-015). Nat Med. 2000;6:1128-33.

58. McCormick F. ONYX-015 selectivity and the p14ARF pathway. Oncogene. 2000;19:6670-2.

59. Edwards SJ, Dix BR, Myers CJ, Dobson-Le D, Huschtscha L, Hibma M, et al. Evidence that replication of the antitumor adenovirus ONYX-015 is not controlled by the p53 and p14(ARF) tumor suppressor genes. J Virol. 2002;76:12483-90.

60. Dix BR, O'Carroll SJ, Myers CJ, Edwards SJ, Braithwaite AW. Efficient induction of cell death by adenoviruses requires binding of E1B55k and p53. Cancer Res. 2000;60:2666-72.

61. Royds JA, Hibma M, Dix BR, Hananeia L, Russell IA, Wiles A, et al. p53 promotes adenoviral replication and increases late viral gene expression. Oncogene. 2006;25:1509-20.

62. Hobom U, Dobbelstein M. E1B-55-kilodalton protein is not required to block p53-induced transcription during adenovirus infection. J Virol. 2004;78:7685-97.

63. O'Shea CC, Johnson L, Bagus B, Choi S, Nicholas C, Shen A, et al. Late viral RNA export, rather than p53 inactivation, determines ONYX-015 tumor selectivity. Cancer Cell. 2004;6:611-23.

64. Flint SJ, Gonzalez RA. Regulation of mRNA production by the adenoviral E1B 55-kDa and E4 Orf6 proteins. Curr Top Microbiol Immunol. 2003;272:287-330.

65. Leppard KN, Shenk T. The adenovirus E1B $55 \mathrm{kd}$ protein influences mRNA transport via an intranuclear effect on RNA metabolism. EMBO J. 1989;8:2329-36. 66. Pilder S, Moore M, Logan J, Shenk T. The adenovirus E1B-55K transforming polypeptide modulates transport or cytoplasmic stabilization of viral and host cell mRNAs. Mol Cell Biol. 1986;6:470-6. 
67. Goodrum FD, Ornelles DA. Roles for the E4 orf6, orf3, and E1B 55-kilodalton proteins in cell cycle-independent adenovirus replication. J Virol. 1999;73:7474-88. 68. Woo JL, Berk AJ. Adenovirus ubiquitin-protein ligase stimulates viral late mRNA nuclear export. J Virol. 2007;81:575-87.

69. Kindsmuller K, Groitl P, Hartl B, Blanchette P, Hauber J, Dobner T. Intranuclear targeting and nuclear export of the adenovirus E1B-55K protein are regulated by SUMO1 conjugation. Proc Natl Acad Sci U S A. 2007;104:6684-9.

70. Schmid M, Kindsmuller K, Wimmer P, Groitl P, Gonzalez RA, Dobner T. The E3 ubiquitin ligase activity associated with the adenoviral E1B-55K-E4orf6 complex does not require CRM1-dependent export. J Virol. 2011;85:7081-94.

71. Blanchette P, Kindsmuller K, Groitl P, Dallaire F, Speiseder T, Branton PE, et al. Control of mRNA export by adenovirus E4orf6 and E1B55K proteins during productive infection requires E4orf6 ubiquitin ligase activity. J Virol. 2008;82:2642-51.

72. O'Shea CC, Soria C, Bagus B, McCormick F. Heat shock phenocopies E1B-55K late functions and selectively sensitizes refractory tumor cells to ONYX-015 oncolytic viral therapy. Cancer Cell. 2005;8:61-74.

73. Cuesta R, Xi Q, Schneider RJ. Adenovirus-specific translation by displacement of kinase Mnk1 from cap-initiation complex eIF4F. EMBO J. 2000;19:3465-74.

74. Cuesta R, Xi Q, Schneider RJ. Structural basis for competitive inhibition of eIF4G-Mnk1 interaction by the adenovirus 100-kilodalton protein. J Virol. 2004;78:7707-16.

75. Hayes BW, Telling GC, Myat MM, Williams JF, Flint SJ. The adenovirus L4 100-kilodalton protein is necessary for efficient translation of viral late mRNA species. $\mathrm{J}$ Virol. 1990;64:2732-42.

76. Yueh A, Schneider RJ. Translation by ribosome shunting on adenovirus and hsp70 mRNAs facilitated by complementarity to 18S rRNA. Genes Dev. 2000;14:414-21.

77. Gonzalez R, Huang W, Finnen R, Bragg C, Flint SJ. Adenovirus E1B 55-kilodalton protein is required for both regulation of mRNA export and efficient entry into the late phase of infection in normal human fibroblasts. J Virol. 2006;80:964-74.

78. Goodrum FD, Ornelles DA. The early region 1B 55-kilodalton oncoprotein of adenovirus relieves growth restrictions imposed on viral replication by the cell cycle. J Virol. 1997;71:548-61.

79. Rao XM, Zheng X, Waigel S, Zacharias W, McMasters KM, Zhou HS. Gene expression profiles of normal human lung cells affected by adenoviral E1B. Virology. 2006;350:418-28.

80. Ohtsubo M, Theodoras AM, Schumacher J, Roberts JM, Pagano M. Human cyclin E, a nuclear protein essential for the G1-to-S phase transition. Mol Cell Biol. 1995;15:2612-24.

81. Le Cam L, Polanowska J, Fabbrizio E, Olivier M, Philips A, Ng Eaton E, et al. Timing of cyclin $\mathrm{E}$ gene expression depends on the regulated association of a bipartite repressor element with a novel E2F complex. EMBO J. 1999;18:1878-90.

82. Moroy T, Geisen C. Cyclin E. Int J Biochem Cell Biol. 2004;36:1424-39.

83. Geng Y, Lee YM, Welcker M, Swanger J, Zagozdzon A, Winer JD, et al.

Kinase-independent function of cyclin E. Mol Cell. 2007;25:127-39.

84. Geisen C, Moroy T. The oncogenic activity of cyclin E is not confined to Cdk2 activation alone but relies on several other, distinct functions of the protein. J Biol Chem. 
2002;277:39909-18.

85. Hwang HC, Clurman BE. Cyclin E in normal and neoplastic cell cycles. Oncogene. 2005;24:2776-86.

86. Loeb KR, Kostner H, Firpo E, Norwood T, K DT, Clurman BE, et al. A mouse model for cyclin E-dependent genetic instability and tumorigenesis. Cancer Cell. 2005;8:35-47.

87. Donnellan R, Chetty R. Cyclin E in human cancers. FASEB J. 1999;13:773-80.

88. Ma Y, Fiering S, Black C, Liu X, Yuan Z, Memoli VA, et al. Transgenic cyclin E triggers dysplasia and multiple pulmonary adenocarcinomas. Proc Natl Acad Sci U S A. 2007;104:4089-94.

89. Spruck CH, Won KA, Reed SI. Deregulated cyclin E induces chromosome instability. Nature. 1999;401:297-300.

90. Minella AC, Swanger J, Bryant E, Welcker M, Hwang H, Clurman BE. p53 and p21

form an inducible barrier that protects cells against cyclin E-cdk2 deregulation. Curr Biol. 2002;12:1817-27.

91. Marone M, Scambia G, Giannitelli C, Ferrandina G, Masciullo V, Bellacosa A, et al. Analysis of cyclin E and CDK2 in ovarian cancer: gene amplification and RNA overexpression. Int J Cancer. 1998;75:34-9.

92. Sawasaki T, Shigemasa K, Shiroyama Y, Kusuda T, Fujii T, Parmley TH, et al.

Cyclin E mRNA overexpression in epithelial ovarian cancers: inverse correlation with p53 protein accumulation. J Soc Gynecol Investig. 2001;8:179-85.

93. Strohmaier H, Spruck CH, Kaiser P, Won KA, Sangfelt O, Reed SI. Human F-box protein hCdc4 targets cyclin $\mathrm{E}$ for proteolysis and is mutated in a breast cancer cell line. Nature. 2001;413:316-22.

94. Akli S, Zheng PJ, Multani AS, Wingate HF, Pathak S, Zhang N, et al.

Tumor-specific low molecular weight forms of cyclin $\mathrm{E}$ induce genomic instability and resistance to p21, p27, and antiestrogens in breast cancer. Cancer Res. 2004;64:3198-208. 95. Keyomarsi K, Tucker SL, Buchholz TA, Callister M, Ding Y, Hortobagyi GN, et al. Cyclin E and survival in patients with breast cancer. N Engl J Med. 2002;347:1566-75. 96. Wingate H, Puskas A, Duong M, Bui T, Richardson D, Liu Y, et al. Low molecular weight cyclin $\mathrm{E}$ is specific in breast cancer and is associated with mechanisms of tumor progression. Cell Cycle. 2009;8:1062-8.

97. Shenk T. Adenoviridae: the viruses and their replication. In: Knipe DM, Howley PM, editors. Fields Virology. 4 ed. Philadelphia, PA: Lippincott Williams \& Wilkins; 2001.p. 2265-300.

98. Avvakumov N, Sahbegovic M, Zhang Z, Shuen M, Mymryk JS. Analysis of DNA binding by the adenovirus type 5 E1A oncoprotein. J Gen Virol. 2002;83:517-24.

99. Dyson N, Harlow E. Adenovirus E1 A targets key regulators of cell proliferation. Cancer Surv. 1992;12:161-95.

100. Ludlow JW, Skuse GR. Viral oncoprotein binding to pRB, p107, p130, and p300. Virus Res. 1995;35:113-21.

101. Whyte P, Buchkovich KJ, Horowitz JM, Friend SH, Raybuck M, Weinberg RA, et al. Association between an oncogene and an anti-oncogene: the adenovirus E1A proteins bind to the retinoblastoma gene product. Nature. 1988;334:124-9.

102. Liu Y, Costantino ME, Montoya-Durango D, Higashi Y, Darling DS, Dean DC. The zinc finger transcription factor ZFHX1A is linked to cell proliferation by Rb-E2F1. 
Biochem J. 2007;408:79-85.

103. Rowland BD, Denissov SG, Douma S, Stunnenberg HG, Bernards R, Peeper DS.

E2F transcriptional repressor complexes are critical downstream targets of p19(ARF)/p53-induced proliferative arrest. Cancer Cell. 2002;2:55-65.

104. Zhang HS, Postigo AA, Dean DC. Active transcriptional repression by the Rb-E2F complex mediates G1 arrest triggered by p16INK4a, TGFbeta, and contact inhibition.

Cell. 1999;97:53-61.

105. Pelka P, Miller MS, Cecchini M, Yousef AF, Bowdish DM, Dick F, et al. Adenovirus E1A directly targets the E2F/DP-1 complex. J Virol. 2011;85:8841-51.

106. Debbas M, White E. Wild-type p53 mediates apoptosis by E1A, which is inhibited by E1B. Genes Dev. 1993;7:546-54.

107. Querido E, Marcellus RC, Lai A, Charbonneau R, Teodoro JG, Ketner G, et al. Regulation of p53 levels by the E1B 55-kilodalton protein and E4orf6 in adenovirus-infected cells. J Virol. 1997;71:3788-98.

108. Yew PR, Berk AJ. Inhibition of p53 transactivation required for transformation by adenovirus early 1B protein. Nature. 1992;357:82-5.

109. Rogulski KR, Freytag SO, Zhang K, Gilbert JD, Paielli DL, Kim JH, et al. In vivo antitumor activity of ONYX-015 is influenced by p53 status and is augmented by radiotherapy. Cancer Res. 2000;60:1193-6.

110. Coverley D, Laman H, Laskey RA. Distinct roles for cyclins E and A during DNA replication complex assembly and activation. Nat Cell Biol. 2002;4:523-8.

111. Furstenthal L, Kaiser BK, Swanson C, Jackson PK. Cyclin E uses Cdc6 as a chromatin-associated receptor required for DNA replication. J Cell Biol.

2001;152:1267-78.

112. Hinchcliffe EH, Li C, Thompson EA, Maller JL, Sluder G. Requirement of Cdk2-cyclin E activity for repeated centrosome reproduction in Xenopus egg extracts. Science. 1999;283:851-4.

113. Matsumoto Y, Hayashi K, Nishida E. Cyclin-dependent kinase 2 (Cdk2) is required for centrosome duplication in mammalian cells. Curr Biol. 1999;9:429-32.

114. Bortner DM, Rosenberg MP. Induction of mammary gland hyperplasia and carcinomas in transgenic mice expressing human cyclin E. Mol Cell Biol. 1997;17:453-9. 115. Freemantle SJ, Dmitrovsky E. Cyclin E transgenic mice: discovery tools for lung cancer biology, therapy, and prevention. Cancer Prev Res (Phila). 2010;3:1513-8.

116. Ortega S, Prieto I, Odajima J, Martin A, Dubus P, Sotillo R, et al. Cyclin-dependent kinase 2 is essential for meiosis but not for mitotic cell division in mice. Nat Genet.

2003;35:25-31.

117. Berthet C, Aleem E, Coppola V, Tessarollo L, Kaldis P. Cdk2 knockout mice are viable. Curr Biol. 2003;13:1775-85.

118. Geng Y, Yu Q, Sicinska E, Das M, Schneider JE, Bhattacharya S, et al. Cyclin E ablation in the mouse. Cell. 2003;114:431-43.

119. Matsumoto Y, Maller JL. A centrosomal localization signal in cyclin E required for Cdk2-independent S phase entry. Science. 2004;306:885-8.

120. Sandig V, Youil R, Bett AJ, Franlin LL, Oshima M, Maione D, et al. Optimization of the helper-dependent adenovirus system for production and potency in vivo. Proc Natl Acad Sci U S A. 2000;97:1002-7.

121. Zhao T, Rao XM, Xie X, Li L, Thompson TC, McMasters KM, et al. Adenovirus 
with insertion-mutated E1A selectively propagates in liver cancer cells and destroys tumors in vivo. Cancer Res. 2003;63:3073-8.

122. Bett AJ, Haddara W, Prevec L, Graham FL. An efficient and flexible system for construction of adenovirus vectors with insertions or deletions in early regions 1 and 3 . Proc Natl Acad Sci U S A. 1994;91:8802-6.

123. Zheng X, Rao XM, Snodgrass CL, McMasters KM, Zhou HS. Selective replication of E1B55K-deleted adenoviruses depends on enhanced E1A expression in cancer cells. Cancer Gene Ther. 2006;13:572-83.

124. Chou MM, Masuda-Robens JM, Gupta ML. Cdc42 promotes G1 progression through p70 S6 kinase-mediated induction of cyclin E expression. J Biol Chem. 2003;278:35241-7.

125. Gu Y, Rosenblatt J, Morgan DO. Cell cycle regulation of CDK2 activity by phosphorylation of Thr160 and Tyr15. EMBO J. 1992;11:3995-4005.

126. Hunter T, Pines J. Cyclins and cancer. II: Cyclin D and CDK inhibitors come of age. Cell. 1994;79:573-82.

127. Sherr CJ. D-type cyclins. Trends Biochem Sci. 1995;20:187-90.

128.Moldovan GL, Pfander B, Jentsch S. PCNA, the maestro of the replication fork. Cell. 2007;129:665-79.

129. Zhang H, Xiong Y, Beach D. Proliferating cell nuclear antigen and $\mathrm{p} 21$ are components of multiple cell cycle kinase complexes. Mol Biol Cell. 1993;4:897-906.

130. Zarkowska T, Mittnacht S. Differential phosphorylation of the retinoblastoma protein by G1/S cyclin-dependent kinases. J Biol Chem. 1997;272:12738-46.

131. Schmitz NM, Leibundgut K, Hirt A. CDK2 catalytic activity and loss of nuclear tethering of retinoblastoma protein in childhood acute lymphoblastic leukemia. Leukemia. 2005;19:1783-7.

132. Hsieh WS, Soo R, Peh BK, Loh T, Dong D, Soh D, et al. Pharmacodynamic effects of seliciclib, an orally administered cell cycle modulator, in undifferentiated nasopharyngeal cancer. Clin Cancer Res. 2009;15:1435-42.

133. Connell-Crowley L, Harper JW, Goodrich DW. Cyclin D1/Cdk4 regulates retinoblastoma protein-mediated cell cycle arrest by site-specific phosphorylation. Mol Biol Cell. 1997;8:287-301.

134. Sherr CJ, Roberts JM. Inhibitors of mammalian G1 cyclin-dependent kinases. Genes Dev. 1995;9:1149-63.

135. De Azevedo WF, Leclerc S, Meijer L, Havlicek L, Strnad M, Kim SH. Inhibition of cyclin-dependent kinases by purine analogues: crystal structure of human cdk2 complexed with roscovitine. Eur J Biochem. 1997;243:518-26. 136. Raynaud FI, Whittaker SR, Fischer PM, McClue S, Walton MI, Barrie SE, et al. In vitro and in vivo pharmacokinetic-pharmacodynamic relationships for the trisubstituted aminopurine cyclin-dependent kinase inhibitors olomoucine, bohemine and CYC202. Clin Cancer Res. 2005;11:4875-87.

137. Akli S, Bui T, Wingate H, Biernacka A, Moulder S, Tucker SL, et al.

Low-molecular-weight cyclin E can bypass letrozole-induced G1 arrest in human breast cancer cells and tumors. Clin Cancer Res. 2010;16:1179-90.

138. Meijer L, Borgne A, Mulner O, Chong JP, Blow JJ, Inagaki N, et al. Biochemical and cellular effects of roscovitine, a potent and selective inhibitor of the cyclin-dependent kinases cdc2, cdk2 and cdk5. Eur J Biochem. 1997;243:527-36. 
139. Hayflick L, Plotkin SA, Norton TW, Koprowski H. Preparation of poliovirus vaccines in a human fetal diploid cell strain. Am J Hyg. 1962;75:240-58. 140. Keyomarsi K, O'Leary N, Molnar G, Lees E, Fingert HJ, Pardee AB. Cyclin E, a potential prognostic marker for breast cancer. Cancer Res. 1994;54:380-5.

141. Porter DC, Zhang N, Danes C, McGahren MJ, Harwell RM, Faruki S, et al. Tumor-specific proteolytic processing of cyclin $\mathrm{E}$ generates hyperactive lower-molecular-weight forms. Mol Cell Biol. 2001;21:6254-69.

142. Koff A, Giordano A, Desai D, Yamashita K, Harper JW, Elledge S, et al. Formation and activation of a cyclin E-cdk2 complex during the G1 phase of the human cell cycle. Science. 1992;257:1689-94.

143. Harwell RM, Mull BB, Porter DC, Keyomarsi K. Activation of cyclin-dependent kinase 2 by full length and low molecular weight forms of cyclin $\mathrm{E}$ in breast cancer cells. J Biol Chem. 2004;279:12695-705.

144. Weinberg RA. The retinoblastoma protein and cell cycle control. Cell. 1995;81:323-30.

145. Giacinti C, Giordano A. RB and cell cycle progression. Oncogene. 2006;25:5220-7. 146. Ohtani K, DeGregori J, Nevins JR. Regulation of the cyclin E gene by transcription factor E2F1. Proc Natl Acad Sci U S A. 1995;92:12146-50.

147. Duronio RJ, O'Farrell PH. Developmental control of the G1 to $S$ transition in Drosophila: cyclin Eis a limiting downstream target of E2F. Genes Dev. 1995;9:1456-68. 148. Sheaff RJ, Groudine M, Gordon M, Roberts JM, Clurman BE. Cyclin E-CDK2 is a regulator of p27Kip1. Genes Dev. 1997;11:1464-78.

149. Zhu H, Nie L, Maki CG. Cdk2-dependent Inhibition of p21 stability via a C-terminal cyclin-binding motif. J Biol Chem. 2005;280:29282-8.

150. Vlach J, Hennecke S, Amati B. Phosphorylation-dependent degradation of the cyclin-dependent kinase inhibitor p27. EMBO J. 1997;16:5334-44.

151. Montagnoli A, Fiore F, Eytan E, Carrano AC, Draetta GF, Hershko A, et al. Ubiquitination of $\mathrm{p} 27$ is regulated by Cdk-dependent phosphorylation and trimeric complex formation. Genes Dev. 1999;13:1181-9.

152. Bornstein G, Bloom J, Sitry-Shevah D, Nakayama K, Pagano M, Hershko A. Role of the SCFSkp2 ubiquitin ligase in the degradation of p21Cip1 in S phase. J Biol Chem. 2003;278:25752-7.

153. Wold WS, Toth K. Chapter three--Syrian hamster as an animal model to study oncolytic adenoviruses and to evaluate the efficacy of antiviral compounds. Adv Cancer Res. 2012;115:69-92.

154. Vollmer CM, Ribas A, Butterfield LH, Dissette VB, Andrews KJ, Eilber FC, et al. p53 selective and nonselective replication of an E1B-deleted adenovirus in hepatocellular carcinoma. Cancer Res. 1999;59:4369-74.

155. Toth K, Dhar D, Wold WS. Oncolytic (replication-competent) adenoviruses as anticancer agents. Expert Opin Biol Ther. 2010;10:353-68.

156. Kurihara T, Brough DE, Kovesdi I, Kufe DW. Selectivity of a replication-competent adenovirus for human breast carcinoma cells expressing the MUC1 antigen. J Clin Invest. 2000;106:763-71.

157. Dong X, Qu W, Ma S, Zhu Z, Zheng C, He A, et al. Potent antitumoral effects of targeted promoter-driven oncolytic adenovirus armed with Dm-dNK for breast cancer in vitro and in vivo. Cancer Lett. 2013;328:95-103. 
158. Cafferata EG, Maccio DR, Lopez MV, Viale DL, Carbone C, Mazzolini G, et al. A novel A33 promoter-based conditionally replicative adenovirus suppresses tumor growth and eradicates hepatic metastases in human colon cancer models. Clinical cancer research : an official journal of the American Association for Cancer Research. 2009;15:3037-49.

159. Fu X, Meng F, Tao L, Jin A, Zhang X. A strict-late viral promoter is a strong tumor-specific promoter in the context of an oncolytic herpes simplex virus. Gene Ther. 2003;10:1458-64.

160. Guo ZS, Thorne SH, Bartlett DL. Oncolytic virotherapy: molecular targets in tumor-selective replication and carrier cell-mediated delivery of oncolytic viruses. Biochim Biophys Acta. 2008;1785:217-31.

161. Davis JJ, Wang L, Dong F, Zhang L, Guo W, Teraishi F, et al. Oncolysis and suppression of tumor growth by a GFP-expressing oncolytic adenovirus controlled by an hTERT and CMV hybrid promoter. Cancer Gene Ther. 2006;13:720-3.

162. Ji X, Zhang J, Cheng L, Wei F, Li H, Liu X, et al. Oncolytic adenovirus delivering herpes simplex virus thymidine kinase suicide gene reduces the growth of human retinoblastoma in an in vivo mouse model. Exp Eye Res. 2009;89:193-9.

163. Xie X, Xia W, Li Z, Kuo HP, Liu Y, Ding Q, et al. Targeted expression of BikDD eradicates pancreatic tumors in noninvasive imaging models. Cancer Cell.

2007;12:52-65.

164. Cheng PH, Rao XM, McMasters KM, Zhou HS. Molecular basis for viral selective replication in cancer cells: activation of CDK2 by adenovirus-induced cyclin E. PLoS One. 2013;8:e57340.

165. Gomez-Gutierrez JG, Rao XM, Zhou HS, McMasters KM. Enhanced cancer cell killing by truncated E2F-1 used in combination with oncolytic adenovirus. Virology. 2012;433:538-47.

166. Rodriguez-Rocha H, Gomez-Gutierrez JG, Garcia-Garcia A, Rao XM, Chen L, McMasters KM, et al. Adenoviruses induce autophagy to promote virus replication and oncolysis. Virology. 2011;416:9-15.

167. Kakinuma H, Bergert ER, Spitzweg C, Cheville JC, Lieber MM, Morris JC. Probasin promoter (ARR(2)PB)-driven, prostate-specific expression of the human sodium iodide symporter (h-NIS) for targeted radioiodine therapy of prostate cancer. Cancer Res. 2003;63:7840-4.

168. Geng Y, Eaton EN, Picon M, Roberts JM, Lundberg AS, Gifford A, et al. Regulation of cyclin E transcription by E2Fs and retinoblastoma protein. Oncogene.

1996;12:1173-80.

169. Cheng PH, Lian S, Zhao R, Rao XM, McMasters KM, Zhou HS. Combination of autophagy inducer rapamycin and oncolytic adenovirus improves antitumor effect in cancer cells. Virol J. 2013;10:293.

170. Ishiyama M, Tominaga H, Shiga M, Sasamoto K, Ohkura Y, Ueno K. A combined assay of cell viability and in vitro cytotoxicity with a highly water-soluble tetrazolium salt, neutral red and crystal violet. Biol Pharm Bull. 1996;19:1518-20.

171. Kwon OJ, Kim PH, Huyn S, Wu L, Kim M, Yun CO. A hypoxia- and \{alpha\}-fetoprotein-dependent oncolytic adenovirus exhibits specific killing of hepatocellular carcinomas. Clin Cancer Res. 2010;16:6071-82.

172. Kurozumi K, Hardcastle J, Thakur R, Yang M, Christoforidis G, Fulci G, et al. Effect 
of tumor microenvironment modulation on the efficacy of oncolytic virus therapy. $\mathrm{J}$ Natl Cancer Inst. 2007;99:1768-81.

173. Huang T, Civelek AC, Zheng H, Ng CK, Duan X, Li J, et al. (18)F-misonidazole PET imaging of hypoxia in micrometastases and macroscopic xenografts of human non-small cell lung cancer: a correlation with autoradiography and histological findings. American journal of nuclear medicine and molecular imaging. 2013;3:142-53.

174. Hayflick L. The Limited in Vitro Lifetime of Human Diploid Cell Strains. Exp Cell Res. 1965;37:614-36.

175. Wadler S, Yu B, Tan JY, Kaleya R, Rozenblit A, Makower D, et al. Persistent replication of the modified chimeric adenovirus ONYX-015 in both tumor and stromal cells from a patient with gall bladder carcinoma implants. Clinical cancer research : an official journal of the American Association for Cancer Research. 2003;9:33-43. 176. Mullen JT, Tanabe KK. Viral oncolysis. Oncologist. 2002;7:106-19.

177. Kim J, Cho JY, Kim JH, Jung KC, Yun CO. Evaluation of E1B gene-attenuated replicating adenoviruses for cancer gene therapy. Cancer Gene Ther. 2002;9:725-36. 178. Kondo Y, Kanzawa T, Sawaya R, Kondo S. The role of autophagy in cancer development and response to therapy. Nature reviews Cancer. 2005;5:726-34.

179. Gozuacik D, Kimchi A. Autophagy as a cell death and tumor suppressor mechanism. Oncogene. 2004;23:2891-906.

180. Eager RM, Nemunaitis J. Clinical development directions in oncolytic viral therapy. Cancer Gene Ther. 2011;18:305-17.

181. Donnelly OG, Errington-Mais F, Prestwich R, Harrington K, Pandha H, Vile R, et al. Recent clinical experience with oncolytic viruses. Curr Pharm Biotechnol.

2012;13:1834-41.

182. Kruyt FA, Curiel DT. Toward a new generation of conditionally replicating adenoviruses: pairing tumor selectivity with maximal oncolysis. Hum Gene Ther. 2002;13:485-95.

183. Zhu ZB, Makhija SK, Lu B, Wang M, Rivera AA, Kim-Park S, et al. Incorporating the survivin promoter in an infectivity enhanced CRAd-analysis of oncolysis and anti-tumor effects in vitro and in vivo. Int J Oncol. 2005;27:237-46.

184. Wang H, Wei F, Zhang J, Wang F, Li H, Chen X, et al. A novel immunocompetent murine tumor model for the evaluation of RCAd-enhanced RDAd transduction efficacy. Tumour Biol. 2012;33:1245-53.

185. Wold WS, Hermiston TW, Tollefson AE. Adenovirus proteins that subvert host defenses. Trends Microbiol. 1994;2:437-43.

186. Hallden G, Hill R, Wang Y, Anand A, Liu TC, Lemoine NR, et al. Novel immunocompetent murine tumor models for the assessment of replication-competent oncolytic adenovirus efficacy. Mol Ther. 2003;8:412-24.

187. Liu X, Sempere LF, Galimberti F, Freemantle SJ, Black C, Dragnev KH, et al. Uncovering growth-suppressive MicroRNAs in lung cancer. Clin Cancer Res. 2009; 15:1177-83.

188. Fan W, Lin CS, Potluri P, Procaccio V, Wallace DC. mtDNA lineage analysis of mouse L-cell lines reveals the accumulation of multiple mtDNA mutants and intermolecular recombination. Genes Dev. 2012;26:384-94.

189. Widera D, Zander C, Heidbreder M, Kasperek Y, Noll T, Seitz O, et al. Adult palatum as a novel source of neural crest-related stem cells. Stem Cells. 
2009;27:1899-910.

190. Roth V. http://www.doubling-time.com/compute.php. 2006 [cited; Available from: 191. Nguyen MD, Lariviere RC, Julien JP. Reduction of axonal caliber does not alleviate motor neuron disease caused by mutant superoxide dismutase 1. Proc Natl Acad Sci U S A. 2000;97:12306-11.

192. Ganly I, Kim YT, Hann B, Balmain A, Brown R. Replication and cytolysis of an E1B-attenuated adenovirus in drug-resistant ovarian tumour cells is associated with reduced apoptosis. Gene Ther. 2001;8:369-75.

193. Ganly I, Mautner V, Balmain A. Productive replication of human adenoviruses in mouse epidermal cells. J Virol. 2000;74:2895-9.

194. Madara J, Krewet JA, Shah M. Heat shock protein 72 expression allows permissive replication of oncolytic adenovirus d11520 (ONYX-015) in rat glioblastoma cells. Mol Cancer. 2005;4:12.

195. Todaro GJ, Green H. Quantitative studies of the growth of mouse embryo cells in culture and their development into established lines. J Cell Biol. 1963;17:299-313. 196. Harlow E, Franza BR, Jr., Schley C. Monoclonal antibodies specific for adenovirus early region 1A proteins: extensive heterogeneity in early region 1A products. J Virol. 1985;55:533-46.

197. Zhang Y, Bergelson JM. Adenovirus receptors. J Virol. 2005;79:12125-31.

198. Bergelson JM, Cunningham JA, Droguett G, Kurt-Jones EA, Krithivas A, Hong JS, et al. Isolation of a common receptor for Coxsackie B viruses and adenoviruses 2 and 5. Science. 1997;275:1320-3.

199. Shayakhmetov DM, Li ZY, Ternovoi V, Gaggar A, Gharwan H, Lieber A. The interaction between the fiber knob domain and the cellular attachment receptor determines the intracellular trafficking route of adenoviruses. J Virol. 2003;77:3712-23. 200. Kirby I, Davison E, Beavil AJ, Soh CP, Wickham TJ, Roelvink PW, et al. Identification of contact residues and definition of the CAR-binding site of adenovirus type 5 fiber protein. J Virol. 2000;74:2804-13.

201. Wickham TJ, Mathias P, Cheresh DA, Nemerow GR. Integrins alpha v beta 3 and alpha $\mathrm{v}$ beta 5 promote adenovirus internalization but not virus attachment. Cell. 1993;73:309-19.

202. Nemerow GR, Stewart PL. Role of alpha(v) integrins in adenovirus cell entry and gene delivery. Microbiol Mol Biol Rev. 1999;63:725-34.

203. Tomko RP, Xu R, Philipson L. HCAR and MCAR: the human and mouse cellular receptors for subgroup $\mathrm{C}$ adenoviruses and group B coxsackieviruses. Proc Natl Acad Sci U S A. 1997;94:3352-6.

204. Li E, Brown SL, Stupack DG, Puente XS, Cheresh DA, Nemerow GR. Integrin alpha(v)beta1 is an adenovirus coreceptor. J Virol. 2001;75:5405-9.

205. Salone B, Martina Y, Piersanti S, Cundari E, Cherubini G, Franqueville L, et al. Integrin alpha3beta1 is an alternative cellular receptor for adenovirus serotype 5. J Virol. 2003;77:13448-54.

206. Arend LJ, Smart AM, Briggs JP. Mouse beta(6) integrin sequence, pattern of expression, and role in kidney development. J Am Soc Nephrol. 2000;11:2297-305. 207. Bengtsson T, Camper L, Schneller M, Lundgren-Akerlund E. Characterization of the mouse integrin subunit alpha10 gene and comparison with its human homologue.

Genomic structure, chromosomal localization and identification of splice variants. Matrix 
Biol. 2001;20:565-76.

208. Dechecchi MC, Melotti P, Bonizzato A, Santacatterina M, Chilosi M, Cabrini G. Heparan sulfate glycosaminoglycans are receptors sufficient to mediate the initial binding of adenovirus types 2 and 5. J Virol. 2001;75:8772-80.

209. Dechecchi MC, Tamanini A, Bonizzato A, Cabrini G. Heparan sulfate glycosaminoglycans are involved in adenovirus type 5 and 2-host cell interactions. Virology. 2000;268:382-90.

210. Hileman RE, Fromm JR, Weiler JM, Linhardt RJ. Glycosaminoglycan-protein interactions: definition of consensus sites in glycosaminoglycan binding proteins. Bioessays. 1998;20:156-67.

211. Raman S, Hsu TH, Ashley SL, Spindler KR. Usage of integrin and heparan sulfate as receptors for mouse adenovirus type 1. J Virol. 2009;83:2831-8.

212. Duncan SJ, Gordon FC, Gregory DW, McPhie JL, Postlethwaite R, White R, et al. Infection of mouse liver by human adenovirus type 5. J Gen Virol. 1978;40:45-61. 213. Biron KK, Raska K, Jr. Adenovirus type 12 infection of defined mouse-human hybrid cell clones. Experientia. 1976;32:38-40.

214. Eggerding FA, Pierce WC. Molecular biology of adenovirus type 2 semipermissive infections. I. Viral growth and expression of viral replicative functions during restricted adenovirus infection. Virology. 1986;148:97-113.

215. Blair GE, Dixon SC, Griffiths SA, Zajdel ME. Restricted replication of human adenovirus type 5 in mouse cell lines. Virus Res. 1989;14:339-46.

216. Lucher LA. Abortive adenovirus infection and host range determinants. Curr Top Microbiol Immunol. 1995;199 ( Pt 1):119-52.

217. Seth P, Rosenfeld M, Higginbotham J, Crystal RG. Mechanism of enhancement of DNA expression consequent to cointernalization of a replication-deficient adenovirus and unmodified plasmid DNA. J Virol. 1994;68:933-40.

218. Silverstein G, Strohl WA. Restricted replication of adenovirus type 2 in mouse Balb/3T3 cells. Arch Virol. 1986;87:241-64.

219. Zhang H. Life without kinase: cyclin E promotes DNA replication licensing and beyond. Mol Cell. 2007;25:175-6.

220. Kim KH, Ryan MJ, Estep JE, Miniard BM, Rudge TL, Peggins JO, et al. A new generation of serotype chimeric infectivity-enhanced conditionally replicative adenovirals: the safety profile of ad5/3-Delta24 in advance of a phase I clinical trial in ovarian cancer patients. Hum Gene Ther. 2011;22:821-8.

221. Waszak P, Franco-Montoya ML, Jacob MP, Deprez I, Levame M, Lafuma C, et al. Effect of intratracheal adenoviral vector administration on lung development in newborn rats. Hum Gene Ther. 2002;13:1873-85.

222. Ottolino-Perry K, Diallo JS, Lichty BD, Bell JC, McCart JA. Intelligent design: combination therapy with oncolytic viruses. Mol Ther. 2010;18:251-63.

223. Stromhaug PE, Berg TO, Fengsrud M, Seglen PO. Purification and characterization of autophagosomes from rat hepatocytes. Biochem J. 1998;335 ( Pt 2):217-24.

224. Chen Y, Azad MB, Gibson SB. Methods for detecting autophagy and determining autophagy-induced cell death. Canadian journal of physiology and pharmacology. 2010;88:285-95.

225. Kabeya Y, Mizushima N, Ueno T, Yamamoto A, Kirisako T, Noda T, et al. LC3, a mammalian homologue of yeast Apg8p, is localized in autophagosome membranes after 
processing. EMBO J. 2000;19:5720-8.

226. Mizushima N. Methods for monitoring autophagy. Int J Biochem Cell Biol. 2004;36:2491-502.

227. Mizushima N, Yoshimori T. How to interpret LC3 immunoblotting. Autophagy. 2007;3:542-5.

228. Kim HJ, Lee S, Jung JU. When autophagy meets viruses: a double-edged sword with functions in defense and offense. Seminars in immunopathology. 2010;32:323-41. 229. Barth S, Glick D, Macleod KF. Autophagy: assays and artifacts. The Journal of pathology. 2010;221:117-24.

230. Sarbassov DD, Ali SM, Sabatini DM. Growing roles for the mTOR pathway. Curr Opin Cell Biol. 2005;17:596-603.

231. Sarbassov DD, Ali SM, Sengupta S, Sheen JH, Hsu PP, Bagley AF, et al. Prolonged rapamycin treatment inhibits mTORC2 assembly and Akt/PKB. Mol Cell. 2006;22:159-68.

232. Takeuchi H, Kondo Y, Fujiwara K, Kanzawa T, Aoki H, Mills GB, et al. Synergistic augmentation of rapamycin-induced autophagy in malignant glioma cells by phosphatidylinositol 3-kinase/protein kinase B inhibitors. Cancer Res. 2005;65:3336-46. 233. Khanna S, Darbre PD. Parabens enable suspension growth of MCF-10A immortalized, non-transformed human breast epithelial cells. Journal of applied toxicology : JAT. 2013;33:378-82.

234. Soule HD, Maloney TM, Wolman SR, Peterson WD, Jr., Brenz R, McGrath CM, et al. Isolation and characterization of a spontaneously immortalized human breast epithelial cell line, MCF-10. Cancer Res. 1990;50:6075-86.

235. Wang S, Meckling KA, Marcone MF, Kakuda Y, Tsao R. Synergistic, additive, and antagonistic effects of food mixtures on total antioxidant capacities. Journal of agricultural and food chemistry. 2011;59:960-8.

236. Chou TC. Theoretical basis, experimental design, and computerized simulation of synergism and antagonism in drug combination studies. Pharmacological reviews. 2006;58:621-81.

237. Chou TC, Talalay P. Quantitative analysis of dose-effect relationships: the combined effects of multiple drugs or enzyme inhibitors. Adv Enzyme Regul. 1984;22:27-55. 238. Steel GG, Peckham MJ. Exploitable mechanisms in combined radiotherapy-chemotherapy: the concept of additivity. Int J Radiat Oncol Biol Phys. 1979;5:85-91.

239. Niu C, Bao H, Tolstykh T, Micolochick Steuer HM, Murakami E, Korba B, et al. Evaluation of the in vitro anti-HBV activity of clevudine in combination with other nucleoside/nucleotide inhibitors. Antivir Ther. 2010;15:401-12.

240. D'Avenia P, Porrello A, Berardo M, Angelo MD, Soddu S, Arcangeli G, et al.

Tp53-gene transfer induces hypersensitivity to low doses of $\mathrm{X}$-rays in glioblastoma cells: a strategy to convert a radio-resistant phenotype into a radiosensitive one. Cancer letters. 2006;231:102-12.

241. Pinto-Leite R, Botelho P, Ribeiro E, Oliveira PA, Santos L. Effect of sirolimus on urinary bladder cancer T24 cell line. Journal of experimental \& clinical cancer research : CR. 2009;28:3.

242. Lee CT, Park KH, Yanagisawa K, Adachi Y, Ohm JE, Nadaf S, et al. Combination therapy with conditionally replicating adenovirus and replication defective adenovirus. 
Cancer Res. 2004;64:6660-5.

243. Lee CT, Lee YJ, Kwon SY, Lee J, Kim KI, Park KH, et al. In vivo imaging of adenovirus transduction and enhanced therapeutic efficacy of combination therapy with conditionally replicating adenovirus and adenovirus-p27. Cancer Res. 2006;66:372-7. 244. Chou TC, Talalay P. Generalized equations for the analysis of inhibitions of Michaelis-Menten and higher-order kinetic systems with two or more mutually exclusive and nonexclusive inhibitors. Eur J Biochem. 1981;115:207-16.

245. Vaha-Koskela MJ, Heikkila JE, Hinkkanen AE. Oncolytic viruses in cancer therapy. Cancer letters. 2007;254:178-216.

246. Yamamoto M, Curiel DT. Current issues and future directions of oncolytic adenoviruses. Mol Ther. 2010;18:243-50.

247. Zeng X, Carlin CR. Host cell autophagy modulates early stages of adenovirus infections in airway epithelial cells. J Virol. 2013;87:2307-19.

248. Randow F, Munz C. Autophagy in the regulation of pathogen replication and adaptive immunity. Trends Immunol. 2012;33:475-87.

249. Saunders RN, Metcalfe MS, Nicholson ML. Rapamycin in transplantation: a review of the evidence. Kidney Int. 2001;59:3-16.

250. Moretti L, Yang ES, Kim KW, Lu B. Autophagy signaling in cancer and its potential as novel target to improve anticancer therapy. Drug Resist Updat. 2007;10:135-43.

251. Sir D, Tian Y, Chen WL, Ann DK, Yen TS, Ou JH. The early autophagic pathway is activated by hepatitis B virus and required for viral DNA replication. Proc Natl Acad Sci U S A. 2010;107:4383-8.

252. Dreux M, Gastaminza P, Wieland SF, Chisari FV. The autophagy machinery is required to initiate hepatitis $\mathrm{C}$ virus replication. Proc Natl Acad Sci U S A. 2009;106:14046-51.

253.Jackson WT, Giddings TH, Jr., Taylor MP, Mulinyawe S, Rabinovitch M, Kopito RR, et al. Subversion of cellular autophagosomal machinery by RNA viruses. PLoS Biol. 2005;3:e156.

254. Faivre S, Kroemer G, Raymond E. Current development of mTOR inhibitors as anticancer agents. Nature reviews Drug discovery. 2006;5:671-88.

255. Sipula IJ, Brown NF, Perdomo G. Rapamycin-mediated inhibition of mammalian target of rapamycin in skeletal muscle cells reduces glucose utilization and increases fatty acid oxidation. Metabolism: clinical and experimental. 2006;55:1637-44.

256. Wei LH, Su H, Hildebrandt IJ, Phelps ME, Czernin J, Weber WA. Changes in tumor metabolism as readout for Mammalian target of rapamycin kinase inhibition by rapamycin in glioblastoma. Clin Cancer Res. 2008;14:3416-26.

257. Edinger AL, Linardic CM, Chiang GG, Thompson CB, Abraham RT. Differential effects of rapamycin on mammalian target of rapamycin signaling functions in mammalian cells. Cancer Res. 2003;63:8451-60.

258. Schieke SM, Phillips D, McCoy JP, Jr., Aponte AM, Shen RF, Balaban RS, et al. The mammalian target of rapamycin (mTOR) pathway regulates mitochondrial oxygen consumption and oxidative capacity. J Biol Chem. 2006;281:27643-52. 259. Cunningham JT, Rodgers JT, Arlow DH, Vazquez F, Mootha VK, Puigserver P. mTOR controls mitochondrial oxidative function through a YY1-PGC-1alpha transcriptional complex. Nature. 2007;450:736-40.

260. Ramanathan A, Schreiber SL. Direct control of mitochondrial function by mTOR. 
Proc Natl Acad Sci U S A. 2009;106:22229-32.

261. He S, Kato K, Jiang J, Wahl DR, Mineishi S, Fisher EM, et al. Characterization of the metabolic phenotype of rapamycin-treated CD8+ T cells with augmented ability to generate long-lasting memory cells. PLoS One. 2011;6:e20107.

262. Fang Y, Westbrook R, Hill C, Boparai RK, Arum O, Spong A, et al. Duration of rapamycin treatment has differential effects on metabolism in mice. Cell metabolism. 2013;17:456-62.

263. Bardell D. Glucose uptake and lactic acid production of adenovirus type 5-infected HEp-2 cells cultured under exponential growth and stationary phase conditions.

Microbios. 1977;20:139-44.

264. Yu Y, Clippinger AJ, Alwine JC. Viral effects on metabolism: changes in glucose and glutamine utilization during human cytomegalovirus infection. Trends Microbiol. 2011;19:360-7.

265. Munger J, Bajad SU, Coller HA, Shenk T, Rabinowitz JD. Dynamics of the cellular metabolome during human cytomegalovirus infection. PLoS pathogens. 2006;2:e132. 266. Sehgal SN. Rapamune (RAPA, rapamycin, sirolimus): mechanism of action immunosuppressive effect results from blockade of signal transduction and inhibition of cell cycle progression. Clin Biochem. 1998;31:335-40.

267. Grewe M, Gansauge F, Schmid RM, Adler G, Seufferlein T. Regulation of cell growth and cyclin D1 expression by the constitutively active FRAP-p70s6K pathway in human pancreatic cancer cells. Cancer Res. 1999;59:3581-7.

268. Gaben AM, Saucier C, Bedin M, Barbu V, Mester J. Rapamycin inhibits cdk4 activation, p21(WAF1/CIP1) expression and G1-phase progression in transformed mouse fibroblasts. International Journal of Cancer. 2004;108:200-6.

269. Kawamata S, Sakaida H, Hori T, Maeda M, Uchiyama T. The upregulation of p27Kip1 by rapamycin results in G1 arrest in exponentially growing T-cell lines. Blood. 1998;91:561-9.

270. Madeo F, Eisenberg T, Buttner S, Ruckenstuhl C, Kroemer G. Spermidine: a novel autophagy inducer and longevity elixir. Autophagy. 2010;6:160-2.

271. Wong VK, Li T, Law BY, Ma ED, Yip NC, Michelangeli F, et al. Saikosaponin-d, a novel SERCA inhibitor, induces autophagic cell death in apoptosis-defective cells. Cell Death Dis. 2013;4:e720.

272. Chu C, Zhang X, Ma W, Li L, Wang W, Shang L, et al. Induction of autophagy by a novel small molecule improves abeta pathology and ameliorates cognitive deficits. PLoS One. 2013;8:e65367. 


\section{APPENDIX}

List of Abbreviations

$\begin{array}{ll}\text { 3-MA } & \text { 3-methyladenine } \\ \text { Ad } & \text { adenovirus } \\ \text { Adwt } & \text { wild-type adenovirus } \\ \text { ATG } & \text { autophagy-related protein } \\ \text { BCL-2 } & \text { B-cell lymphoma 2 } \\ \text { CPE } & \text { cytopathic effect } \\ \text { CDC25 } & \text { cell division cycle 25 } \\ \text { CDK2 } & \text { cyclin-dependent kinase 2 } \\ \text { CHO } & \text { Chinese hamster ovary } \\ \text { CAR } & \text { coxsackievirus and adenovirus receptor } \\ \text { cyclin EL } & \text { large form of cyclin E } \\ \text { CLS } & \text { centrosomal localization signal } \\ \text { CTLs } & \text { cytotoxic T lymphocytes } \\ \text { Cl } & \text { combination index } \\ \text { DNA } & \text { deoxyribonucleic acid } \\ \text { DMSO } & \text { dimethyl sulfoxide } \\ \text { EC } & \text { effective concentration } \\ \text { FBS } & \text { fetal bovine serum }\end{array}$

$\mathrm{Fa}-\mathrm{Cl} \quad$ fraction of virus affected combination index 


\begin{tabular}{|c|c|}
\hline GFP & green fluorescent protein \\
\hline hTERT & human telomerase reverse transcriptase \\
\hline HS-GAGs & heparan sulphate glycosaminoglycans \\
\hline IP & immunoprecipitation \\
\hline IFU & infectious unit \\
\hline $\lg G$ & immunoglobulin G \\
\hline I.O.D & integrated optical density \\
\hline ITR & inverted terminal repeat \\
\hline LC3 & microtubule-associated protein 1 light chanin 3 \\
\hline MDM2 & mouse double minute 2 homolog \\
\hline mRNA & messenger ribonucleic acid \\
\hline MCM & minichromosome maintenance protein \\
\hline MLP & major late promoter \\
\hline mTOR & mammalian target of rapamycin \\
\hline $\mathrm{MOI}$ & multiplicity of infection \\
\hline MEM-Alpha & minimal essential medium Alpha \\
\hline ORF & open reading frame \\
\hline OD & optical density \\
\hline PBS & phosphate buffered saline \\
\hline $\mathrm{Rb}$ & retinoblastoma gene \\
\hline $\mathrm{pRb}$ & retinoblastoma protein \\
\hline phospho-pRb & phosphorylated pRb \\
\hline PCNA & proliferating cell nuclear antigen \\
\hline
\end{tabular}


p.i. post infection

PSA prostate-specific antigen

Ros roscovitine

siRNA small interfering ribonucleic acid

S.D. standard deviation

SDS Sodium dodecyl sulfate 


\title{
CURRICULUM VITAE
}

\section{PEI-HSIN CHENG}

\author{
ADDRESS: $\quad 627$ S. Preston St. Apt. 5K \\ Louisville, KY 40202 \\ DOB: $\quad$ June 19 1983; Kaohsiung City, Taiwan
}

\section{EDUCATION}

2008-Present

Ph.D., Pharmacology and Toxicology

University of Louisville, KY, USA

2005-2007

M.S., Biological Sciences

National Sun Yat-Sen University, Kaohsiung, Taiwan

2001-2005

B.S., Biological Sciences

National Sun Yat-Sen University, Kaohsiung, Taiwan

\section{RESEARCH EXPERIENCE}

2008 - Present

Ph.D. research, Department of Pharmacology and Toxicology, University of Louisville

Supervisor: Kelly M. McMasters, M.D., Ph.D. and H Sam Zhou, Ph.D.

$2007-2008$

Research Assistant, Department of Medical Research and Pediatrics, Chang Gung Memorial Hospital-Kaohsiung Medical Center, Kaohsiung, Taiwan

Principle Investigator: Lin Wang, M.D.

$2005-2007$

M.S. research, Department of Biological Sciences, National Sun Yat-Sen University, Kaohsiung, Taiwan

Supervisor: Shiping He, Ph.D. 


\section{HONORS AND AWARDS}

2010 - Present

Financial Aid Award from Chair Surgery, Department of Surgery, University of

Louisville, KY, USA.

$2008-2010$

Doctoral Fellowship, Integrated Program in Biomedical Science (IPIBS),

University of Louisville, KY, USA.

$2008-2010$

K. C. Huang Scholarship, Department of Pharmacology and Toxicology,

University of Louisville, KY, USA.

$2006-2007$

Graduate Teaching Assistantship, Experimental courses of Plant Physiology and Genetics, Department of Biological Sciences, National Sun Yat-Sen University, Kaohsiung, Taiwan.

$2006-2007$

Graduate Research Assistantship, Laboratory of Molecular Genetics, Department of Biological Sciences, National Sun Yat-Sen University, Kaohsiung, Taiwan.

2005

Graduate with Honor, Department of Biological Sciences, National Sun Yat-Sen University, Kaohsiung, Taiwan.

\section{PROFESSIONAL SOCIETIES}

2012 - Present

American Society for Pharmacology and Experimental Therapeutics

2009 - Present

Golden Key International Honors Society

2006 - Present

Chinese Society of Cell and Molecular Biology

\section{PUBLICATIONS}

Pei-Hsin Cheng, Serena Lian, Robin Zhao, Xiao-Mei Rao, Kelly M. McMasters, and $\mathrm{H}$. Sam Zhou. Combination of autophagy inducer rapamycin and oncolytic adenovirus improves antitumor effect in cancer cells.

Virology Journal 10(1):293. doi:10.1186/1743-422X-10-293. 2013 Sep 23. 
Pei-Hsin Cheng, Xiao-Mei Rao, Kelly M. McMasters, and H. Sam Zhou. Molecular basis for viral selective replication in cancer cells: activation of CDK2 by adenovirus-induced cyclin $\mathrm{E}$.

PLoS ONE 8(2):e57340. doi:10.1371/journal.pone.0057340. Epub 2013 Feb 20.

\section{MANUSCRIPTS IN PREPARATION}

Pei-Hsin Cheng, Xiao-Mei Rao, Kelly M. McMasters, and H. Sam Zhou. Properties of oncolytic replication of adenoviruses in murine and human lung cancer cells. (To be submitted to Cancer Letters)

Pei-Hsin Cheng, Xiao-Mei Rao, XiaoXian Duan, Xiao-Feng Li, Michael E. Egger, Kelly M. McMasters, and H. Sam Zhou. Oncolytic adenovirus targeting cyclin E overexpression in cancer inhibits tumor development in vivo. (To be submitted to Molecular Cancer)

Pei-Hsin Cheng, Stephen L. Wechman, Kelly M. McMasters, and H. Sam Zhou. Molecular mechanisms of oncolytic therapy with adenoviruses. (Review article, to be submitted to Molecular Therapy)

Stephen L. Wechman, Pei-Hsin Cheng, Kelly M. McMasters, and H. Sam Zhou. Insights into optimized oncolytic adenovirus design. (Review article)

\section{ABSTRACTS SUBMITTED TO REGIONAL AND NATIONAL MEETINGS}

Pei-Hsin Cheng, Xiao-Mei Rao, Kelly M. McMasters, and H. Sam Zhou. Oncolytic adenoviral therapy enhanced by targeting cyclin $\mathrm{E}$ overexpression and inducing autophagy. Experimental Biology, Boston, MA, 2013.

Pei-Hsin Cheng, Xiao-Mei Rao, Kelly M. McMasters, and H. Sam Zhou. Oncolytic virotherapy of lung cancer in vitro with a novel cyclin E promoter-controlled oncolytic adenovirus. Research Louisville, Louisville, KY, 2012.

Pei-Hsin Cheng, Xiao-Mei Rao, Kelly M. McMasters, and H. Sam Zhou. Human Adenoviruses replication in human and mouse lung cancer cells. 10th James Graham Brown Cancer Center Annual Retreat, Louisville, KY, 2011.

Pei-Hsin Cheng, Xiao-Mei Rao, Kelly M. McMasters, and H. Sam Zhou. Oncolytic Adenovirus Replication Depends on Cyclin E Activating CDK2. Research Louisville, Louisville, KY, 2010. 
Pei-Hsin Cheng, Ya-Ling Lin, and Shiping He. Expression and characterization of truncated HGF in human breast cancer cell. 22th Joint Annual Conference of Biomedical Sciences, Taipei, Taiwan, 2007.

\section{TECHNICAL SKILLS}

Molecular biology: general molecular biology procedures including PCR, molecular cloning, Southern and Western blots, immunoprecipitation, recombinant protein expression, purification and characterization.

Cellular biology: cell culture and general assays, viral and non-viral gene delivery systems, cell cloning and staining.

In vivo studies: drug delivery techniques including intravenous, intraperitoneal and intratumoral injections, developing subcutaneous and orthotopic mouse models of lung cancer, monitoring tumor growth, immunoassays, hematoxylin-eosin staining and immunohistochemistry.

Computer literacy: Microsoft Office (Excel, PowerPoint and Word), citation management software (EndNote), image analysis softwares (Gel-Pro analyzer and ImageJ), analyzer of combined drug effects (CalcuSyn). 